\title{
Biodegradation of Plasticizers by Rhodotorula rubra
}

\author{
by \\ James Gartshore \\ Department of Chemical Engineering \\ McGill University, Montreal
}

November, 2001

A thesis submitted to the Faculty of Graduate Studies and Research in partial fulfillment of the requirements of the degree of Master of Engineering. 
National Library of Canada

Acquisitions and Bibliographic Services

305 Wellington Streot Oraw ON KIA ONA Cansode

\section{Bibliotheque nationale}

du Canada

Acquisitions et

services bibliographiques

395 ne Wallingtion

Orime ON KIA ONA

Canade

rac in noov nom

The author has granted a nonexchusive licence allowing the National Library of Canada to reproduce, loan, distribute or sell copies of this thesis in microform, paper or electronic formats.

The author retains ownership of the copyright in this thesis. Neither the thesis nor substantial extracts from it may be printed or otherwise reproduced without the author's permission.
L'auteur a accordé une licence non exclusive permettant à la Bibliothèque nationale du Canada de reproduire, prêter, distribuer ou vendre des copies de cette thèse sous la forme de microfiche/film, de reproduction sur papier ou sur format électronique.

L'auteur conserve la propriété du droit d'auteur qui protège cette thèse. Ni la thèse ni des extraits substantiels de celle-ci ne doivent être imprimés ou autrement reproduits sans son autorisation. 


\begin{abstract}
The degradation of plasticizers by the yeast Rhodotorula rubra J-96-1 (ATCC 9449) was studied in the presence of a water-soluble substrate (glucose). The plasticizers studied included bis 2-ethylhexyl adipate (B(EH)A), dioctyl phthalate (DOP) and terephthalate (DOTP), which are in widespread use. In addition, the degradation of two less common plasticizers, di-propylene glycol dibenzoate $(\mathrm{D}(\mathrm{PG}) \mathrm{DB})$ and di-ethylene glycol dibenzoate (D(EG)DB), were studied. It has been proposed that the latter plasticizers be used as alternatives to the commonly used plasticizers, which have been associated with negative environmental impacts.

The degradation of $\mathrm{D}(\mathrm{PG}) \mathrm{DB}$ or $\mathrm{D}(\mathrm{EG}) \mathrm{DB}$ led to a significant increase in solution toxicity. This increase in toxicity was associated with the production of metabolites resulting from the incomplete breakdown of the original plasticizers. The metabolites responsible for the acute toxicity in the $\mathrm{D}(\mathrm{PG}) \mathrm{DB}$ system were identified as isomers of di-propylene glycol monobenzoate. A mechanism for the formation of this metabolite was proposed. Although the metabolite observed when D(EG)DB was being degraded was not isolated, it was tentatively identified as di-ethylene glycol monobenzoate by analogy to the $\mathrm{D}(\mathrm{PG}) \mathrm{DB}$ system. This same metabolite was observed when D(EG)DB was degraded by the fungus, Aspergillus niger ATCC 9642-U.
\end{abstract}

In contrast, there were no observable metabolites nor increases in toxicity in the media during the degradation of $\mathrm{B}(\mathrm{EH}) \mathrm{A}, \mathrm{DOP}$, or DOTP by $R$. rubra. These observations also differ from those of earlier work in which it was reported that the degradation of all three of these plasticizers by bacteria resulted in the production of toxic metabolites. 
Collectively, these results do not support the use of $D(P G) D B$ and $D(E G) D B$ as environmentally safe alternatives to $\mathrm{B}(\mathrm{EH}) \mathrm{A}$, DOP or DOTP. 


\section{RÉSUMÉ}

La dégradation de plastifiants par la levure Rhodotorula rubra J-96-1 (ATCC 9449) a été étudiée en présence d'un substrat soluble dans l'eau (glucose). Des plastifiants utilisés couramment, incluant le bis 2-éthylhexyl adipate $(\mathrm{B}(\mathrm{EH}) \mathrm{A})$ et le dioctyl phtalate (DOP) et téréphtalate (DOTP) ont été étudiés. De plus, la dégradation de deux plastifiants moins communs, le di-propylène glycol dibenzoate $(\mathrm{D}(\mathrm{PG}) \mathrm{DB})$ et le diéthylène glycol dibenzoate $(\mathrm{D}(\mathrm{EG}) \mathrm{DB})$, a été étudiée. Il a déja été suggéré de remplacer les plastifiants couramment utilisés, associés à des impacts négatifs sur l'environnement, par ces derniers plastifiants.

La dégradation de $\mathrm{D}(\mathrm{PG}) \mathrm{DB}$ ou $\mathrm{D}(\mathrm{EG}) \mathrm{DB}$ a provoqué une augmentation significative de la toxicité de la solution. Cette augmentation de toxicité a été associée à la production de métabolites résultant de la dégradation incomplète des plastifiants initiaux. Les isomères de di-propylène glycol monobenzoate ont été identifiés comme étant les métabolites responsables de 1'augmentation de toxicité du système D(PG)DB. Un mécanisme de formation du métabolite a été proposé. Dans le cas du système $\mathrm{D}(\mathrm{EG}) \mathrm{DB}$, le métabolite n'ayant pas été isolé, mais par analogie avec le système $\mathrm{D}(\mathrm{PG}) \mathrm{DB}$ il est présumé que le métabolite est le di-éthylène glycol monobenzoate. $\mathrm{Ce}$ même métabolite a été observé lorsque le $\mathrm{D}(\mathrm{EG}) \mathrm{DB}$ était dégradé par le champignon Aspergillus niger ATCC 9642-U.

A l'opposé, aucun métabolite ou augmentation de toxicité n’a été observé lors de la dégradation de $\mathrm{B}(\mathrm{HE}) \mathrm{A}, \mathrm{DOP}$, ou DOTP par $R$. rubra. Ces observations diffèrent de celles d'une étude précédente où la dégradation de ces trois plastifiants par des bactéries résultait en une production de métabolites toxiques. 
Globalement, ces résultats ne supportent pas 1'utilisation $\mathrm{D}(\mathrm{PG}) \mathrm{DB}$ et $\mathrm{D}(\mathrm{EG}) \mathrm{DB}$ comme alternative aux B(EH)A, DOP ou DOTP qui soit sécuritaire pour l'environnement. 


\section{ACKNOWLEDGEMENTS}

First and foremost I must thank Dr. Cooper for giving me the opportunity to pursue this graduate degree and for all the sound advice, patience, and guidance throughout the course of this project. Your friendship and infinite wisdom (and those deep pockets at T.H.!) have helped make these last two years incredible. I would also

like to thank Dr. Nicell for all of his help with this project (especially in getting the thesis done!).

To Dave Cavalero, Sandro Nalli and Joe Marin in the Falcon Research Group: JESUS! I've never "worked" with a finer group of individuals in my life. I'll never forget the laughs, good times and significant number of hangovers on Saturday morning. If we had a video camera or tape recorder in London and Halifax, we could be millionaires right now (that or prosecuted...). I'd like to thank the following people for their support as well: Carl, Annie, the Heaths, Esther, Rob Pinchuck, Yin Choi, John, Wayne and Fabe. And also to the summer students: Robbie, Dom, J-P, Eric, Owen, Laura, and Shaddick for his debaucherous contributions to the road trips and for getting me hooked on golf. If I've forgotten anyone, I apologize but you know who you are!

I would also like to thank the entire department of Chemical Engineering for their help and guidance over the last two years. Special thanks to Dr. Munz, Ed, Lou, Frank, and all the Chem. Eng. secretaries.

Finally, thank you to both of my families for your love and support and to my future wife Julia Esteves for your love, dedication, patience and for understanding that it's all right to get out of the lab and go out with the boys every once and a while (thanks Kyle, Andy, Pete and the South Shore group)! 


\section{TABLE OF CONTENTS}

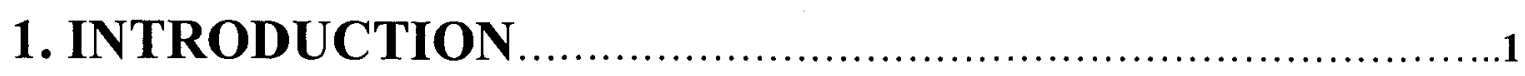

1.1. Plasticizers and Their Role in Industry................................

1.2. Environmental Impact of Plasticizers...............................

1.2.1. Leaching and Migration of Plasticizers............................5

1.2.2. Biological Interactions With Plasticizers..........................6

1.2.3. Bioremediation as a Solution.................................

1.2.4. Interactions of Yeasts and Fungi with Hydrocarbons................ 8

1.3. Objectives................................................................. 10

2. MATERIALS AND METHODS ................................12

2.1. Microorganisms................................................... 12

2.2. Shake Flask Experiments........................................ 14

2.3. Batch Reactor Experiments........................................ 15

2.4. Biomass Determination.............................................. 17

2.4.1. Dry Weight Measurement......................................17

2.4.2. Protein Concentration of Biomass.................................17

2.5. Analysis of Media Components......................................19

2.5.1. Plasticizers and Metabolites.......................................19

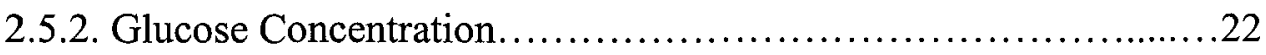

2.6. Toxicity Measurements..........................................24

2.6.1. Microtox Assay .................................................24

2.6.2. Toxicity of Phases from Extraction Procedure......................25

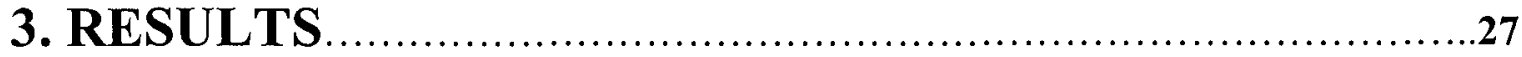

3.1 Preliminary Growth Studies.....................................27

3.2 Batch Fermentations..............................................29

3.2.1. Plasticizer Sampling Error.....................................29

3.2.2. Use of Measurement of Total Protein for Biomass.................31

3.2.3. Fermentations................................................... 33

3.2.4. Characterization of Metabolites...............................47

3.3. Toxicity of Pure Compounds.....................................58 
4.1. Biodegradation of Plasticizers by Eukaryotes........................60

4.1.1. Preliminary Screening of Plasticizers..........................60

4.1.2. Degradation of Dibenzoate....................................61

4.1.3. Degradation of B(EH)A, DOP, and DOTP Plasticizers ............65

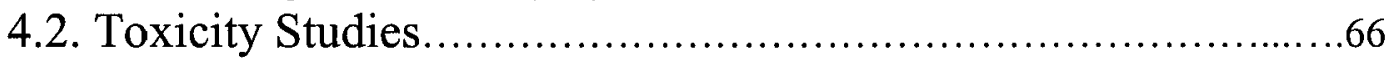

4.3. Potential Impact.................................................... 70

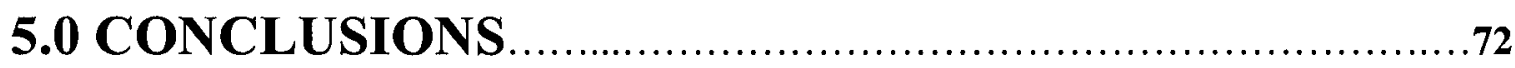

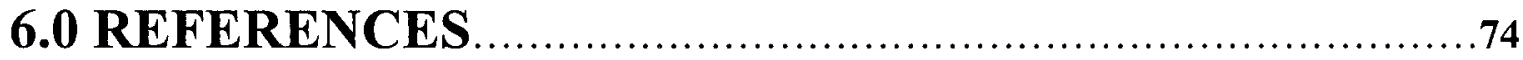

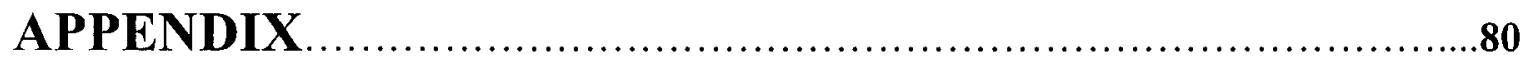

A.1. Synthesis Reactions for Di-Propylene Glycol Monobenzoate....81

A.2. Isolation of Di-Propylene Glycol Monobenzoate..................83 


\section{LIST OF TABLES}

Table 2-1. Microorganisms used........................................... 12

Table 2-2. Composition of modified minimum mineral salt solution.................13

Table 2-3. Gas chromatography operation conditions...........................20

Table 2-4. Gas chromatography-mass spectrometry operation conditions..............20

Table 3-1. Degradation of plasticizers by eukaryotes in media containing.............28 plasticizer and a co-substrate.

Table 3-2. Gas chromatograph-mass spectrophotometer (GC-MS) data for............49 metabolites, plasticizers and other data.

Table 3-3. Toxicity of compounds in MMSM....................................59

Table A-1. $R_{F}$ values for bands separated from the crude product of the $\ldots \ldots \ldots \ldots \ldots 84$ trans-esterification reaction.

Table A-2. $\mathrm{R}_{\mathrm{F}}$ values for bands separated from the crude product of the ..............84 ester synthesis reaction. 


\section{LIST OF FIGURES}

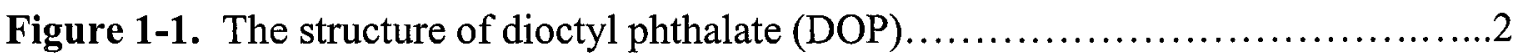

Figure 1-2. The structure of dioctyl terephthalate (DOTP) $\ldots \ldots \ldots \ldots \ldots \ldots \ldots \ldots \ldots . \ldots \ldots$

Figure 1-3. The structure of bis (2-ethylhexyl) adipate $(\mathrm{B}(\mathrm{EH}) \mathrm{A}) \ldots \ldots \ldots \ldots \ldots \ldots \ldots \ldots . . . \ldots \ldots$

Figure 1-4. The structure of di-propylene glycol dibenzoate (D(PG)DB) .............4

Figure 1-5. The structure of di-ethylene glycol dibenzoate (D(PG)DB) ..............4

Figure 2-1. Schematic of the batch reactor.................................. 16

Figure 2-2. Calibration curve for protein concentration $(\diamond) \ldots \ldots \ldots \ldots \ldots \ldots \ldots \ldots \ldots$

Figure 2-3. Calibration curve for di-ethylene glycol dibenzoate (ㅌ) $) \ldots \ldots \ldots \ldots \ldots \ldots . \ldots 21$

Figure 2-4. Calibration curve for the metabolite (․․ from di-propylene...............21 glycol dibenzoate.

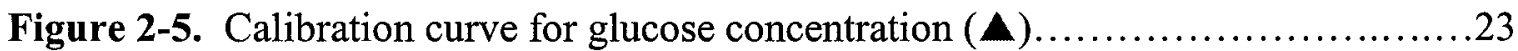

Figure 3-1. Growth of $R$. rubra expressed as protein (A), on a medium..............30 containing di-propylene glycol dibenzoate ( $\mathbf{E})$ and glucose.

Figure 3-2. Dry weight (O) and protein (A) for the growth of $R$. rubra in a medium containing di-propylene glycol dibenzoate and glucose.

Figure 3-3. Growth of $R$. rubra expressed as protein $(\boldsymbol{\Delta})$ and Toxicity $(\bullet)$ .34 on a medium containing solely glucose.

Figure 3-4. For a system using $R$. rubra: (a) protein (A) and glucose. .35 concentration ( $)$ (b) concentration of di-propylene glycol dibenzoate (1) and a pattern of metabolites ( The initial amount of plasticizer added to the reactor is also shown ( $\square$ ).

Figure 3-5. The concentration of a pattern of metabolites (c) and. toxicity $(\bullet)$ for the system represented in Figure 3-4 (a), (b).

Figure 3-6. The concentration of a pattern of metabolites ( $)$ and 37 toxicity $(\checkmark)$ from the repeat of the experiment represented in Figure 3-4 (a), (b). 
Figure 3-7. For a system using $R$. rubra: (a) protein (A) and glucose concentration ( $)$ (b) concentration of di-ethylene glycol dibenzoate (삐) and a metabolite ( ). The initial amount of plasticizer added to the reactor is also shown ( $\square$ ).

Figure 3-8. GC area ratio of a metabolite (수 ) and toxicity ( $\bullet$ ) for the system represented in Figure 3-7 (a), (b).

Figure 3-9. For a system using $A$. niger: (a) protein ( $\mathbf{A})$ and glucose concentration (b) (b) concentration of di-ethylene glycol dibenzoate ( $)$ and a metabolite ( $)$. The initial amount of plasticizer added to the reactor is also shown ( $\square$ ).

Figure 3-10. GC area ratio of a metabolite $(\bullet)$ and the toxicity $(\diamond)$ .42 for the system represented in Figure 3-9 (a), (b).

Figure 3-11. For a system using $R$. rubra: (a) protein ( $\mathbf{\Delta})$ and glucose. .44 concentration (-) (b) concentration of bis (2-ethylhexyl) adipate ( $\mathbf{E})$ and toxicity $(\bullet)$. The initial amount of plasticizer added to the reactor is also shown $(\square)$.

Figure 3-12. For a system using R. rubra: (a) protein (A) and glucose. .45 concentration (b) (b) concentration of dioctyl phthalate ( $\boldsymbol{( a )}$ and toxicity $(\bullet)$. The initial amount of plasticizer added to the reactor is also shown ( $\square)$.

Figure 3-13. For a system using $R$. rubra: (a) protein (A) and glucose. .46 concentration ( $)$ (b) concentration of dioctyl terephthalate $(\mathbf{W})$ and toxicity $(\checkmark)$. The initial amount of plasticizer added to the reactor is also shown ( $\square)$.

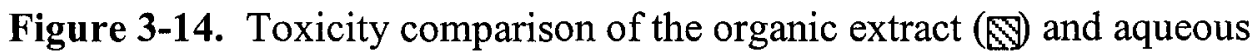
.48 raffinate $(\square)$ obtained from original samples $:)$ taken from the fermentation of D(PG)DB.

Figure 3-15. Gas chromatograph of a di-propylene glycol dibenzoate...............50 reactor sample.

Figure 3-16. Gas chromatograph of a di-ethylene glycol dibenzoate................50 reactor sample.

Figure 3-17. Gas chromatograph of the crude product obtained from .52 a trans esterification reaction. 
Figure 3-18. Gas chromatograph of the crude product obtained from..................52 an ester synthesis reaction.

Figure 3-19. Isomer configurations of di-propylene glycol monobenzoate..............53

Figure 3-20. Mass spectrum fragmentation pattern of the pattern of..................54 metabolites observed in a di-propylene glycol dibenzoate reactor sample.

Figure 3-21. Mass spectrum fragmentation pattern of the family of peaks............55 observed in the crude product from a trans esterification reaction.

Figure 3-22. Mass spectrum fragmentation pattern of the family of peaks.............55 observed in the crude product from an ester synthesis reaction.

Figure 3-23. Mass spectrum fragmentation pattern of the single peak...............57 observed in a di-ethylene glycol dibenzoate reactor sample.

Figure 4-1. Proposed mechanism for the production of di-propylene glycol...........63 monobenzoate from the degradation of $\mathrm{D}(\mathrm{PG}) \mathrm{DB}$. 


\section{INTRODUCTION}

\subsection{Plasticizers and Their Role in Industry}

Plastics have many different practical applications in industry including their use in the manufacture of automobiles, household products, toys, medical supplies, wrappings and building materials for the construction industry. The ease of production and formation of plastics make them ideal materials to work with.

All plastics are polymeric materials that frequently contain a number of different chemical additives to aid in their processing. An important group of these chemicals are called plasticizers. These are lipophilic compounds that are relatively small compared to the molecular weights of the long-chain hydrocarbon polymers, which comprise the backbone of the plastics. Plasticizers often contain aromatic groups as well.

The most commonly used plastic in the above-mentioned industries is polymerized vinyl chloride (PVC), which was first introduced in $1931^{21}$. Many different types of plasticizers are added to this and other polymers to enhance certain desired qualities such as workability and flexibility ${ }^{54}$. Dioctyl phthalate (DOP) is the most common plasticizer added to PVC and is shown in Figure 1-1. DOP is classified as a monomeric plasticizer, which is a grouping of plasticizers that account for close to $80 \%$ of the plasticizer market ${ }^{17}$. DOP was introduced in $1933^{15}$ and now accounts for $50 \%$ of all phthalate plasticizers used in the production of plastics ${ }^{38}$. An estimated 500 million kilograms of DOP is used each year in industry ${ }^{60}$.

Another commonly used phthalate plasticizer is dioctyl terephthalate (DOTP). As Figure 1-2 demonstrates, DOTP is an isomer of DOP. This plasticizer is used as a less toxic alternative for dioctyl phthalate since there is a possibility of producing the 


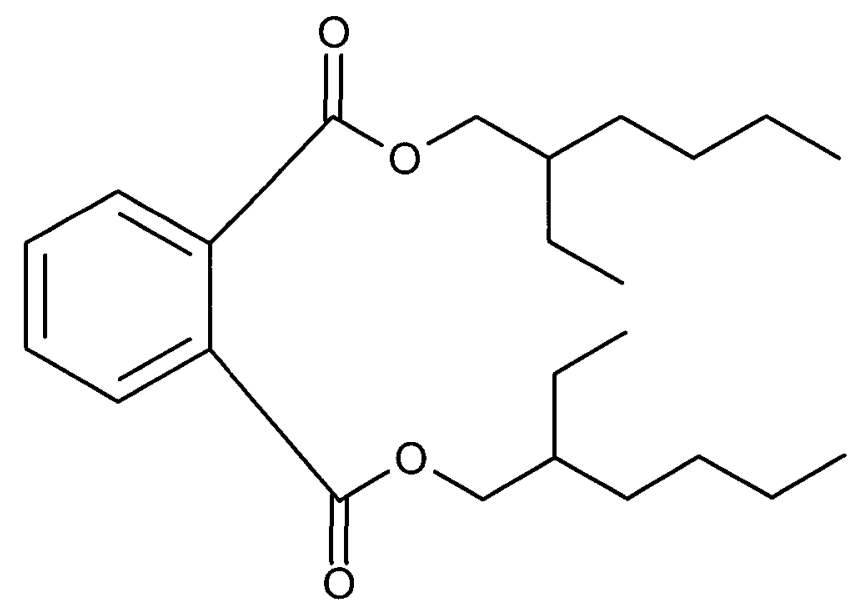

Figure 1-1. The structure of dioctyl phthalate (DOP).

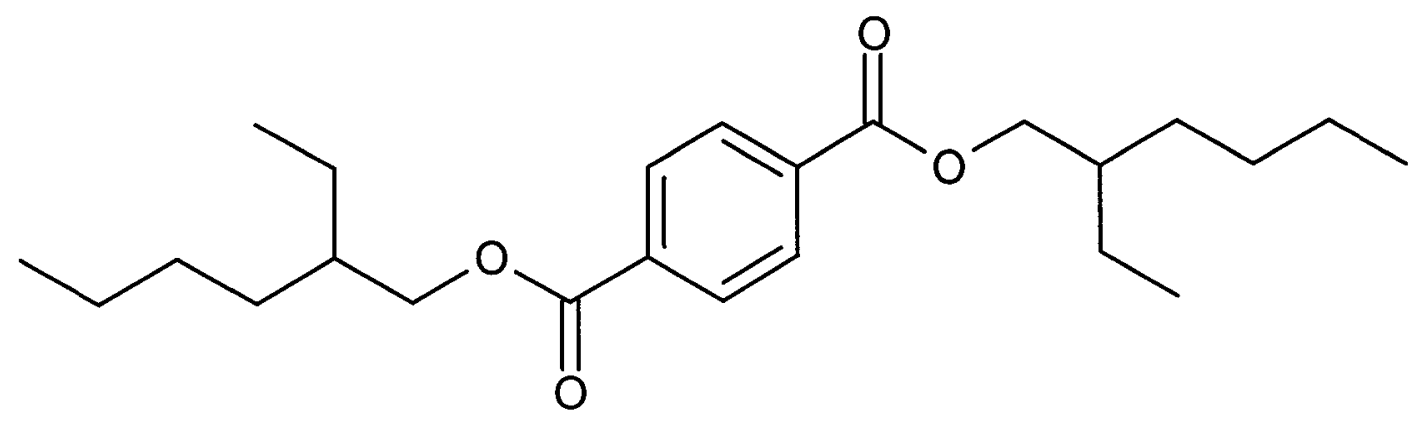

Figure 1-2. The structure of dioctyl terephthalate (DOTP). 
monoester (mono-ethylhexyl phthalate) from the incomplete breakdown of $\mathrm{DOP}^{8,35}$. This metabolite has been causally linked to cancer in higher organisms ${ }^{1}$.

Bis (2-ethylhexyl) adipate $(\mathrm{B}(\mathrm{EH}) \mathrm{A})$ is classified as a monomeric plasticizer and accounts for approximately $5 \%$ of the plasticizer market ${ }^{17}$. The structure of this compound is shown in Figure 1-3. B(EH)A offers the advantage of introducing low temperature properties and flexibility to PVC films ${ }^{17}$. Thus, it is used in the wrappings for refrigerated food products ${ }^{21}$.

Di-propylene glycol dibenzoate $(\mathrm{D}(\mathrm{PG}) \mathrm{DB})$ and di-ethylene glycol dibenzoate $(\mathrm{D}(\mathrm{EG}) \mathrm{DB})$ are classified as benzoic acid ester plasticizers ${ }^{17}$. These two diesters (Figures $1-4,1-5)$ are more commonly referred to as "Benzoflex" in industry and represent examples of less commonly used plasticizers. They are sometimes added to other plasticizers to obtain specific characteristics such as decreased volatilization and fire resistance and are generally restricted to their use in resilient vinyl flooring ${ }^{10,50}$, thermoplastic resins, and hot-melt PVC adhesives ${ }^{17}$.

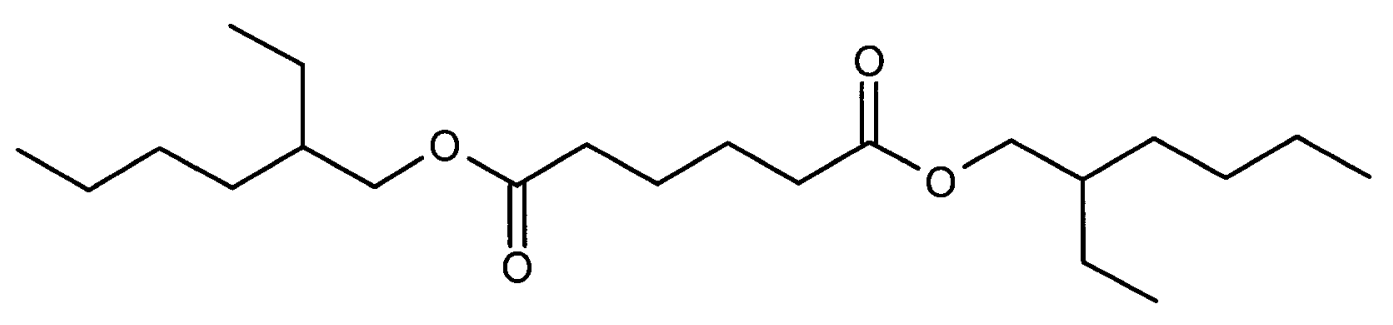

Figure 1-3. The structure of bis (2-ethylhexyl) adipate $(\mathrm{B}(\mathrm{EH}) \mathrm{A})$. 


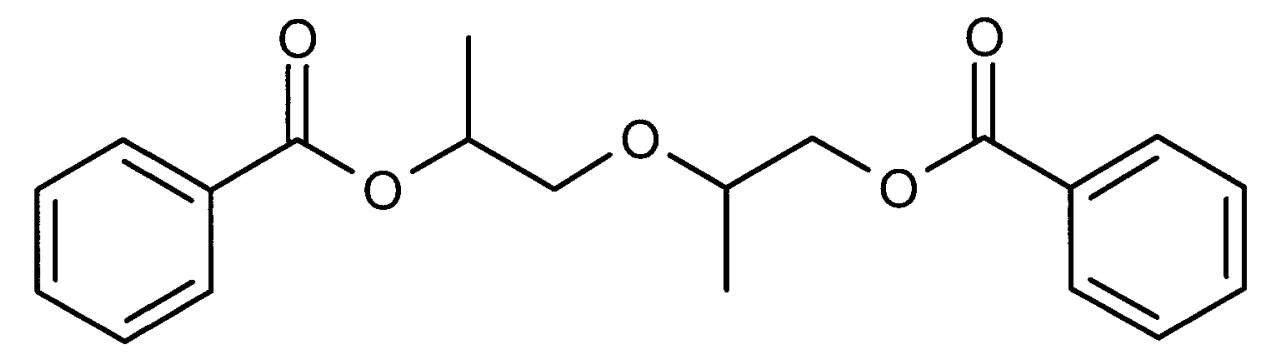

Figure 1-4. The structure of di-propylene glycol dibenzoate (D(PG)DB).

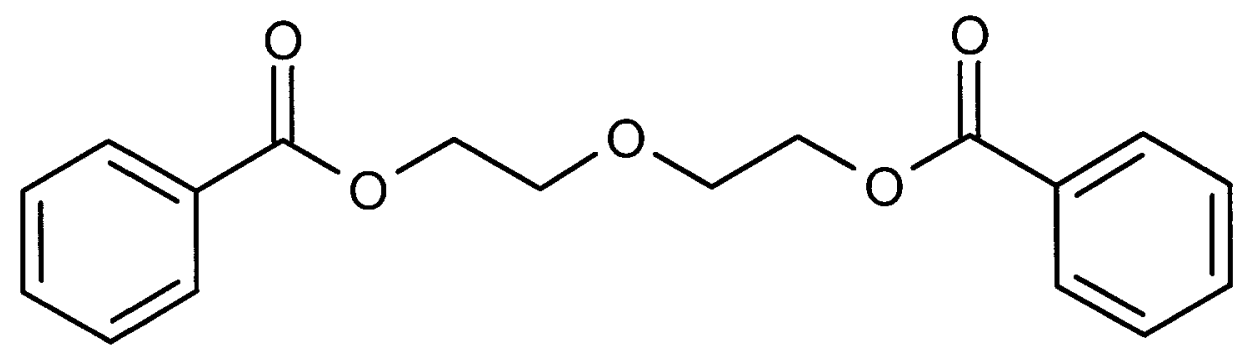

Figure 1-5. The structure of di-ethylene glycol dibenzoate (D(PG)DB). 


\subsection{Environmental Impact of Plasticizers}

\subsubsection{Leaching and Migration of Plasticizers}

An important concern associated with plasticizers is the problem of their leaching and migration from plastic materials and into the environment. These problems are significant because the solid matrix of plastic materials usually contain high concentrations of various plasticizers. For example, PVC can contain up to $40 \%$ by weight plasticizers ${ }^{21}$. Given that plasticizers are small compounds relative to the polymers, once they leach out of the plastic they are free to interact in the environment. Although these plasticizers have very low solubility in the aqueous phase, the presence of surfactants and emulsifiers has been shown to increase their entrainment in this phase $\mathrm{e}^{20}$. Many factors can affect the leaching and migration of different plasticizers ${ }^{14}$. For example, the rate of diffusion of di-ethylene glycol dibenzoate from PVC was found to increase rapidly with increasing temperature ${ }^{43}$. The type of substance in contact with a plastic can also influence the rate of migration of plasticizers ${ }^{21}$. High levels of leached plasticizers from blood bags made of PVC were found to accumulate in the lungs, liver and spleens of blood transfusion patients ${ }^{18}$. Work done with bis (2-ethylhexyl) adipate in contact with different foods has shown how readily this plasticizer leaches out of plastic film wrappings ${ }^{13}$. Similar work done with other types of plasticizers has shown that this is a particular concern for fatty foods such as dairy products ${ }^{56}$. Di-propylene and diethylene glycol dibenzoate have been shown to migrate from microwave susceptible packaging when food-simulating liquids and food such as french fries were cooked in contact with the film ${ }^{9}$. It has been estimated that an average of $8.2 \mathrm{mg} /$ person of plasticizer is ingested each day because of this migration into food products ${ }^{37}$. Other 
work on plasticizer mobility ${ }^{24,33,46}$ have demonstrated that a significant quantity of plasticizers can leach into the environment. Traces of plasticizers have been found in lakes, riverbeds and groundwater near many industrial areas ${ }^{59}$.

\subsubsection{Biological Interactions With Plasticizers}

Since it is nearly impossible to avoid coming into contact with plasticizers in every day life, concerns have recently been raised about the potential impacts that may arise from the exposure of higher organisms to these plasticizers. For example, phthalate esters have recently been implicated as endocrine disruptors ${ }^{23,25,39,41}$. Endocrine disruptors are molecules that mimic different hormones in the body, thus disrupting normal endocrine functions ${ }^{25}$. Low concentrations of dioctyl phthalate (DOP) and dibutyl phthalate (DBP) found in fish near industrialized areas inhibited their normal growth and reproduction ${ }^{58}$. There has also been evidence that DBP causes the early onset of puberty in human females ${ }^{15}$. This recently led to a recall in the United States of cosmetics containing this plasticizer ${ }^{19}$.

In addition, the accumulation of metabolites (2-ethyl hexanoic acid, 2-ethyl hexanol and mono-ethylhexyl phthalate) from the partial breakdown of phthalate and adipate plasticizers has been linked to peroxisome proliferation of liver cells in rats and mice $28,35,42$. The proliferation led to the formation of tumors and the eventual death of the animals. 


\subsubsection{Bioremediation as a Solution}

Given that there are important health implications associated with the phthalate and adipate plasticizers, there is likely to be a large problem if these compounds accumulate in the environment. However, after these plasticizers have leached out of the plastic materials they can come into contact with many different microorganisms that could help remediate this potential problem. Work done with some bacteria has demonstrated that certain phthalate plasticizers can be used as a carbon source and can be completely converted to carbon dioxide ${ }^{16,29}$. Other, more recent work, done by Nalli ${ }^{44}$ using Rhodococcus rhodochrous bacteria has demonstrated that bioremediation of more commonly used plasticizers is not a reliable solution. The degradation of dioctyl phthalate (DOP), dioctyl terephthalate (DOTP), or bis (2-ethylhexyl) adipate (B(EHA)) resulted in the accumulation of metabolites from the incomplete breakdown of the plasticizers. These metabolites (2-ethylhexyl hexanoic acid and 2-ethyl hexanol) were found to be the same ones suspected as being responsible for peroxisome proliferation in rats and mice. Moreover, the metabolites demonstrated acute toxicity, which resulted in a significant increase in the toxicity of the fermentation broths ${ }^{44}$.

The Velsicol Chemical Corporation has published a series of papers that demonstrate the advantages of using dibenzoate plasticizers as opposed to more commonly used plasticizers. Certain blends of dibenzoate plasticizers have demonstrated resistance to fungal growth ${ }^{3}$ and resistance to plasticizer migration ${ }^{55}$. Comparisons of the performance properties with other plasticizers have shown that di-propylene and diethylene glycol dibenzoates $(\mathrm{D}(\mathrm{PG}) \mathrm{DB}$ and $\mathrm{D}(\mathrm{EG}) \mathrm{DB})$ are good alternatives in latex caulks $^{5}$ and in PVC products ${ }^{2,4}$. Lang and Stanhope argue that a blend of dibenzoate 
plasticizers can be used as an alternative for a standard phthalate plasticizer used in children's toys due to its processing ease, final product performance and, more importantly, low toxicity and ease of biodegradation ${ }^{32}$. Other work has also suggested D(EG)DB can completely replace dioctyl phthalate in PVC products as a less toxic plasticizer with a lower production $\operatorname{cost}^{49}$.

It is conceivable that the replacement of more commonly used plasticizers (DOP, DOTP and $\mathrm{B}(\mathrm{EH}) \mathrm{A})$ with dibenzoate plasticizers ( $\mathrm{D}(\mathrm{PG}) \mathrm{DB}$ and $\mathrm{D}(\mathrm{EG}) \mathrm{DB})$ may be a solution to the problems associated with phthalates and adipates. However, the replacement can only be justified as long as the degradation of dibenzoate plasticizers does not lead to the production of toxic metabolites.

\subsubsection{Interactions of Yeasts and Fungi with Hydrocarbons}

Yeasts and fungi have become predominant microorganisms in certain environments that are heavily contaminated by aliphatic and aromatic hydrocarbons ${ }^{52}$. Therefore, it is important to consider their interactions with these contaminants since they are related to plasticizers, which normally contain hydrocarbon chains and aromatic ester groups.

In general, there are a number of differences in the means by which hydrocarbons are degraded by yeasts, fungi and bacteria. Reports on the biodegradation of aromatic hydrocarbons under aerobic conditions have shown that differences exist between the metabolic pathways of biodegradation in yeasts, fungi and bacteria ${ }^{6}$. For example, a study conducted by Walker et al., on the ability of petroleum-degrading yeasts, fungi, and bacteria to degrade a mixed hydrocarbon substrate containing aliphatic and aromatic 
compounds demonstrated that hydrocarbons were less susceptible to degradation by bacteria and yeasts as the carbon chain length increased ${ }^{61}$. Filamentous fungi seemed to be more robust as there was little correlation between chain length and susceptibility of biodegradation. However, another study found that a yeast, Candida tenuis, was able to metabolize a whole range of fuel hydrocarbons while other fungi were more specific and degraded only the aliphatic fraction of the fuels ${ }^{36}$. In all cases, the organisms produced surfactants and emulsifiers at various stages of growth to aid in the degradation of the insoluble hydrocarbons.

Work on the degradation of alkyl aromatic hydrocarbons such as phenylhexadecane and phenylundecane by the yeast Candida guilliermondii resulted in the production of metabolites (phenylacetic acid and phenylpropionic acid) from the incomplete degradation of these compounds ${ }^{57}$. These metabolites were found to inhibit further growth by this organism. The degradation of phenol by another yeast, Rhodotorula rubra, also resulted in the production of metabolites, which were identified using GC-MS analysis ${ }^{26}$. These are examples of interactions that may occur in the environment as a result of the breakdown of a plasticizer with yeasts or fungi.

Some work on the degradation of plasticized polyvinyl systems showed the ability of several yeasts to degrade a variety of commonly used plasticizers, including some aromatic esters that were considered resistant to microbial degradation ${ }^{45}$. Other work selected for and isolated at least 19 different filamentous microorganisms that were able to use dioctyl phthalate (DOP) or dibutyl phthalate (DBP) as the sole carbon source $^{48}$. Most notable among these microorganisms was the yeast Rhodotorula rubra J96-1 and the fungus Aspergillus puniceus J-86-1. At the highest DOP concentration of 
$2 \mathrm{~g} / \mathrm{L}$ in shake flask experiments, $R$. rubra was able to completely break down the original plasticizer in 100 hours whereas $A$. puniceus was able to break down DOP by $15 \%$ in 200 hours $^{47}$. Both yeast and fungi have been shown to produce an extracellular esterase, which would be used to catalyze the initial hydrolysis of the phthalate ester to phthalic acid ${ }^{48}$. From the results of this work it appears the yeast, $R$. rubra, was able to further metabolize this acid whereas the fungus and possibly the other microorganisms in this study were most likely inhibited by the accumulation of this metabolite.

\subsection{Objectives}

The leaching and migration of plasticizers from plastic materials into the environment is of major concern due to the potential problems associated with these compounds. The results of some work on the bioremediation of more commonly used plasticizers (DOP, DOTP, and B(EH)A) with bacteria has been inconclusive.

Furthermore, little is known about the fate of possible alternative or replacement plasticizers such as $\mathrm{D}(\mathrm{PG}) \mathrm{DB}$ and $\mathrm{D}(\mathrm{EG}) \mathrm{DB}$ following their release into the soil and groundwater. There is also little known about the identity and impact of the products of biodegradation when yeast or fungi are used to degrade these compounds. Therefore, the main focus of this study is to continue the process of assessing the environmental impact of plasticizers using higher microorganisms. Specifically, the objectives of this work are to:

(1) investigate the ability of several yeast and fungi to degrade commonly used plasticizers as well as potential alternative plasticizers; 
(2) conduct detailed growth studies of the biodegradation of selected plasticizers in order to (i) monitor the growth of the microorganism in batch fermentations over time; (ii) evaluate the degree of biodegradation of the plasticizer; (iii) characterize the metabolites and determine their toxicity in the broth; and (iv) propose a mechanism of degradation of the plasticizer.

(3) assess whether the biodegradation of potential alternative plasticizers should be a major cause for concern. 


\section{MATERIALS AND METHODS}

\subsection{Microorganisms}

The four eukaryotes used in this research were obtained from the American Type Culture Collection (ATCC) and are listed in Table 2-1. These microorganisms were maintained in vials containing a sterilized solution of $20 \%$ glycerol (Sigma-Aldrich Co.) and Nutrient Broth mixture (8.0 $\mathrm{g} / \mathrm{L}$ Becton Dickinson Nutrient Broth in distilled water) at $-70^{\circ} \mathrm{C}$ in a freezer (REVCO, Model ULT1386) for a maximum period of one year. After this period new vials were prepared.

Table 2-1. Microorganisms used.

\begin{tabular}{|l|c|}
\hline \multicolumn{1}{|c|}{ Organism } & Optimal Growth $\left({ }^{\circ} \mathbf{C}\right)$ \\
\hline $\begin{array}{l}\text { Rhodotorula rubra J-96-1 } \\
\text { ATCC 9449 }\end{array}$ & 24 \\
\hline $\begin{array}{l}\text { Aspergillus niger } \\
\text { ATCC 9642-U }\end{array}$ & 24 \\
\hline $\begin{array}{l}\text { Aspergillus puniceus J-86-2 } \\
\text { ATCC 16800 }\end{array}$ & 24 \\
\hline $\begin{array}{l}\text { Candida bombicola } \\
\text { ATCC 22214 }\end{array}$ & 30 \\
\hline
\end{tabular}

Over short periods of time, the organisms were maintained on Nutrient Broth agar plates at $4{ }^{\circ} \mathrm{C}$ (Fisher brand ISOTEMP fridge). Periodically, new plates were made by streaking organisms that were growing in $100 \mathrm{~mL}$ of Nutrient Broth mixture $(8.0 \mathrm{~g} / \mathrm{L}$ in distilled water) in Erlenmeyer flasks $(500 \mathrm{~mL})$ as described below. The same preparation of the inoculums was used for the experiments for all four microorganisms. A sample 
vial containing the desired strain was taken from the $-70^{\circ} \mathrm{C}$ freezer and allowed to thaw for several hours. The thawed sample was then transferred to a sterile growth medium $(8.0 \mathrm{~g} / \mathrm{L}$ Nutrient Broth mixture in $100 \mathrm{~mL}$ of distilled water) after the vial had been vortexed (Fisher Vortex Genie 2) to resuspend the pellet. The organisms were incubated on a rotary incubator shaker (New Brunswick Scientific, Series 25) set at 250 RPM for a period of 2 days at the optimal temperatures specified in Table 2-1.

Inocula were prepared by adding $1.0 \mathrm{~mL}$ of this culture to a sterilized medium of modified mineral salt solution (MMSM), yeast extract ( $0.5 \mathrm{~g} / \mathrm{L}$ of Difco Yeast Extract), glucose $(2.5 \mathrm{~g} / \mathrm{L}$ or $1.65 \mathrm{~g} / \mathrm{L}$ of A\&C brand D-Glucose) or hexadecane $(2.5 \mathrm{~g} / \mathrm{L})$ and plasticizer ( $2.5 \mathrm{~g} / \mathrm{L})$ using sterile techniques in a laminar fume hood (The Baker Company, Model VBM600). The hexadecane and all of the plasticizers were obtained from Sigma-Aldrich Co. The composition of MMSM is shown in Table 2-2. The glucose was sterilized separately from the rest of the medium to prevent it from caramelizing.

Table 2-2. Composition of modified minimum mineral salt solution.

\begin{tabular}{|c|c|}
\hline Compound & Concentration (g/L) \\
\hline $\mathrm{NH}_{4} \mathrm{NO}_{3}$ & 4.0 \\
\hline $\mathrm{K}_{2} \mathrm{HPO}_{4}$ & 4.0 \\
\hline $\mathrm{Na}_{2} \mathrm{HPO}_{4}$ & 6.0 \\
\hline $\mathrm{MgSO}_{4} 7 \mathrm{H}_{2} \mathrm{O}$ & 0.2 \\
\hline $\mathrm{CaCl}_{2} 2 \mathrm{H}_{2} \mathrm{O}$ & 0.01 \\
\hline $\mathrm{FeSO}_{4} 7 \mathrm{H}_{2} \mathrm{O}$ & 0.01 \\
\hline $\mathrm{Na}_{2} \mathrm{EDTA}$ & 0.014 \\
\hline
\end{tabular}




\subsection{Shake Flask Experiments}

After 3 days of growth the inocula were ready for both the screening experiments and the growth study. Aliquots of the inocula were then transferred to the shake flasks using a 2-mL sterile pipette (Fisher Brand) and through the inoculation port for the reactor using a 5-mL plastic sterile syringe (Fisher Brand).

The shake flask screening experiments were all conducted using MMSM and 0.5 $\mathrm{g} / \mathrm{L}$ yeast extract with the addition of $2.5 \mathrm{~g} / \mathrm{L}$ plasticizer and $2.5 \mathrm{~g} / \mathrm{L}$ glucose or hexadecane as the co-substrates. The steam sterilization conditions for the Erlenmeyer flasks were $121^{\circ} \mathrm{C}$ and $20 \mathrm{psig}$ for approximately 30 minutes. The organisms grew in flasks on a rotary incubator shaker (New Brunswick Scientific, Series 25) set at 250 RPM. Two shakers were used, one set at $24{ }^{\circ} \mathrm{C}$ and the other at $30^{\circ} \mathrm{C}$ to accommodate the different optimal growing conditions of the four organisms.

After 8 days of growth, the contents of the flasks were sacrificed by extracting the complete broth $(100 \mathrm{~mL})$ with $50.0 \mathrm{~mL}$ of chloroform (A\&C brand) containing $0.01 \%$ (mass/volume ratio) of pentadecane (A\&C brand) as the internal standard. The extracts were then injected into the gas chromatograph (GC) for analysis (see section 2.5.1 for GC techniques). The area ratios of the plasticizer peaks to the internal standard peak were calculated and recorded. These final values were compared with results obtained from initial shake flask experiments in order to determine the degree of degradation of the plasticizers.

For the control experiments, the same growth media and sterilization conditions were used as in the experiments described earlier, but the flasks were not inoculated with 
the microorganisms. After they were prepared, the contents of the flasks were then extracted with chloroform and analyzed using the GC as described above.

\subsection{Batch Reactor Experiments}

The growth studies were carried out in a 2-L New Brunswick Scientific batch reactor (Figure 2-1). The reactor was maintained at constant temperature using a recirculating water bath (Haake, model FE2) and a stainless steel tube in a tube heat exchanger (New Brunswick Scientific). A condenser was used to prevent a loss of volume from the reactor due to evaporation and a dual-impeller agitator was used to provide mixing in the reactor. The agitator also had small holes drilled around the shaft at the height of the impellers to allow for aeration of the broth. The air inlet was fitted to an inline air filter (Millipore Millex-FG50, 0.2 $\mu \mathrm{L}$ ). A modified cover for the batch reactor sealed the reactor. This cover was machined from a 1-inch thick slab of Teflon with the ports drilled directly into it and was held in place by a 3-screw metal clamp. The Teflon cover was sealed to the reactor by a rubber gasket along the flat surface of the top of the reactor. A Teflon cover was necessary to ensure that no plasticizers would be available to leach into the reactor since plasticizers are not incorporated into this material.

To begin each growth study, $1.5 \mathrm{~L}$ of MMSM and $0.5 \mathrm{~g} / \mathrm{L}$ yeast extract were autoclaved in the batch reactor at $121^{\circ} \mathrm{C}$ and $20 \mathrm{psig}$ for a period of two hours. Once the reactor had cooled to $24{ }^{\circ} \mathrm{C}$ (the optimum growing conditions of $R$. rubra and A. niger), the presterilized glucose, the plasticizer and inoculum were injected into the batch reactor through a port using $5 \mathrm{~mL}$ sterile syringes (Fisher brand). Every growth study initially 
contained $2.5 \mathrm{~g} / \mathrm{L}$ of plasticizer and either $1.65 \mathrm{~g} / \mathrm{L}$ glucose with $R$. rubra or $2.5 \mathrm{~g} / \mathrm{L}$ glucose with A. niger.

Approximately every 8 hours, $15 \mathrm{~mL}$ samples were removed from the batch reactor through a sampling port while the reactor was kept at a constant temperature and mixing conditions. The samples were collected in a $30-\mathrm{mL}$ glass screw top vial with a Teflon seal (Fisher Brand) and stored at $4{ }^{\circ} \mathrm{C}$ until the completion of the growth study.

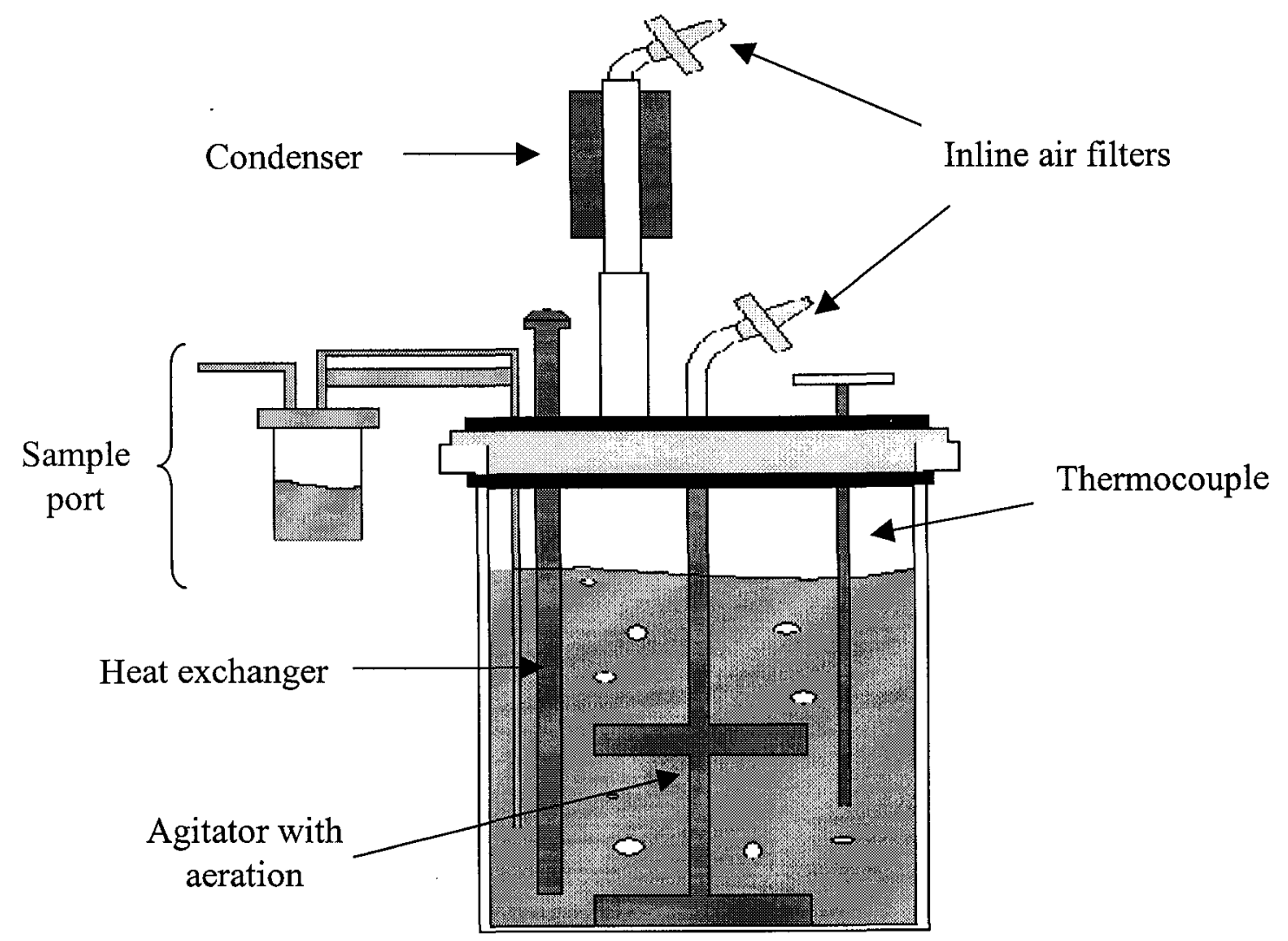

Figure 2-1. Schematic of the batch reactor. 


\subsection{Biomass Determination}

\subsubsection{Dry Weight Measurement}

A modified procedure of the standard dry weight analysis ${ }^{30}$ was used to initially measure the biomass. The samples in the $30-\mathrm{mL}$ vials were shaken and $10.0 \mathrm{~mL}$ were removed with a sterile pipette (Fisher brand) to make the measurements. The samples were placed in 30-mL Teflon centrifuge tubes (Fisher brand) and centrifuged (IEC, Model B-22M) for $10 \mathrm{~min}$ at 10,000 RPM at room temperature. The supernatant was then decanted and the pellet was washed with $10.0 \mathrm{~mL}$ of MMSM. After the two centrifugation and washing steps, the final pellet was resuspended in distilled water and placed in a preweighed aluminum dish. The dishes were then placed in an oven (Fisher Isotemp Oven 100 series, model $126 \mathrm{G}$ ) at $105{ }^{\circ} \mathrm{C}$ for a period of 48 hours. The dishes were cooled and the mass was obtained using an analytical balance (Mettler, model AE 160). The final measure was recorded as grams of dry biomass per liter of fermentation broth.

\subsubsection{Protein Concentration of Biomass}

The BIO-RAD DC Total Protein Assay (BIO-RAD Laboratories Inc.) was used to quantify the amount of biomass for all the growth studies. The samples in the $30-\mathrm{mL}$ vials were shaken and a volume of $10.0 \mathrm{~mL}$ was removed and placed in a $30 \mathrm{~mL}$ Teflon centrifuge tube (Fisher brand). The samples were centrifuged for 10 minutes at 10,000 RPM at room temperature. The supernatant was then decanted and the pellet was washed with $10.0 \mathrm{~mL}$ of MMSM. After the two centrifugation and washing steps, the final pellet was resuspended in $10.0 \mathrm{~mL}$ of MMSM. $0.1 \mathrm{~mL}$ of this sample was then mixed with 0.5 
$\mathrm{mL}$ of Reagent $\mathrm{A}$ (an alkaline copper tartrate solution) and vortexed for 5 seconds. Next, 4.0 mL of Reagent B (a dilute Folin Reagent) was added and the solution was vortexed for another 5 seconds. After waiting 20 minutes to allow the reaction to proceed, the absorbance of the solution was measured using a Ultra-Violet Spectrophotometer (Varian DMS 200) at a wavelength of $750 \mathrm{~nm}$.

Figure 2-2 shows the calibration curve used to convert absorbance to protein concentration using bovine albumin fraction V (USB Corporation). The procedure was followed as stated by BIO-RAD (BIO-RAD Laboratories Inc).

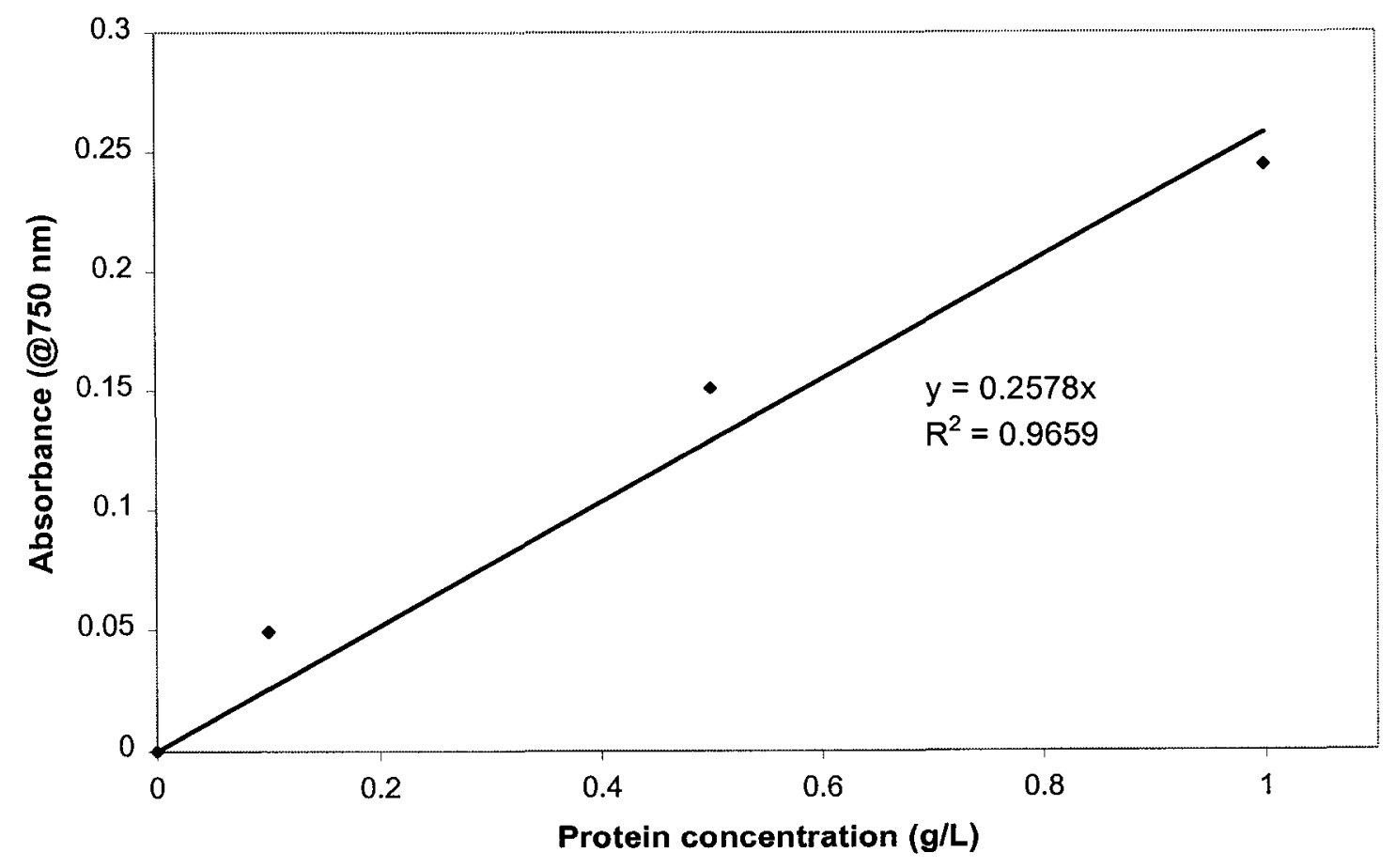

Figure 2-2. Calibration curve for protein concentration $(\bullet)$ 


\subsection{Analysis of Media Components}

\subsubsection{Plasticizers and Metabolites}

The following procedure was used to measure the concentration of plasticizers and metabolites in the various samples. The samples were well mixed and a 2-mL aliquot was removed with a $10-\mathrm{mL}$ sterile pipette (Fisher brand). This volume was then extracted with $3.0 \mathrm{~mL}$ of chloroform containing $0.01 \%$ (mass/volume ratio) pentadecane as an internal standard. The mixture was vortexed for one minute and the organic phase (extract) was removed by a Pasteur pipette into a 5-mL glass screw top vial with a Teflon seal (Fisher brand). The vials were stored at $-15^{\circ} \mathrm{C}$ until the samples were injected into the gas chromatograph (GC) or the gas chromatograph-mass spectrometry (GC-MS).

The gas chromatograph (GC) (HP5890 Series II) contained a SPB-5 column (Supelco) with a FID detector. The settings used for the GC are summarized in Table 23. Figures $2-3$ and $2-4$ are examples of calibration curves used to convert the $\mathrm{GC}$ area ratios of the plasticizer and metabolite peaks with respect to the internal standard peak area to concentration units.

The gas chromatograph-mass spectrometry (GC-MS) (Thermo Quest model TRACE GC 2000/ Finnigan POLARIS) contained a RTX-5 MS column (Restek) with an internal diameter of $0.25 \mathrm{~mm}$. The settings of the GC-MS are tabulated in Table 2-4. 
Table 2-3. Gas chromatography operation conditions

\begin{tabular}{|c|c|}
\hline Operation Conditions & Value \\
\hline Injection temperature & $220^{\circ} \mathrm{C}$ \\
\hline Initial column temperature & $65^{\circ} \mathrm{C}$ \\
\hline Temperature ramp rate & $10^{\circ} \mathrm{C} / \mathrm{min}$ \\
\hline Final column temperature & $320^{\circ} \mathrm{C}$ \\
\hline Detector temperature & $270^{\circ} \mathrm{C}$ \\
\hline Initial hold time & $0.1 \mathrm{~min}$ \\
\hline Final hold time & $5.0 \mathrm{~min}$ \\
\hline
\end{tabular}

Table 2-4. Gas chromatography-mass spectrometry operation conditions

\begin{tabular}{|c|c|}
\hline Operation Conditions & Value \\
\hline Injection temperature & $275^{\circ} \mathrm{C}$ \\
\hline Initial column temperature & $65^{\circ} \mathrm{C}$ \\
\hline Temperature ramp rate & $10^{\circ} \mathrm{C} / \mathrm{min}$ \\
\hline Final column temperature & $320^{\circ} \mathrm{C}$ \\
\hline Initial hold time & $0.1 \mathrm{~min}$ \\
\hline Final hold time & $5.0 \mathrm{~min}$ \\
\hline Start Mass Spec & $2.2 \mathrm{~min}$ \\
\hline Mass Spec Range & $10-350$ \\
\hline Transfer Line & $275^{\circ} \mathrm{C}$ \\
\hline Ion Source & $200^{\circ} \mathrm{C}$ \\
\hline
\end{tabular}




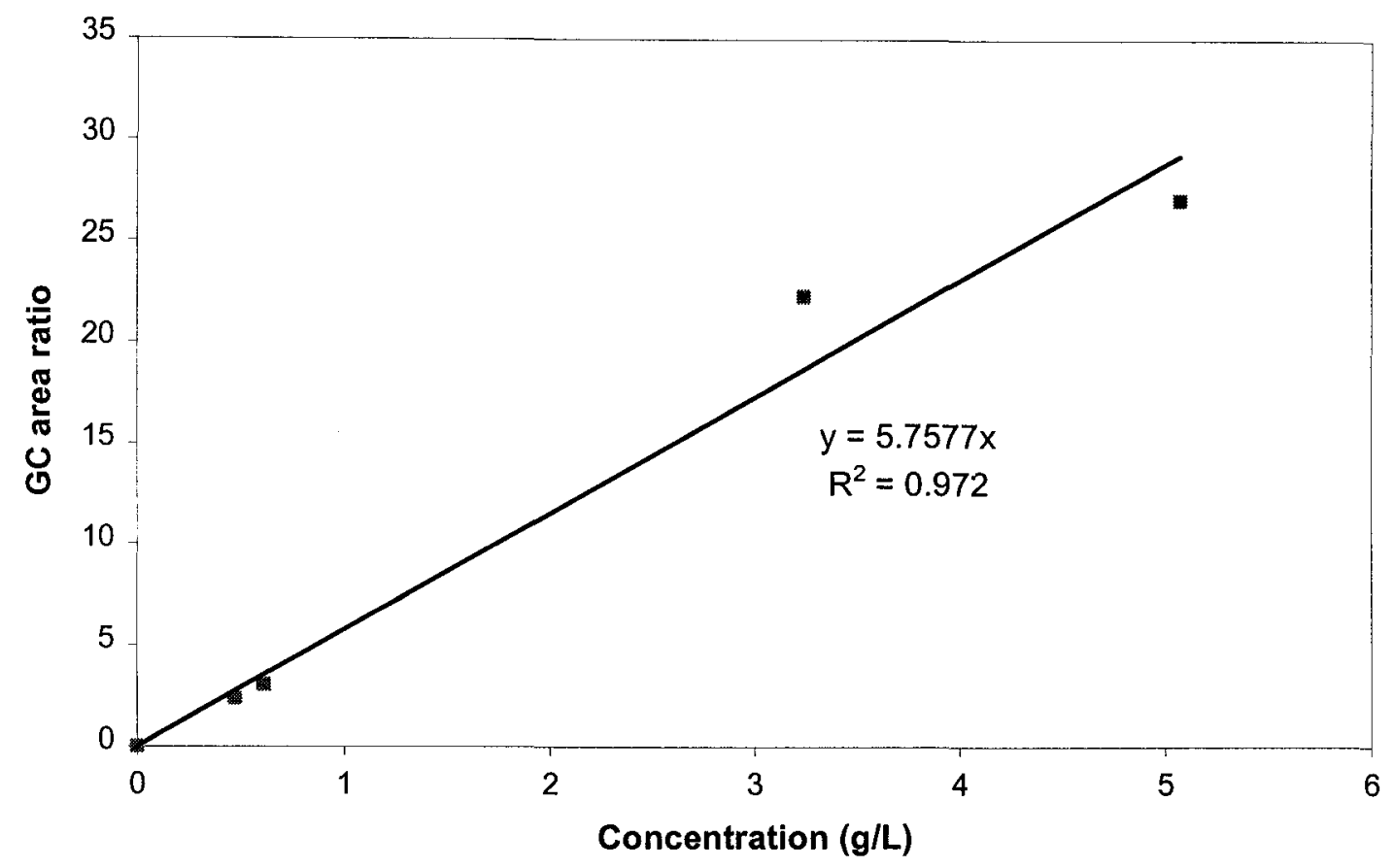

Figure 2-3. Calibration curve for di-ethylene glycol dibenzoate (ㅍ)

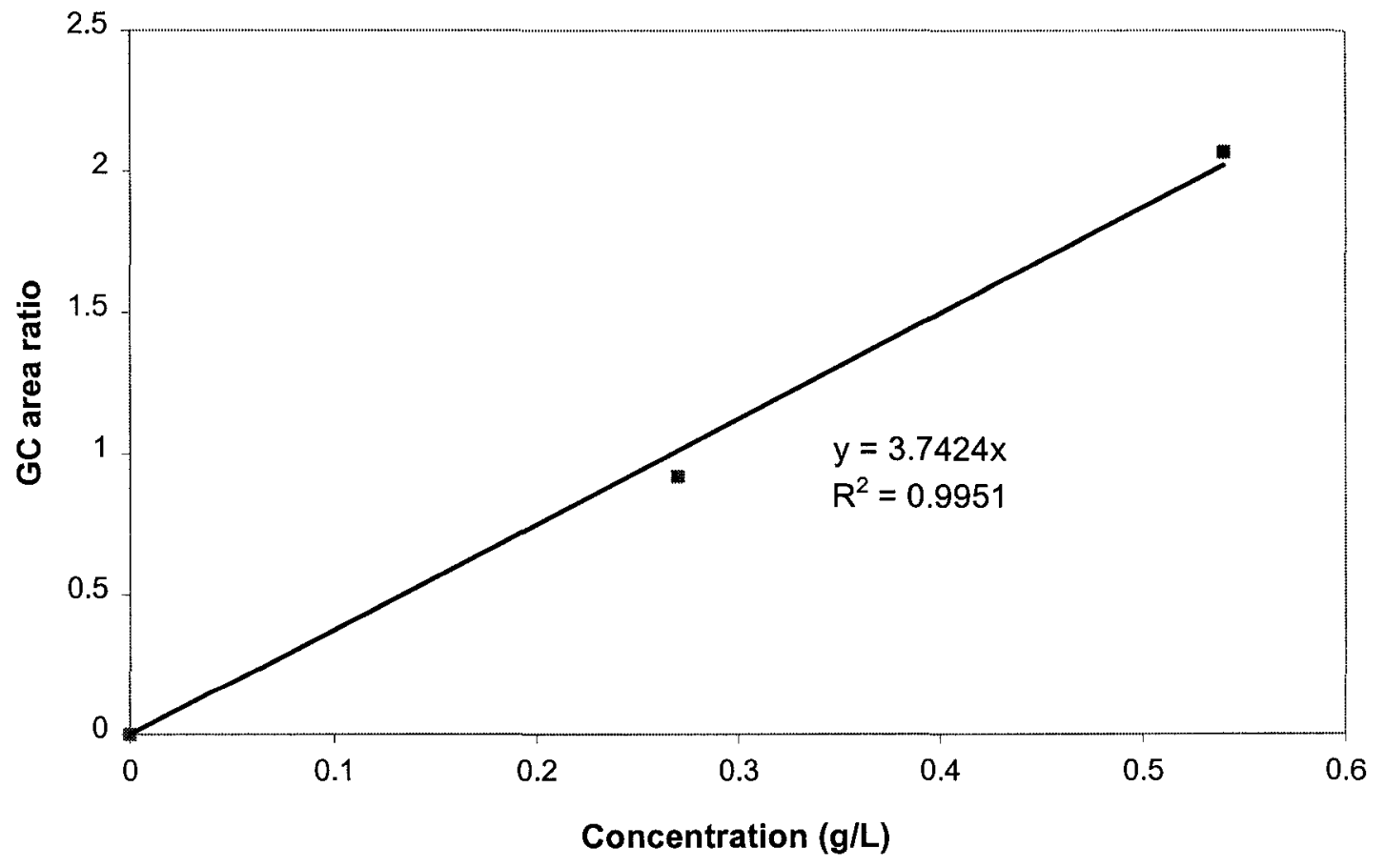

Figure 2-4. Calibration curve for the metabolite (1) from di-propylene glycol dibenzoate. 


\subsubsection{Glucose Concentration}

A modified procedure of the total carbohydrate assay (TCA $)^{30}$ was used to quantify the amount of glucose in every growth study. The samples in the $30-\mathrm{mL}$ vials were shaken and a volume of $10.0 \mathrm{~mL}$ was removed and placed in a Teflon centrifuge tube (Fisher Brand). The samples were centrifuged for 10 minutes at 10,000 RPM at room temperature. $1.0 \mathrm{~mL}$ of the supernatant was then diluted with $3.0 \mathrm{~mL}$ of distilled water in order to bring the absorbance of the assay mixture into the appropriate range (between $0.1-1.0$ ). A $0.1 \mathrm{~mL}$ sample of the diluted solution was further added to 0.9 $\mathrm{mL}$ of distilled water and then mixed with $1.0 \mathrm{ml}$ of a $5 \%$ phenol solution (Fisher brand). The mixture was vortexed for 10 seconds and then $5.0 \mathrm{~mL}$ of concentrated sulfuric acid (Fisher brand) was added. This final mixture was vortexed for 5 more seconds and then allowed to cool for 20 minutes before the absorbance was measured on a Ultra-Violet Spectrophotometer at a wavelength of $488 \mathrm{~nm}$.

Figure 2-5 shows the calibration curve used to convert absorbance to glucose concentration using prepared samples of known glucose concentrations. 


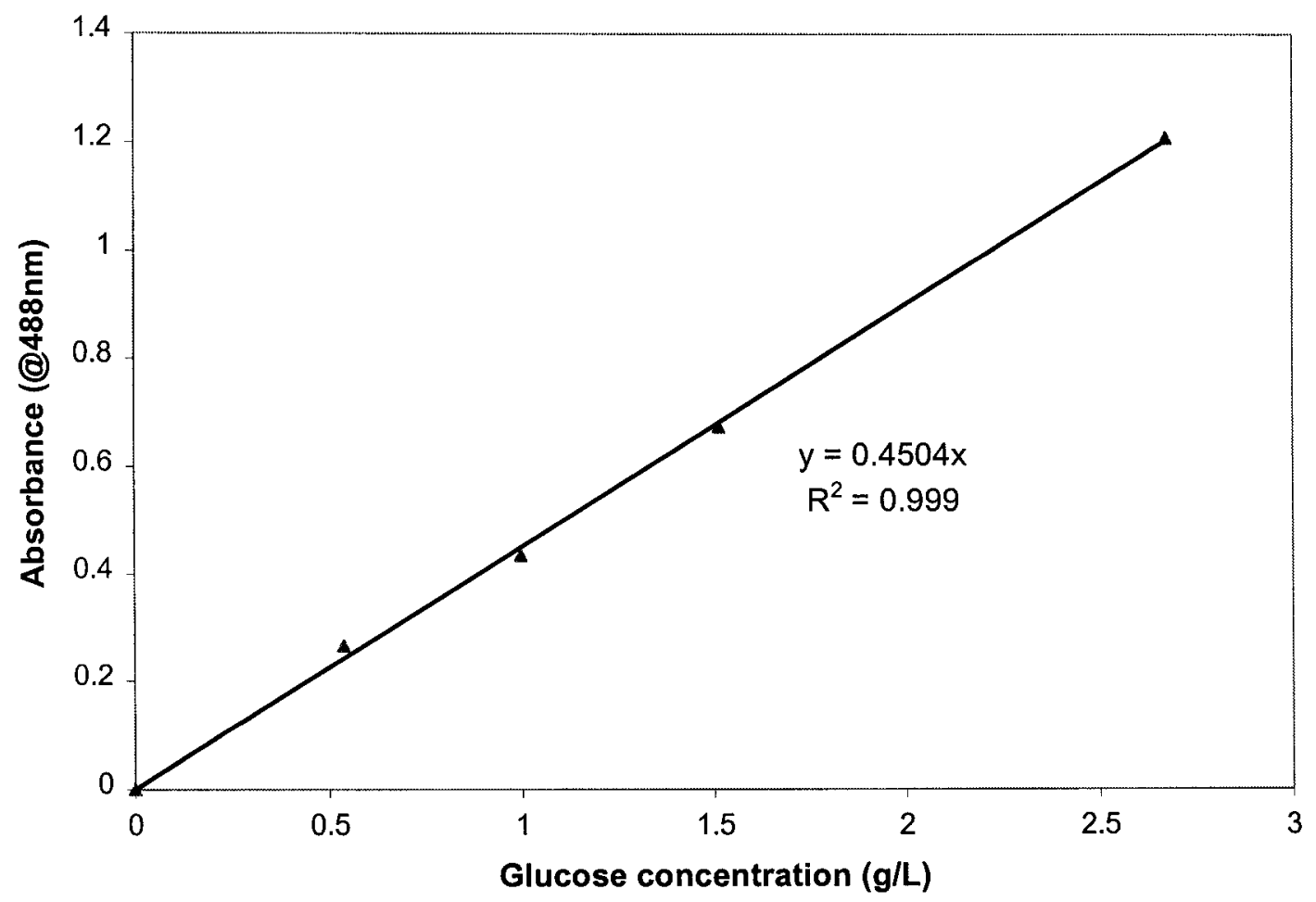

Figure 2-5. Calibration curve for glucose concentration ( $\mathbf{\Delta})$. 


\subsection{Toxicity Measurements}

\subsubsection{Microtox Assay}

The Microtox Assay was used to measure the toxicity of pure compounds and the relative change in toxicity of the broth from the growth studies. The samples in the 30 $\mathrm{mL}$ vials from the reactor were shaken and a volume of $10.0 \mathrm{ml}$ was removed and centrifuged at 10,000 RPM for 10 minutes at room temperature. The cell-free supernatants were decanted into $8 \mathrm{~mL}$ glass sample vials with Teflon seals (Fisher brand) and stored at $-15^{\circ} \mathrm{C}$ for eventual toxicity testing.

The Microtox Model 500 (Azur Environmental, formerly Microbics Corp.) relates toxicity to a decrease in luminescence by a marine organism, Vibrio fischeri NRRL B$11177^{11,22,40}$, which is proportional to the degree of inhibition caused by the compounds in the samples being tested. The theory on how luminescence relates to the biochemical pathways of higher organisms and the correlation between the Microtox and other commonly used toxicity tests is discussed in earlier work done by $\mathrm{Nalli}^{44}$.

One vial of the reconstituted organism can be used to test approximately 15 samples. This procedure requires only $2 \mathrm{~mL}$ of sample volume and allows 3 samples to be tested consecutively. The detailed procedure was followed as stated by the Microbics

Corporation $^{40}$. Basically, the assay measures the decrease of light output by the organism from a serial dilution (by a factor of 2) of the samples in question after the organism has been brought into contact with these potentially toxic samples. All the toxicity measures used the Basic Test setup in the Microtox software.

The measures of toxicity obtained from this test are $\mathrm{EC}_{50}$ (effective concentration causing a $50 \%$ decrease of light output, expressed in concentration units or percentage by 
volume) and $\mathrm{TU}_{50}$ values (toxicity units). The $\mathrm{EC}_{50}$ is obtained by plotting the concentration of the compounds in aqueous phase (from the serial dilutions of the samples) versus the output response on a logarithmic scale. The response is the relative change in light emitted by the organism after contacting the potentially toxic compounds in the samples. This parameter is called gamma, which is a ratio of light lost to the light remaining after the set time. When gamma is one, there is a $50 \%$ decrease in light emitted. This response corresponds to the effective concentration $\left(\mathrm{EC}_{50}\right)$. The lower the $\mathrm{EC}_{50}$ value, the higher the toxicity, thus, for a low $\mathrm{EC}_{50}$ value, a low concentration of the toxicant is required to elicit the $50 \%$ response from the test organism. The $\mathrm{TU}_{50}$ is directly proportional to the concentration of the toxicants and represents the number of dilutions that would have to be done to a sample to arrive at the $50 \%$ response level. Thus, a high $\mathrm{TU}_{50}$ corresponds to a high toxicity. It is calculated through $\mathrm{TU}_{50}=$ $100 \% / \mathrm{EC}_{50}$, where $\mathrm{EC}_{50}$ is expressed as a percentage.

The toxicities of samples analyzed in this study were expressed as $\mathrm{TU}_{50}$ values (in dilutions) due to the fact that the concentrations of the toxins in the mixtures were unknown. Thus, the measurement of $\mathrm{TU}_{50}$ can be used to measure the relative change in toxicity of the broth over time.

\subsubsection{Toxicity of Phases from Extraction Procedure}

The samples from the reactor were first prepared for toxicity testing using the procedure described in the previous subsection. However, each sample was divided in two. One sample was treated in the manner already described to obtain its toxicity. 2.0 $\mathrm{mL}$ of the second sample was diluted with $2.0 \mathrm{~mL}$ of MMSM and then extracted with 4.0 
$\mathrm{mL}$ of chloroform as described in the procedure for $\mathrm{GC}$ analysis. The aqueous raffinate $(2.0 \mathrm{~mL})$ was then collected, diluted with $2.0 \mathrm{~mL}$ of MMSM and prepared for analysis by the Microtox as described above.

The chemicals extracted into the chloroform were prepared for Microtox analyses in the following manner. $1.5 \mathrm{~mL}$ of the organic extracts were rotary evaporated to remove the chloroform and the left over residue was re-suspended in $3.0 \mathrm{~mL}$ of MMSM. 


\section{RESULTS}

\subsection{Preliminary Growth Studies}

Table 3-1 contains the results for the preliminary screening of four eukaryotic organisms degrading each of six different plasticizers in the presence of either glucose or hexadecane as a co-substrate. The microorganisms were selected based on their ability to degrade long-chained hydrocarbons.

The results demonstrate that all the plasticizers could be degraded to a certain extent by at least some of the organisms. Degradation of the plasticizers using hexadecane as a co-substrate was either complete or insignificant. Candida bombicola showed no ability to appreciably degrade any plasticizer, except di-propylene glycol dibenzoate (D(PG)DB), whereas Aspergillus puniceus was able to at least moderately degrade every plasticizer. When glucose was used as a co-substrate, all the organisms were able to degrade dioctyl phthalate (DOP), di-ethylene glycol dibenzoate (D(EG)DB) and $\mathrm{D}(\mathrm{PG}) \mathrm{DB}$ except for Aspergillus niger which showed no appreciable degradation with the latter. Bis (2-ethylhexyl) adipate (B(EH)A) and dioctyl terephthalate (DOTP) were degraded by all the organisms except for Rhodotorula rubra. However, R. rubra was the only organism that could significantly degrade both dibenzoate plasticizers using glucose as a co-substrate. 
Table 3-1. Degradation of plasticizers by eukaryotes in media containing plasticizer and a co-substrate ${ }^{\mathrm{a}}$.

\begin{tabular}{|c|c|c|c|c|c|c|}
\hline \multirow{2}{*}{ Plasticizers } & \multicolumn{2}{|c|}{$\begin{array}{c}\text { Candida } \\
\text { bombicola }\end{array}$} & \multicolumn{2}{c|}{$\begin{array}{c}\text { Aspergillus } \\
\text { puniceus }\end{array}$} & $\begin{array}{c}\text { Rhodotorula } \\
\text { rubra }\end{array}$ & $\begin{array}{c}\text { Aspergillus } \\
\text { niger }\end{array}$ \\
\cline { 2 - 7 } & Glucose & $\mathrm{C} 6$ & Glucose & $\mathrm{C} 6$ & Glucose & Glucose \\
\hline $\mathrm{B}(\mathrm{EH}) \mathrm{A}^{1}$ & + & - & ++ & + & - & ++ \\
\hline $\mathrm{DOTP}^{2}$ & ++ & - & + & + & - & + \\
\hline $\mathrm{DOP}^{3}$ & + & - & + & + & + & + \\
\hline $\mathrm{TCP}^{4}$ & + & - & - & + & - & - \\
\hline $\mathrm{D}(\mathrm{EG}) \mathrm{DB}^{5}$ & + & - & ++ & ++ & ++ & ++ \\
\hline $\mathrm{D}(\mathrm{PG}) \mathrm{DB}^{6}$ & + & + & + & ++ & ++ & - \\
\hline
\end{tabular}

a. Significant degradation (75\%-100\% disappeared) $(++)$; moderate degradation $(+)$; no appreciable degradation (-)

1. $\mathrm{B}(\mathrm{EH}) \mathrm{A}=\mathrm{Bis}$ (2-ethylhexyl) adipate

2. DOTP $=$ Dioctyl terephthalate

3. $\mathrm{DOP}=$ Dioctyl phthalate

4. $\mathrm{TCP}=$ Tri-cresyl phosphate

5. $\mathrm{D}(\mathrm{EG}) \mathrm{DB}=$ Di-ethylene glycol dibenzoate

6. $\mathrm{D}(\mathrm{PG}) \mathrm{DB}=\mathrm{Di}$-propylene glycol dibenzoate 


\subsection{Batch Fermentations}

\subsubsection{Plasticizer Sampling Error}

There were difficulties in obtaining a representative, homogeneous sample of the reactor broth in all of the batch fermentations. Due to the very low solubility of plasticizer in the aqueous media, there were two liquid phases in the reactor. Figure 3-1 shows the growth curve and concentration of plasticizer for a typical batch fermentation system. Initially there was $2.5 \mathrm{~g} / \mathrm{L}$ of plasticizer in the reactor (i.e., the oil phase) but early in the experiment, due to inadequate mixing, the samples taken often contained unrealistically low amounts of this compound. This problem, which was observed in all the growth studies carried out, was overcome as soon as there was an appreciable amount of biomass in the reactor. Gibbons and Alexander have reported that the biomass of microorganisms that can grow on sparingly substrates organic chemicals often acts as an

emulsifier $^{20}$. This would disperse the oil phase in the aqueous phase, resulting in a more homogenous mixture. Therefore, in all of the growth studies presented in the results, the GC data for the plasticizers were only reported for samples taken after forty hours of growth. The initial amount of plasticizer added to the reactor $(2.5 \mathrm{~g} / \mathrm{L})$ was assumed to be the actual concentration at the beginning of the fermentation. 


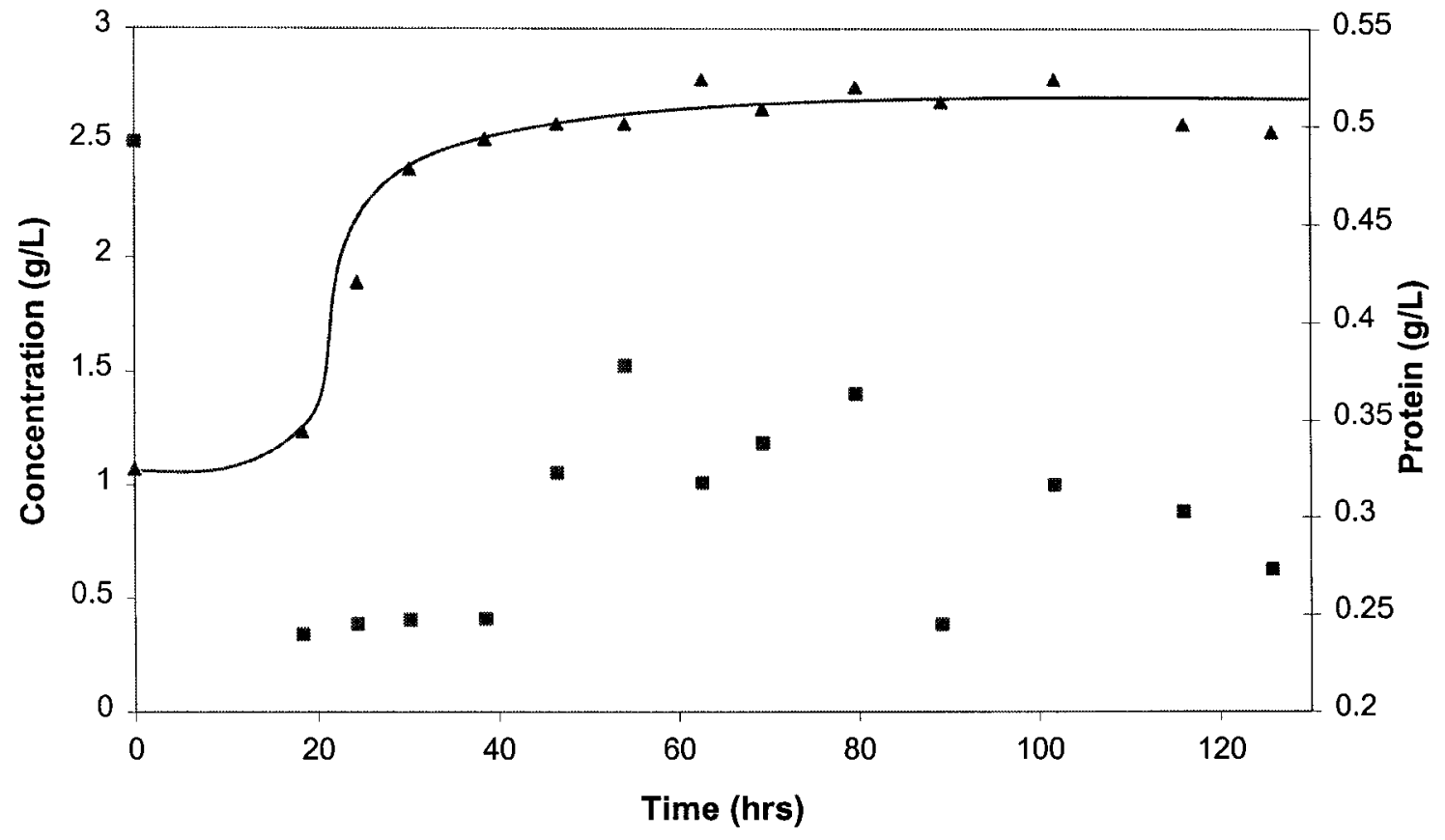

Figure 3-1. Growth of $R$. rubra expressed as protein $(\boldsymbol{\Delta})$, on a medium containing di-propylene glycol dibenzoate (E) and glucose. 


\subsubsection{Use of Measurement of Total Protein for Biomass}

Figure 3-2 shows the growth of $R$. rubra on glucose in the presence of dipropylene glycol dibenzoate. It demonstrates that the measurement of the total protein in the broth is at least as good an indication of growth as dry weight measurements. Dry weight measurements are particularly difficult and inaccurate for systems containing an

insoluble substrate ${ }^{53}$ due to the entrainment of oil in the biomass pellet. Protein measurements are relatively quick and simple to perform and eliminate the problems encountered with the dry weight test due to the interference of the viscous, insoluble plasticizer. In all subsequent graphs, the amount of protein in the broth was used as an indication of growth.

Exponential growth was observed for the microorganisms used in all the growth studies. Typically, the stationary phase was reached in approximately forty hours and continued for the duration of these fermentations (120 to140 hours). Growth coincided well with the decrease in glucose of the systems but a considerable amount of glucose was still present at the end of all the experiments. 


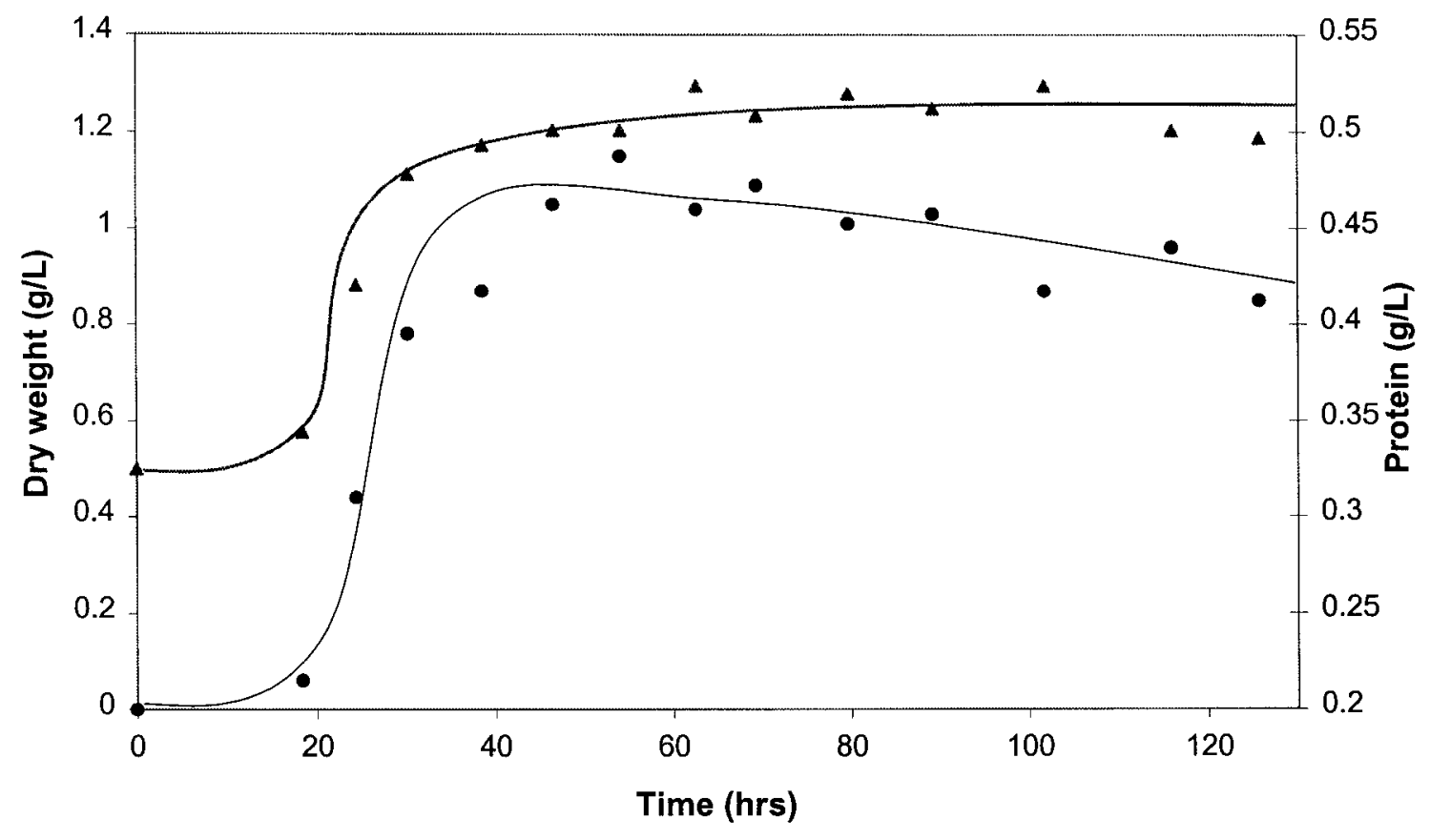

Figure 3-2. Dry weight (O) and protein (A) for the growth of $R$. rubra in a medium containing di-propylene glycol dibenzoate and glucose. 


\subsubsection{Fermentations}

A typical batch fermentation media contained $0.25 \%$ of one plasticizer and either $0.165 \%$ or $0.25 \%$ glucose, expressed in volumetric ratios. The degradation and toxicity studies were all found to be reproducible but only some duplicate examples are shown here.

The concentrations of the plasticizers were all determined by gas chromatography (GC). As the plasticizers were degraded, new peaks appeared in some of these chromatographs.

Figure 3-3 shows the growth of $R$. rubra on a medium containing solely glucose. This experiment was used as the control fermentation to compare with all the other growth studies with this microorganism. Relative to other observable changes, the toxicity of the cell-free broth was considered to be negligible throughout the fermentation

The results shown in Figures 3-4 (a) and 3-4 (b) are for the growth study in the presence of $\mathrm{D}(\mathrm{PG}) \mathrm{DB}$. Approximately $80 \%$ of the plasticizer was degraded throughout the course of the fermentation and as the concentration of the plasticizer decreased, a cluster of new peaks appeared (Figure 3-15) in the GC of the extracts of the broth. This cluster of peaks will be shown to be due to the formation of metabolites from the incomplete degradation of the plasticizer. The metabolites appeared in the samples after the concentration of $\mathrm{D}(\mathrm{PG}) \mathrm{DB}$ had started to decrease and then seemed to level off at about the same time that the degradation of the plasticizer seemed to slow. 


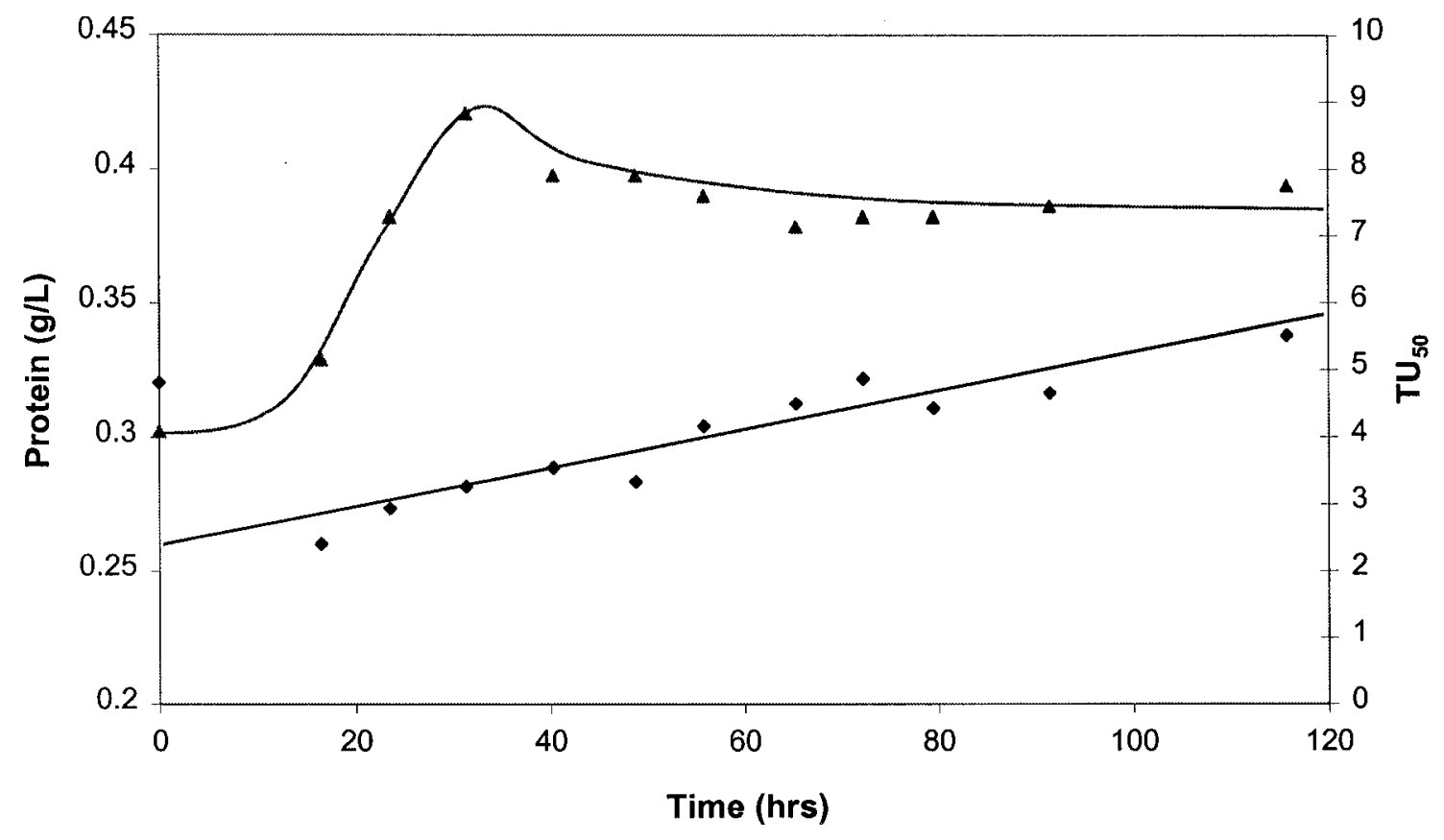

Figure 3-3. Growth of $R$. rubra expressed as protein (A) and Toxicity ( $\bullet$ ), on a medium containing solely glucose. 

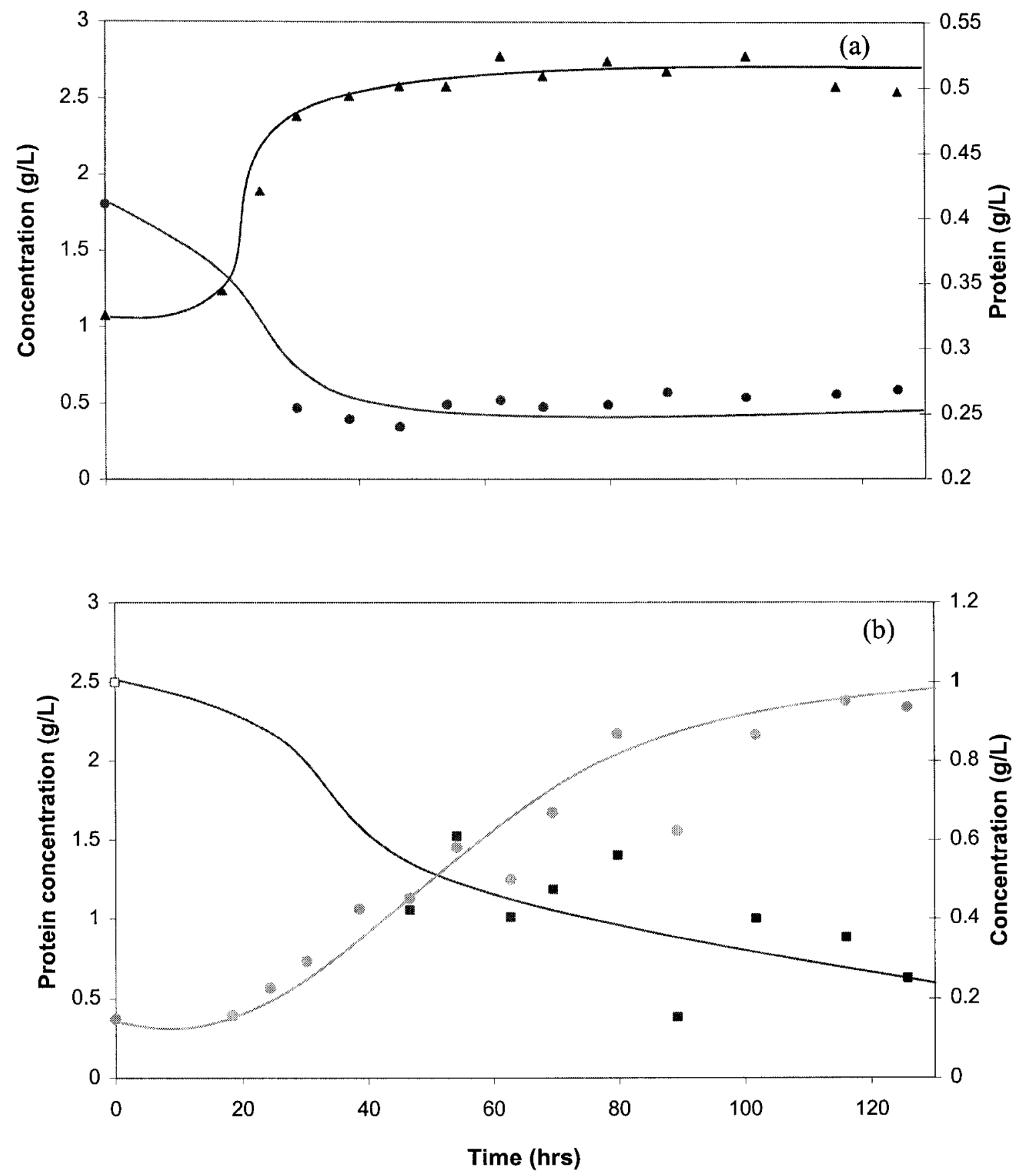

Figure 3-4. For a system using $R$. rubra: (a) protein (A) and glucose concentration ( ) (b) concentration of di-propylene glycol dibenzoate ( $\mathbf{G}$ ) and a pattern of metabolites (w). The initial amount of plasticizer added to the reactor is also shown ( $\square$ ). 
Figure 3-5 contains the toxicity data for samples of the cell-free broth and the concentration of the metabolites. The initial toxicity of the plasticizer had a $\mathrm{TU}_{50}$ of 20 . The toxicity remained at this value for a short time at the beginning of the experiment and then increased dramatically once the metabolites began to accumulate in the reactor medium. The $\mathrm{TU}_{50}$ reached a maximum of approximately 140 and then decreased as the concentration of the metabolites began to decrease slightly from a maximum value of 1.0 g/L. Figure 3-6 contains data from a second study with $D(P G) D B$ using identical conditions. The overall trends are very similar but there are some variations. The correspondence between the change in toxicity of the broth and the concentration of the metabolites is more obvious in this graph. In this example, after the maximum, the simultaneous decrease in toxicity and metabolite concentration was more pronounced. 


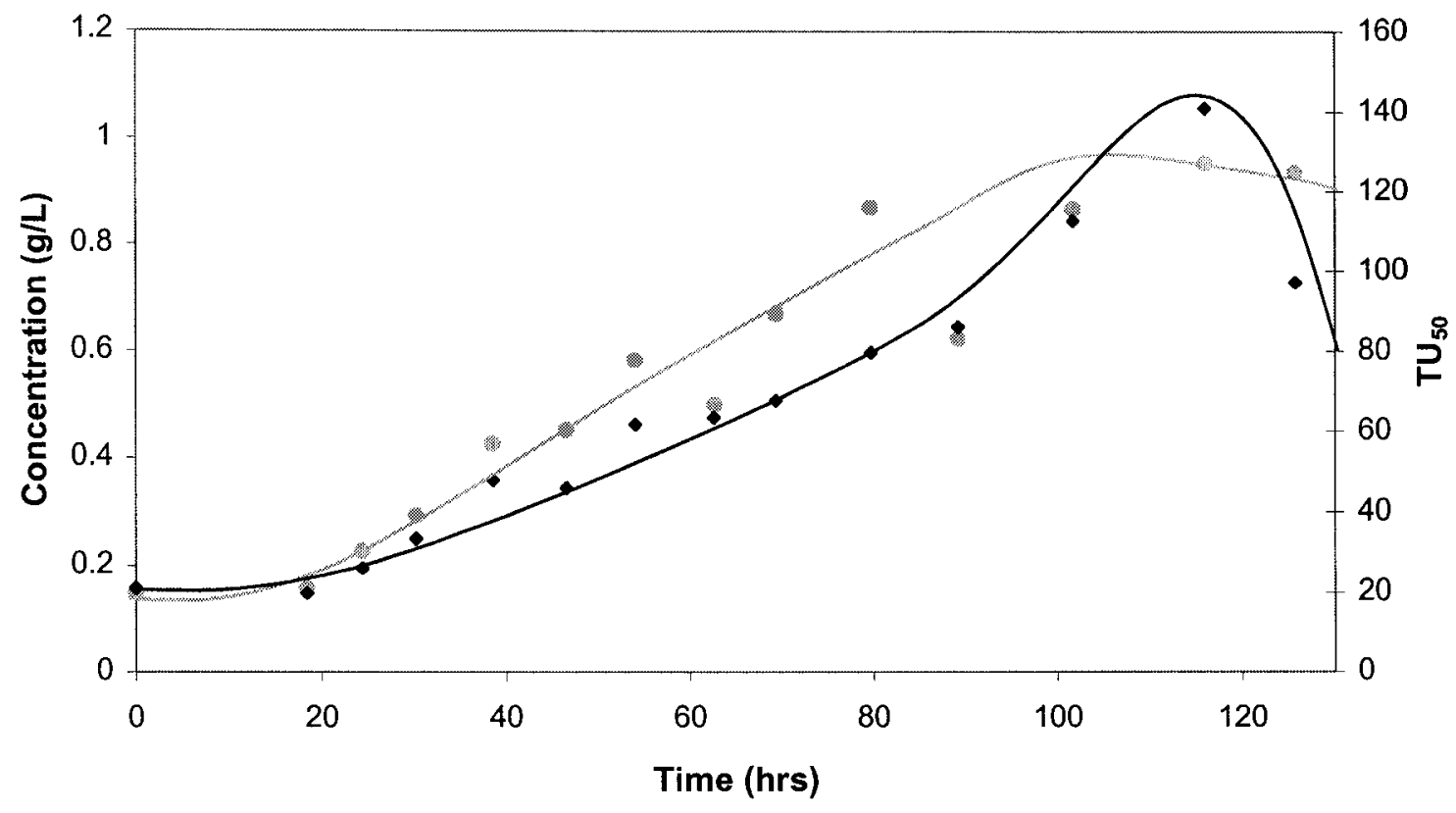

Figure 3-5. The concentration of a pattern of metabolites (b) and toxicity $(\checkmark)$ for the system represented in Figure 3-4 (a), (b).

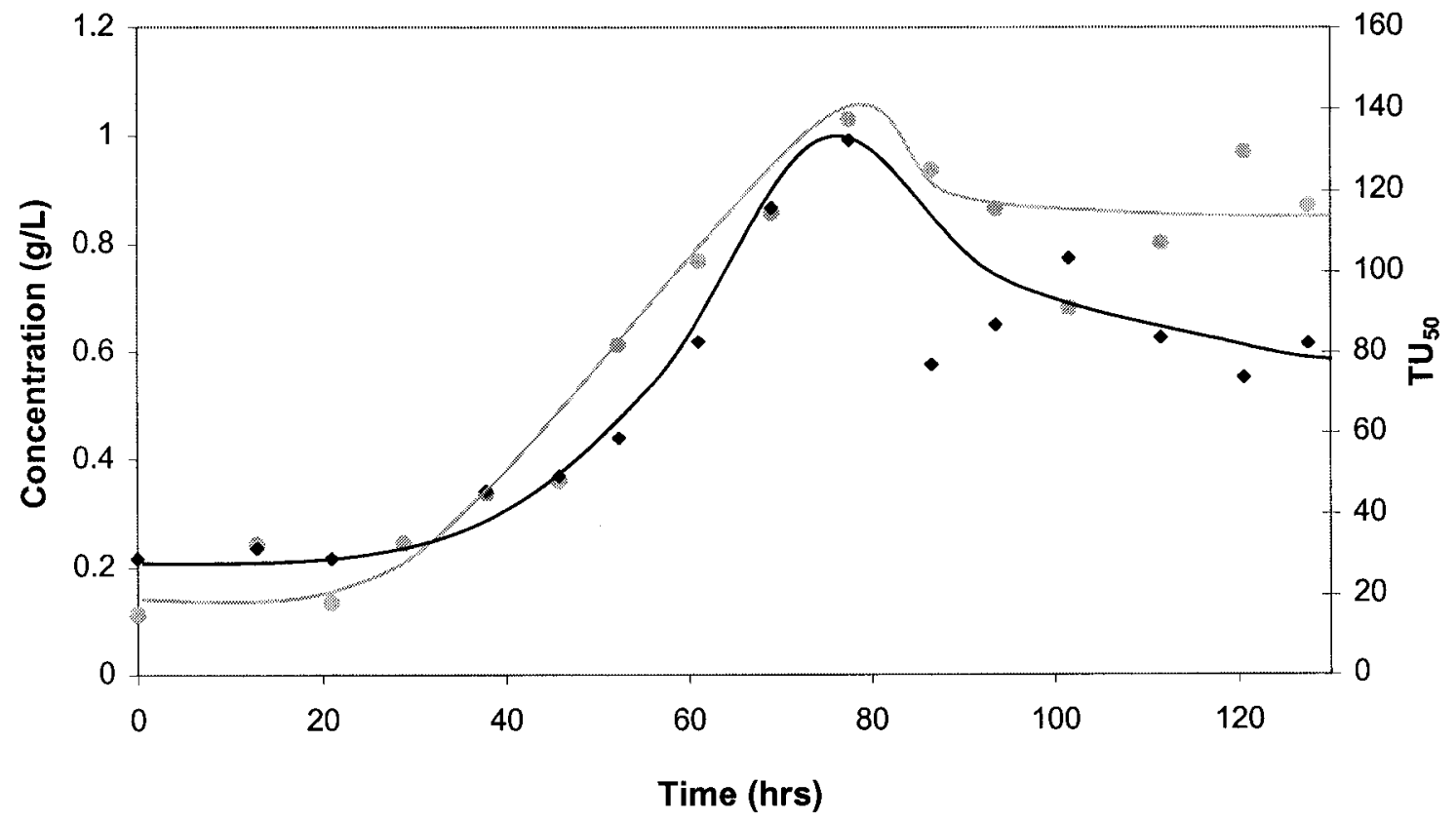

Figure 3-6. The concentration of a pattern of metabolites ( ) and toxicity ( ) from the repeat of the experiment represented in Figure 3-4 (a), (b). 
Figures 3-7 (a) and 3-7 (b) show that the degradation of D(EG)DB with R. rubra was more extensive than observed for the di-propylene glycol analogue. As D(EG)DB was degraded there was a new, single peak in the GC spectra of samples taken from the broth. This metabolite accumulated in the system until it reached a maximum GC area ratio of five. Figure 3-8 shows that the toxicity peaked at a $\mathrm{TU}_{50}$ above 70 and then decreased as the metabolite seemed to plateau.

Similar trends were observed when A. niger was used to degrade $\mathrm{D}(\mathrm{EG}) \mathrm{DB}$ (Figures 3-9 (a) and 3-9 (b)). Once again, as the plasticizer was degraded, evidence of a metabolite began to appear in the GC traces and its retention time was identical to the one observed with $R$. rubra. The metabolite appeared in the middle of the fermentation and, over a forty-hour period, increased to a $\mathrm{GC}$ area ratio of 0.5 and then decreased. The toxicity, shown in Figure 3-10, followed very closely with the metabolite and peaked at a $\mathrm{TU}_{50}$ of 25 . 

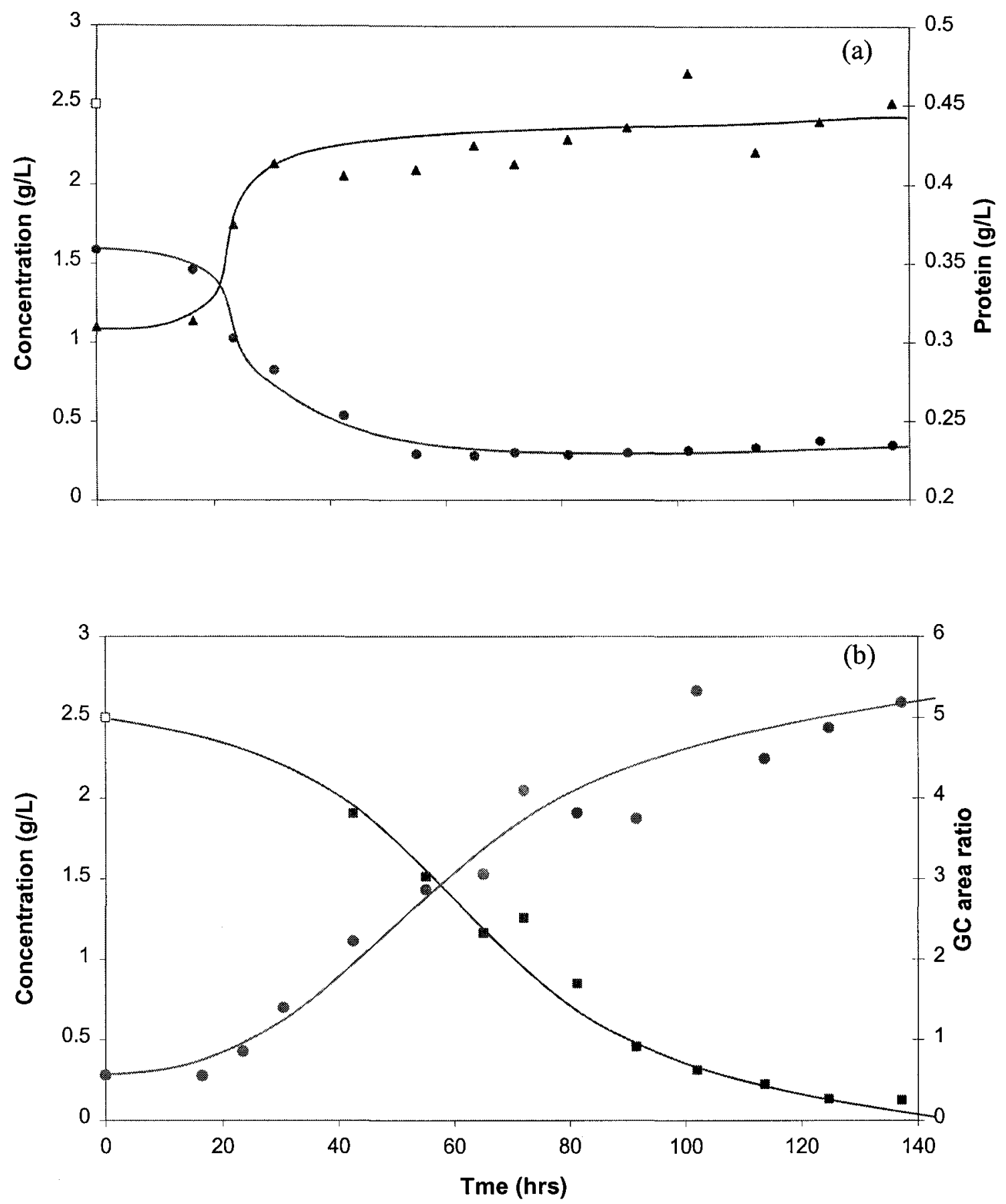

Figure 3-7. For a system using $R$. rubra: (a) protein ( $\mathbf{\Delta}$ ) and glucose concentration (-) (b) concentration of di-ethylene glycol dibenzoate ( $\mathbf{(})$ and a metabolite (-). The initial amount of plasticizer added to the reactor is also shown ( $\square$ ). 


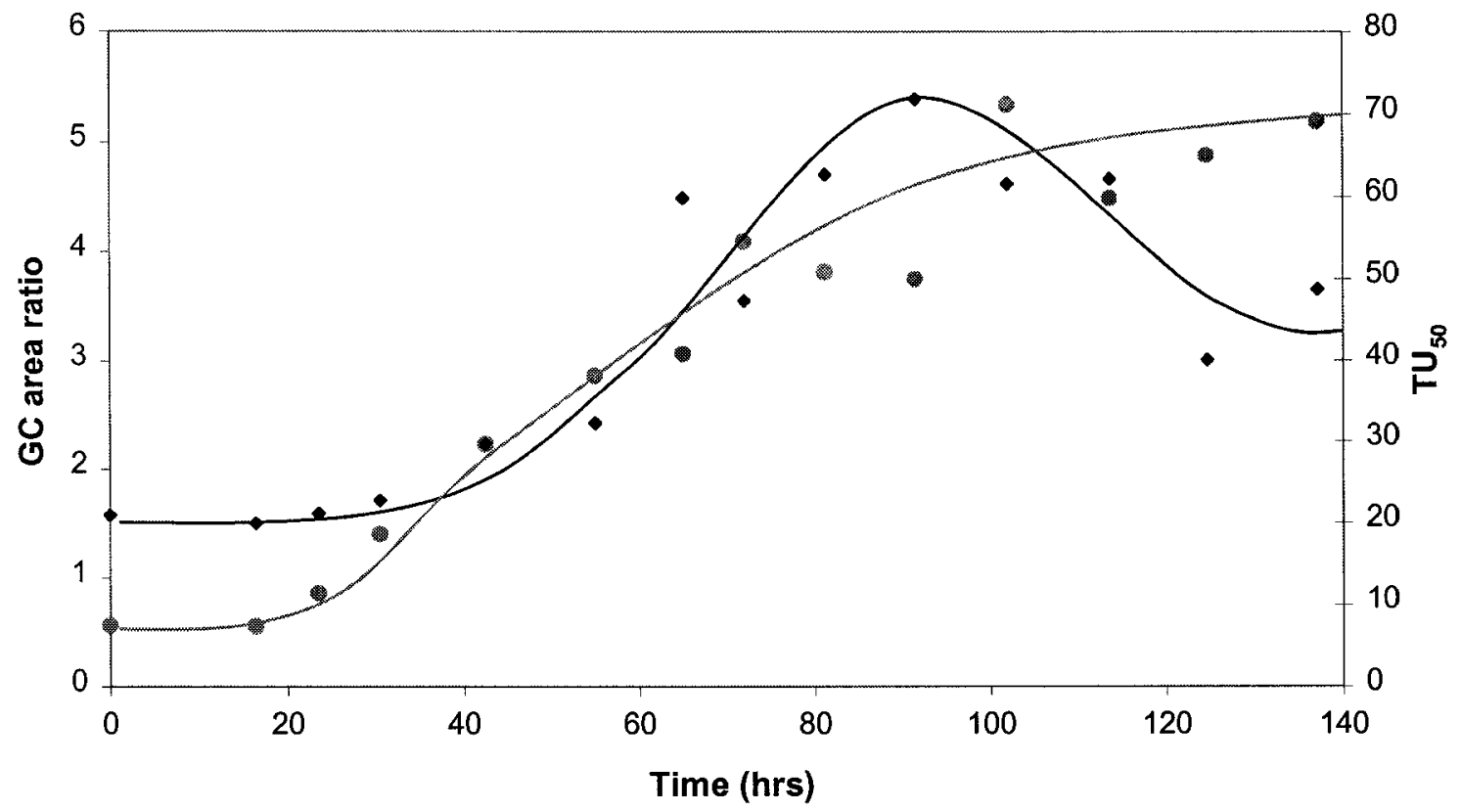

Figure 3-8. GC area ratio of a metabolite ( ) and toxicity $(\diamond)$ for the system represented in Figure 3-7 (a), (b). 

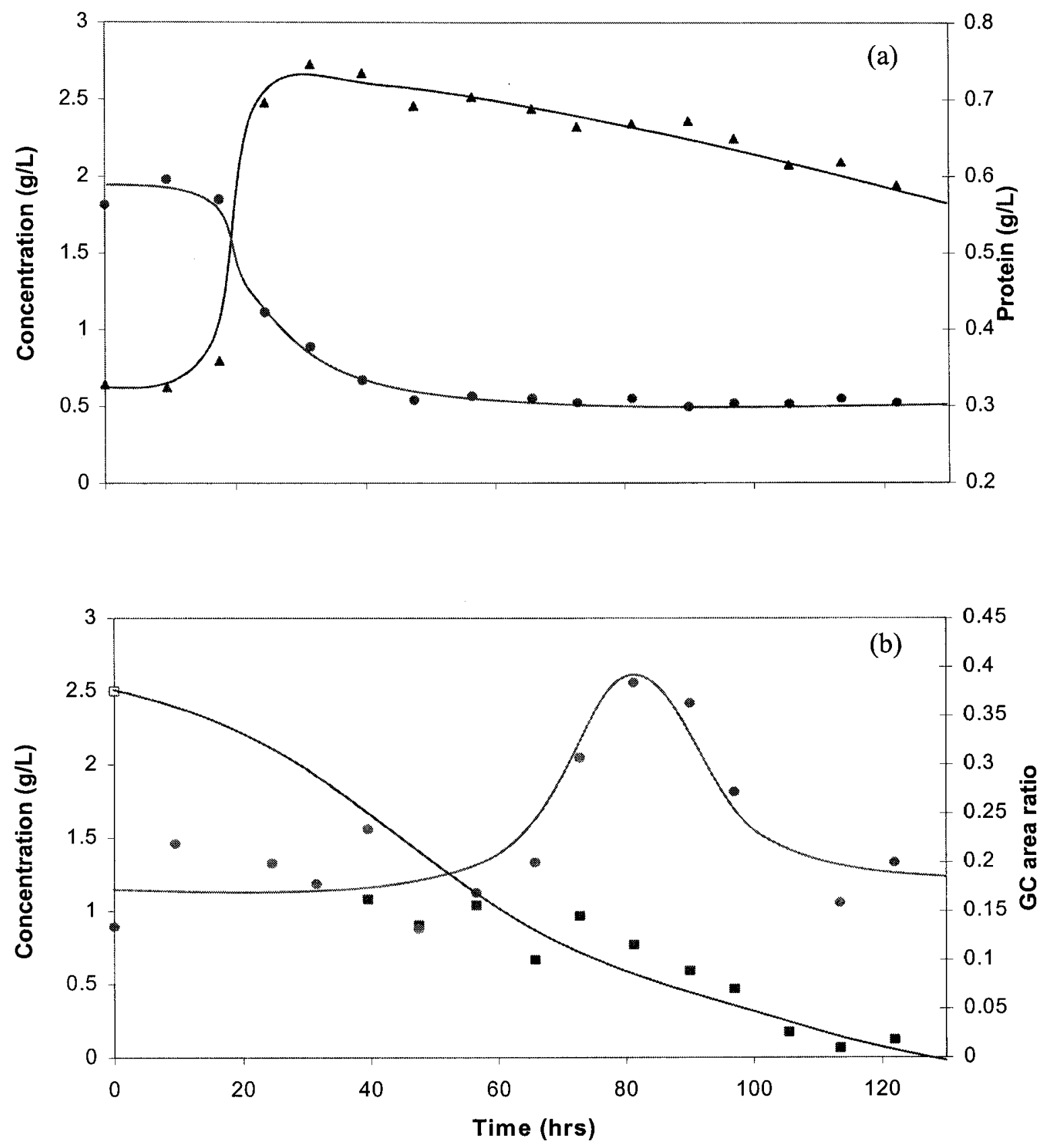

Figure 3-9. For a system using $A$. niger: (a) protein $(\boldsymbol{\Delta})$ and glucose concentration ( $)$ (b) concentration of di-ethylene glycol dibenzoate $(\mathbf{a})$ and a metabolite ( ). The initial amount of plasticizer added to the reactor is also shown (口). 


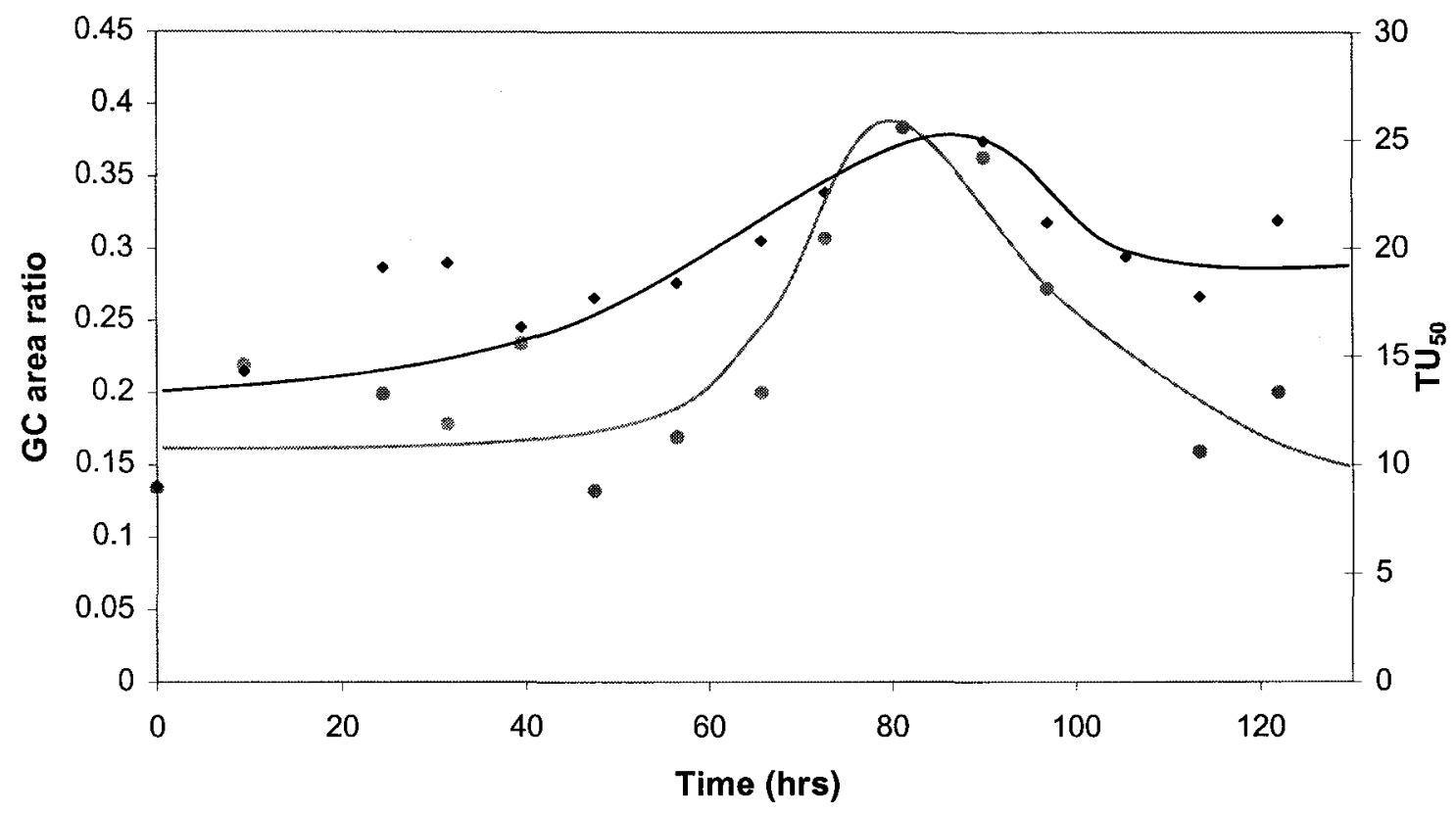

Figure 3-10. GC area ratio of a metabolite ( $)$ and the toxicity $(\diamond)$ for the system represented in Figure 3-9 (a), (b). 
The results for the degradation of the three plasticizers $(\mathrm{B}(\mathrm{EH}) \mathrm{A}, \mathrm{DOP}$, and DOTP) with $R$. rubra are shown in Figures 3-11 (a), 3-12 (a) and 3-13 (a). There was substantial degradation of all three plasticizers throughout the fermentation; however, no metabolites were observed in the GC spectra of the samples of the broth. The stationary phase was reached in thirty hours when the organism was grown on glucose and each of B(EH)A and DOP. Figures 3-11 (b), 3-12 (b), and 3-13 (b) show the toxicity data as well as the decrease in the amount of plasticizer for all three experiments. The initial toxicities of the plasticizers were found to be below a $\mathrm{TU}_{50}$ of 2.5 , which is considered to be almost negligible. In fact, the toxicity observed with DOTP as a substrate stayed below 2.5 throughout the entire fermentation. A small increase, followed by a decrease in toxicity, was observed at the beginning of the fermentations of both $\mathrm{B}(\mathrm{EH}) \mathrm{A}$ and $\mathrm{DOP}$. This trend was more evident for the DOP system, but in both cases the toxicity stayed between a $\mathrm{TU}_{50}$ of 2 and 7. 

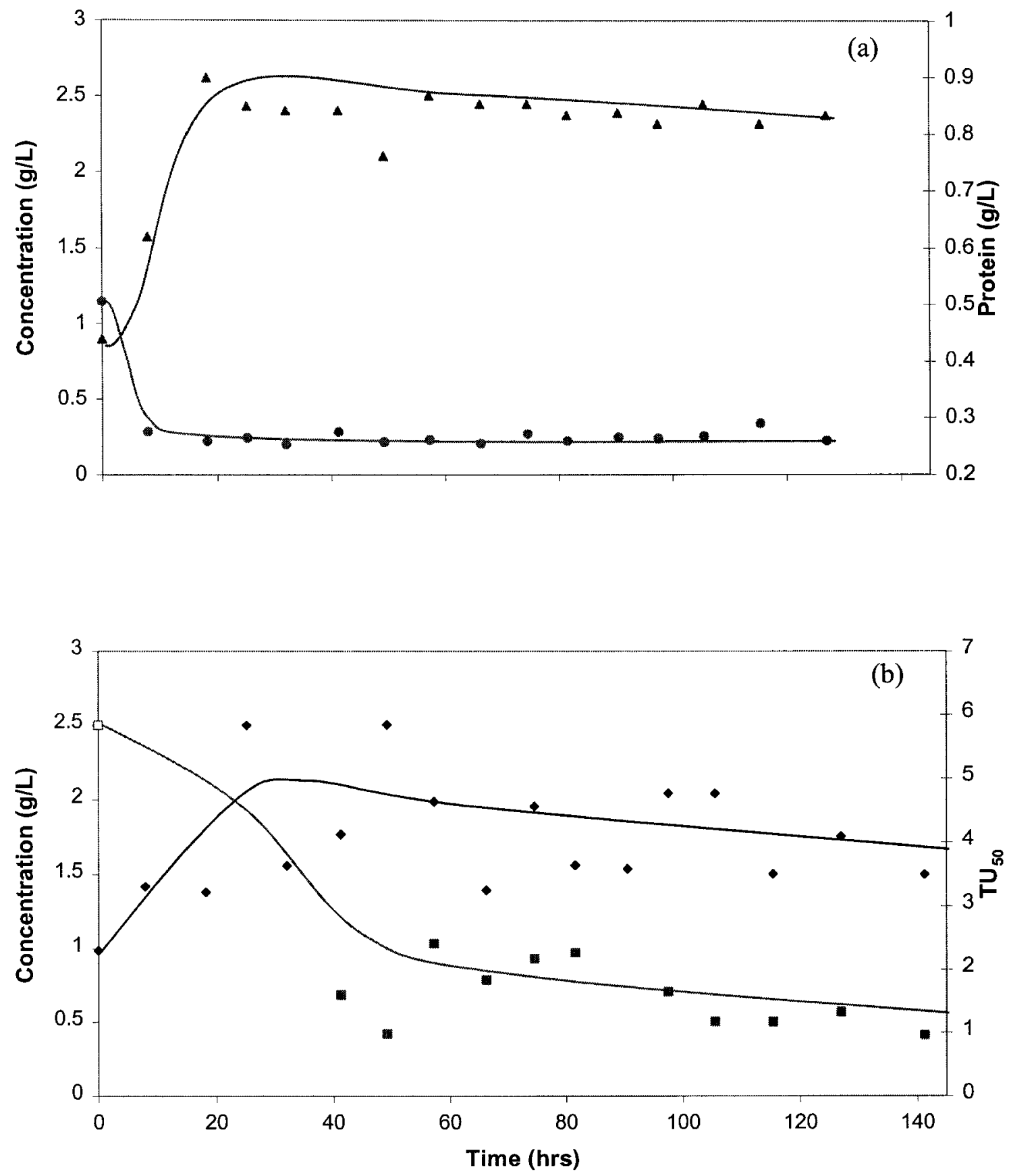

Figure 3-11. For a system using R. rubra: (a) protein ( $\mathbf{A})$ and glucose concentration ( ) (b) concentration of bis (2-ethylhexyl) adipate ( $\mathbf{\square})$ and toxicity $(\diamond)$. The initial amount of plasticizer added to the reactor is also shown ( $\square$ ). 

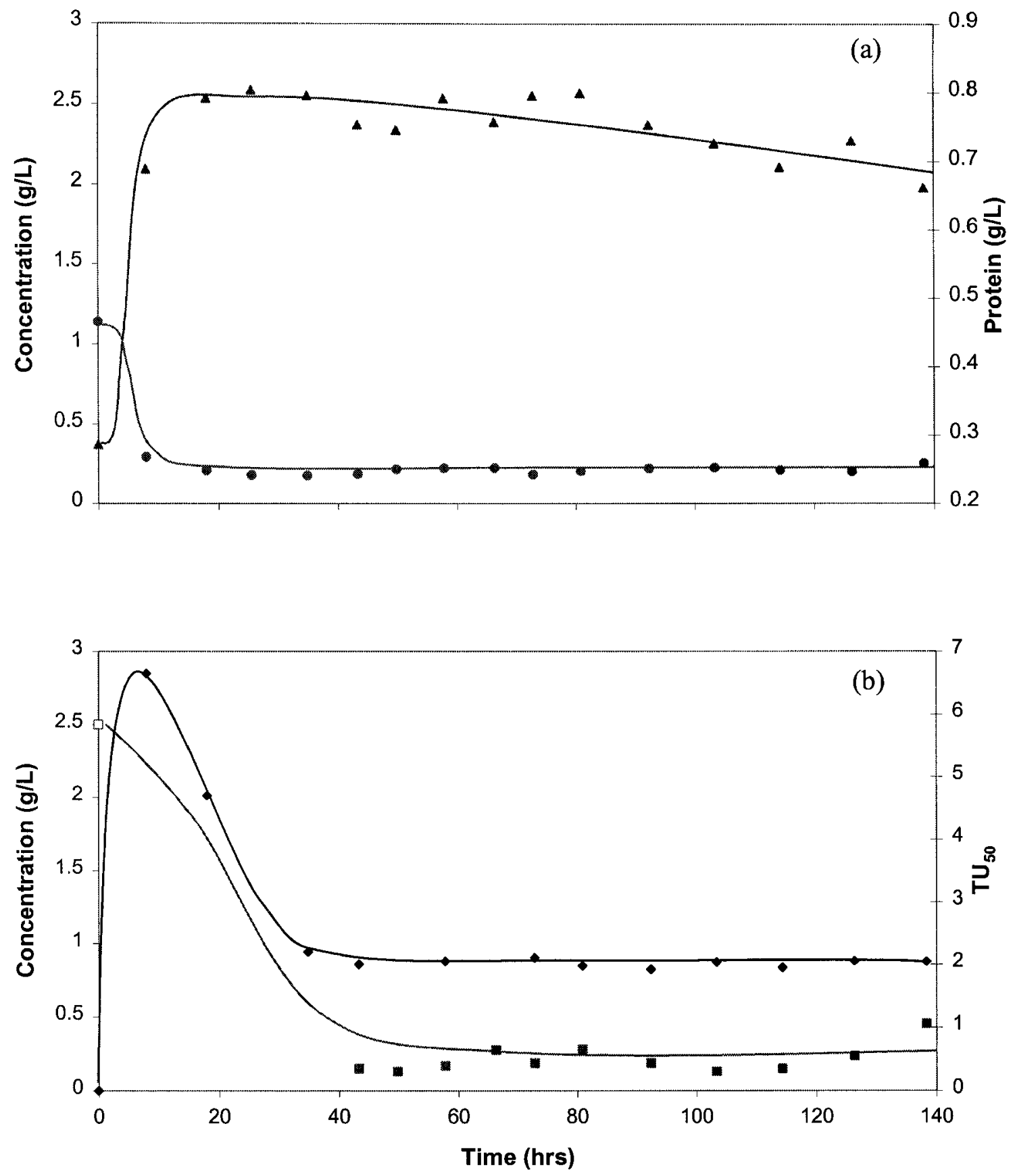

Figure 3-12. For a system using $R$. rubra: (a) protein (A) and glucose concentration ( $)$ (b) concentration of dioctyl phthalate $(\boldsymbol{D})$ and toxicity $(\bullet)$. The initial amount of plasticizer added to the reactor is also shown ( $\square$ ). 

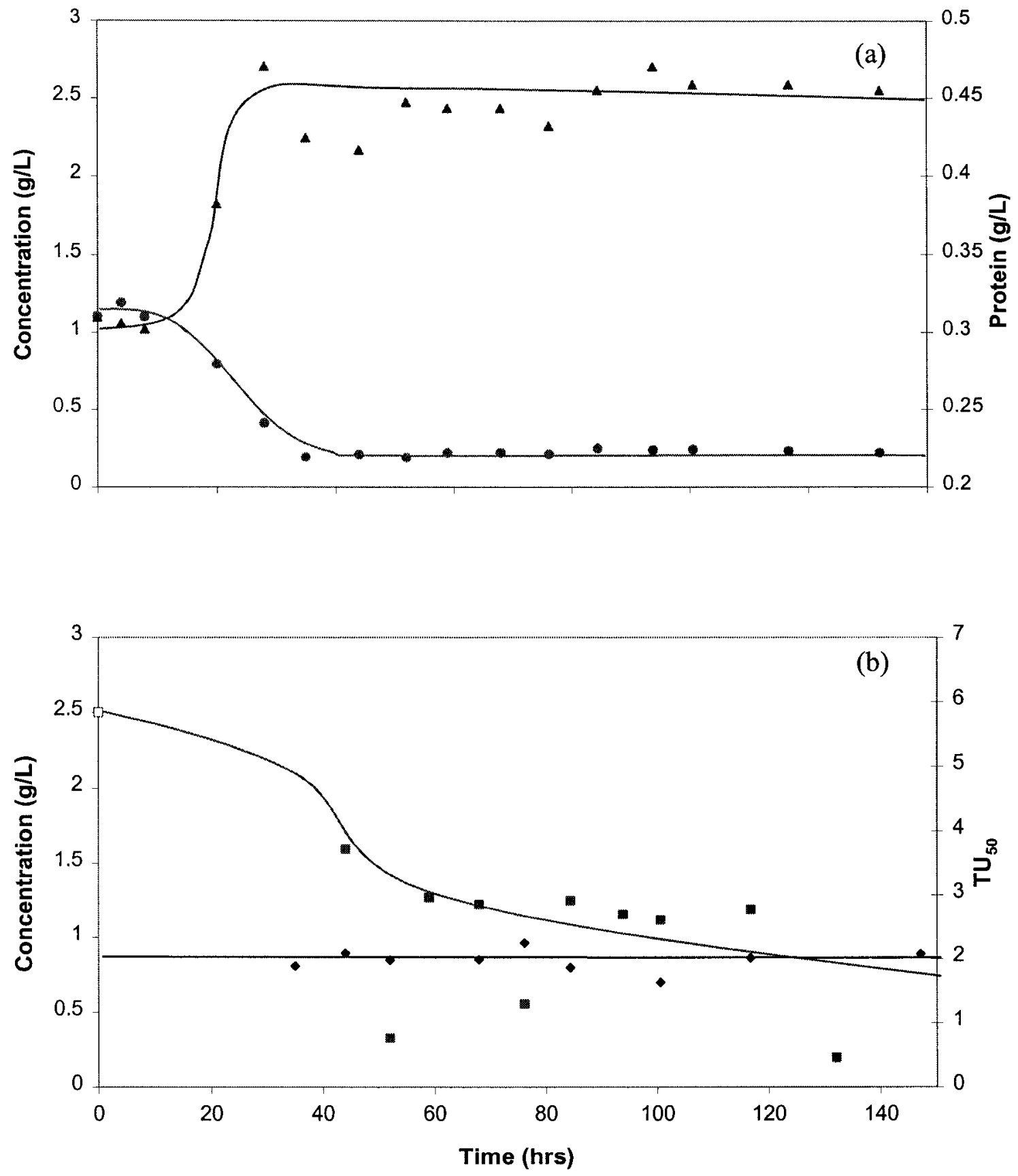

Figure 3-13. For a system using $R$. rubra: (a) protein $(\mathbf{A})$ and glucose concentration ( ) (b) concentration of dioctyl terephthalate (D) and toxicity ( ). The initial amount of plasticizer added to the reactor is also shown ( $\square$ ). 


\subsubsection{Characterization of Metabolites}

The metabolites appeared in the GC traces after samples of the broth had been extracted with chloroform and then injected into the GC. In order to determine if these metabolites were the toxic agents responsible for the increase in toxicity of the fermentation medium, it was first necessary to show that the extracted organic phase contained the toxic agents. An experiment was carried out that compared the toxicity of the aqueous and organic phase of three samples taken from the fermentation of D(PG)DB. The results for this test are shown in Figure 3-14. As the graph demonstrates, almost all of the toxicity in the original samples was accounted for in the organic phase. This indicated that the toxic agents were easily extracted from the broth and, thus, could be found in the extracts used for the GC.

The positions of the metabolite peaks observed in the GC traces are reported in Table 3-2. It can be seen that these peaks are different from the original plasticizers. The pattern of metabolites seen in the $\mathrm{D}(\mathrm{PG}) \mathrm{DB}$ system appeared as four different peaks (Figure 3-15) and metabolite seen in the D(EG)DB systems appeared as a single peak (Figure 3-16).

There are three main candidates from the breakdown of the $\mathrm{D}(\mathrm{PG}) \mathrm{DB}$ that were likely to match the pattern of metabolites observed in the GC of this system. These are benzoic acid, di-propylene glycol, and di-propylene glycol monobenzoate (monoester). The first two compounds were commercially available whereas the monoester had to be synthesized (Appendix I). From Table 3-2 it can be seen that the peak for benzoic acid and the three peaks for di-propylene glycol were different from the four peaks of the metabolite. The crude products obtained from two of the synthesis reactions, a trans 


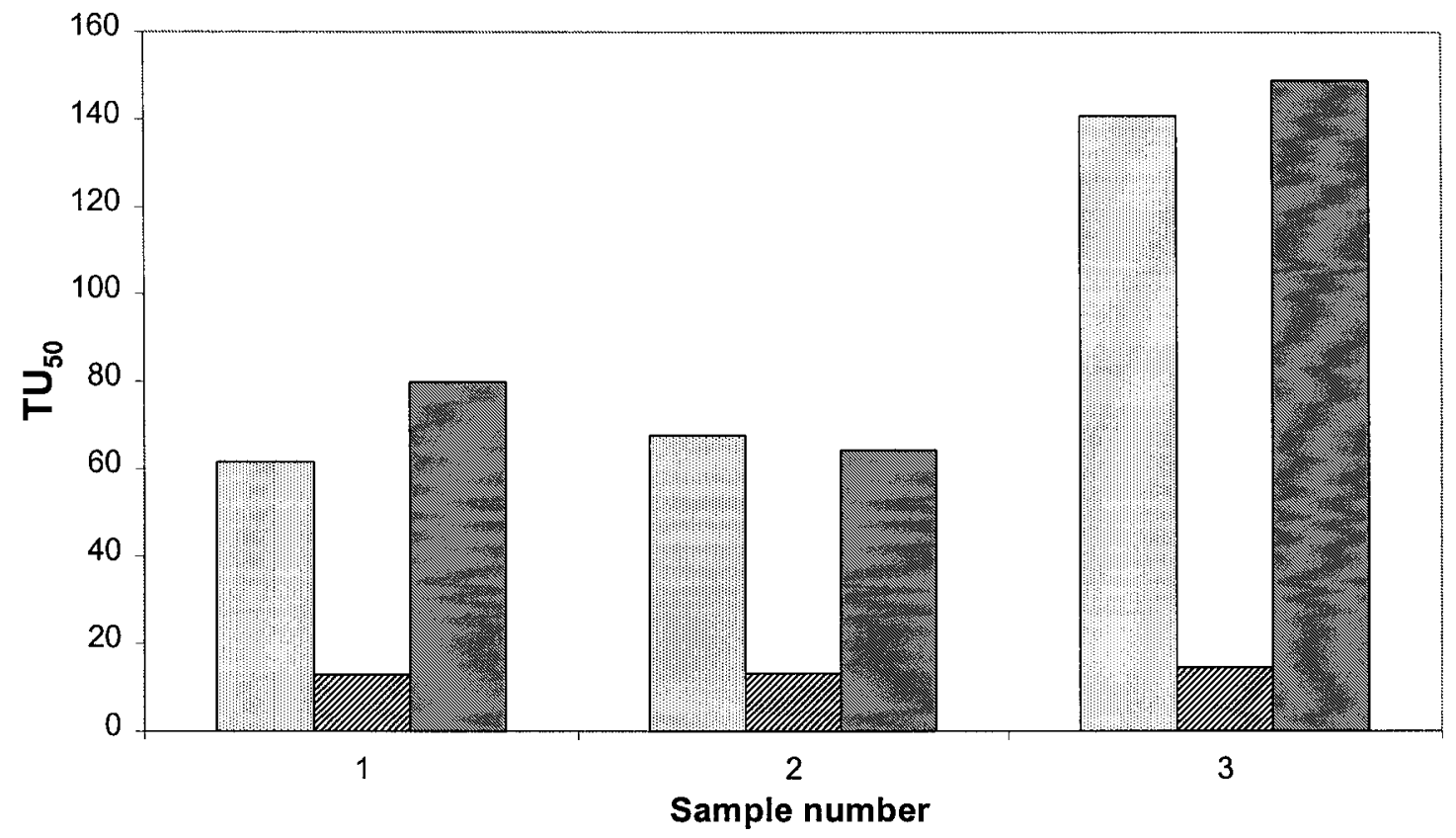

Figure 3-14. Toxicity comparison of the organic extract $(\mathbb{S})$ and aqueous raffinate $(\square)$ obtained from original samples $(\because)$ taken from the fermentation of $\mathrm{D}(\mathrm{PG}) \mathrm{DB}$. 
Table 3-2. Gas chromatograph-mass spectrophotometer (GC-MS) data for metabolites, plasticizers and other data.

\begin{tabular}{|c|c|c|}
\hline \multicolumn{2}{|c|}{ Compounds } & GC-MS Retention \\
\hline \multirow{4}{*}{$\begin{array}{l}\text { Pattern of Metabolites } \\
\text { observed in D(PG)DB } \\
\text { fermentation }\end{array}$} & a. & 13.52 \\
\hline & b. & 13.72 \\
\hline & c. & 13.75 \\
\hline & d. & 13.93 \\
\hline \multicolumn{2}{|c|}{ Metabolite observed in D(EG)DB fermentation } & 13.21 \\
\hline \multicolumn{2}{|c|}{ Pentadecane } & 10.8 \\
\hline \multirow{4}{*}{$\begin{array}{c}\text { Family of peaks } \\
\text { observed from trans } \\
\text { esterification reaction }\end{array}$} & a. & 13.50 \\
\hline & b. & 13.64 \\
\hline & c. & 13.77 \\
\hline & d. & 13.95 \\
\hline \multirow{4}{*}{$\begin{array}{c}\text { Family of peaks } \\
\text { observed from ester } \\
\text { synthesis reaction }\end{array}$} & a. & 13.53 \\
\hline & b. & 13.69 \\
\hline & c. & 13.80 \\
\hline & d. & 13.93 \\
\hline \multicolumn{2}{|c|}{ Benzoic acid } & 6.56 \\
\hline \multirow{3}{*}{ Di-propylene glycol } & a. & 4.75 \\
\hline & b. & 5.05 \\
\hline & c. & 5.33 \\
\hline \multicolumn{2}{|c|}{ Di-ethylene glycol dibenzoate } & 20.56 \\
\hline \multirow{3}{*}{$\begin{array}{c}\text { Di-propylene glycol } \\
\text { dibenzoate }\end{array}$} & a. & 20.36 \\
\hline & b. & 20.46 \\
\hline & c. & 20.68 \\
\hline
\end{tabular}




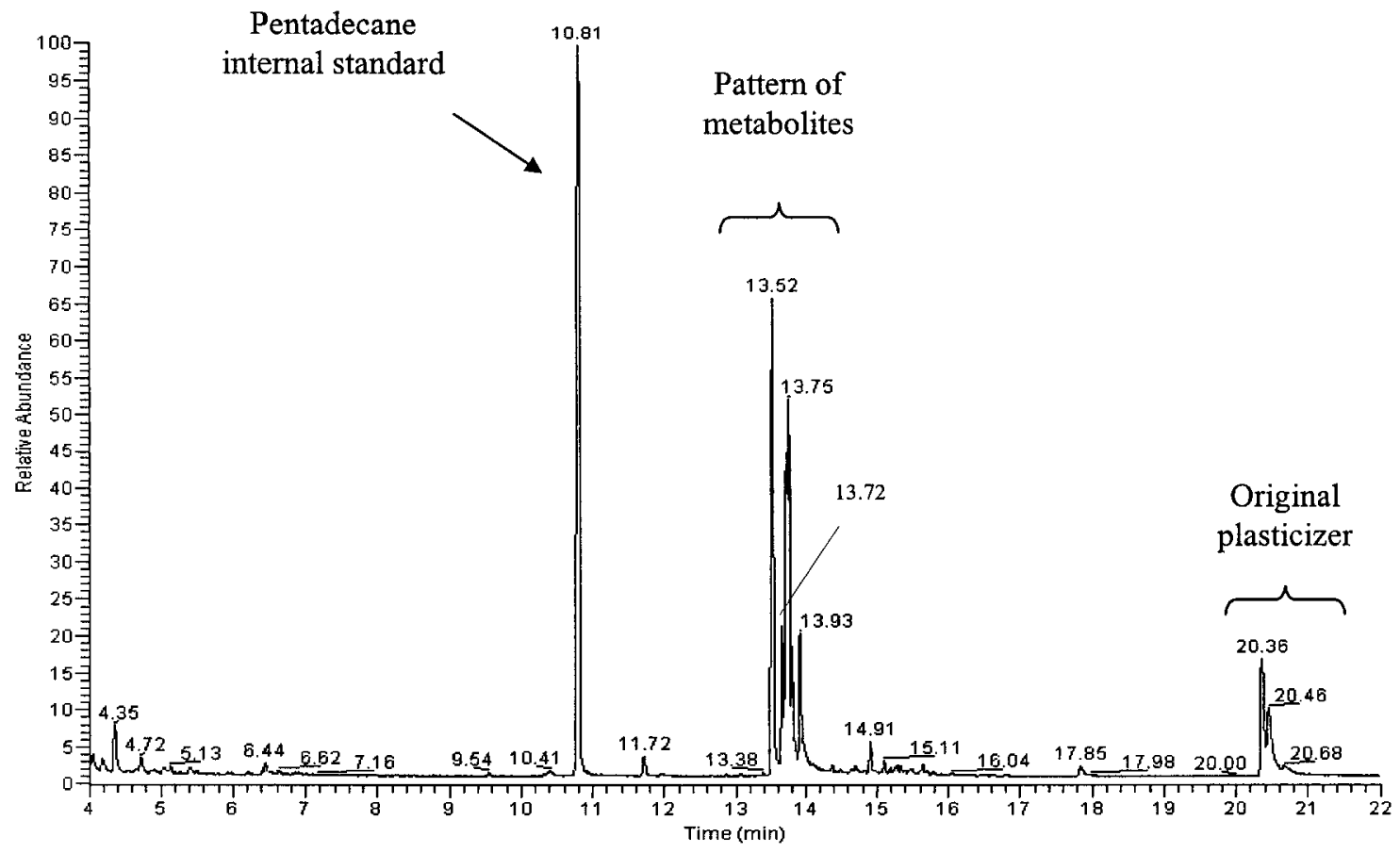

Figure 3-15. Gas chromatograph of a di-propylene glycol dibenzoate reactor sample.

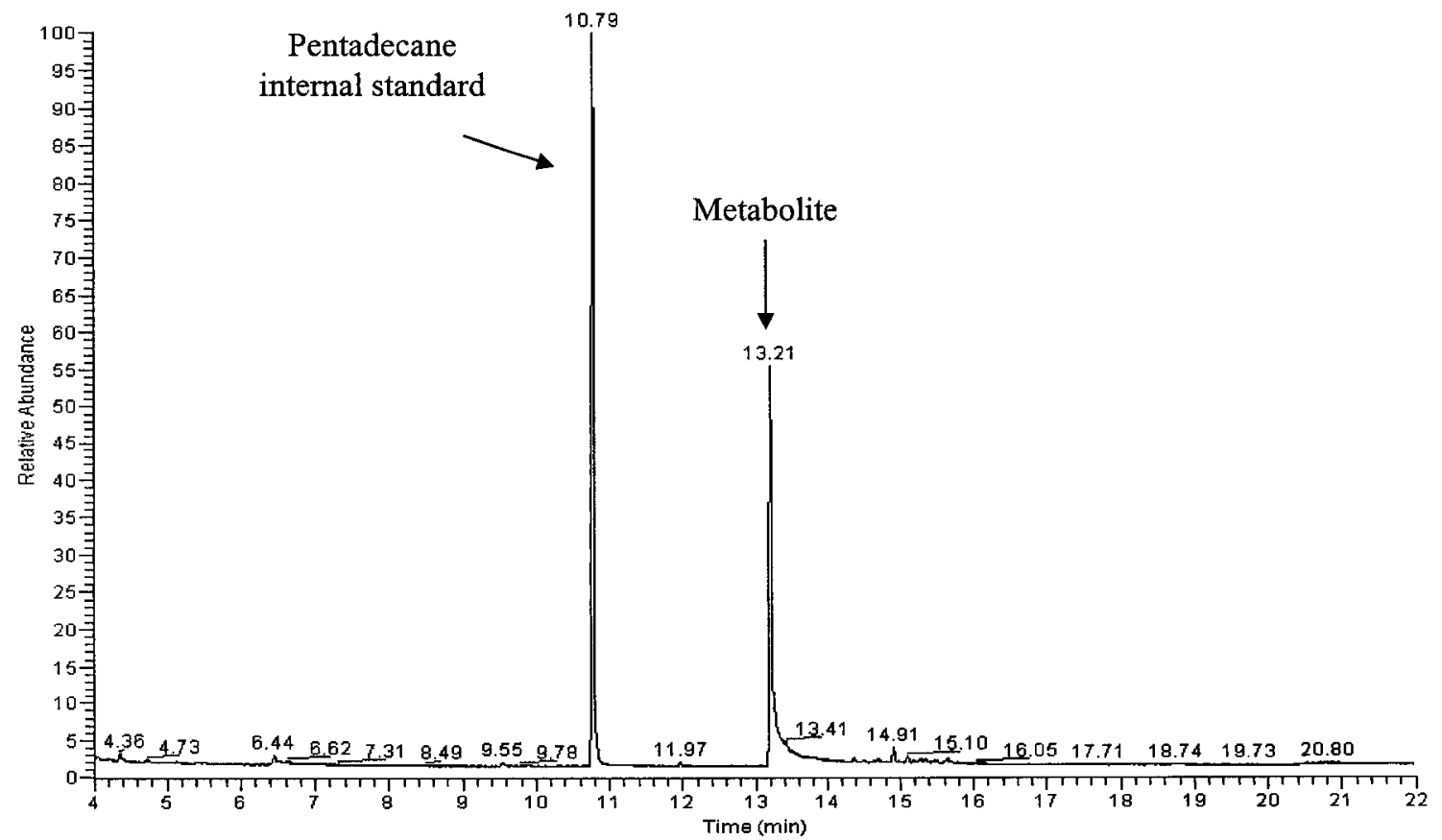

Figure 3-16. Gas chromatograph of a di-ethylene glycol dibenzoate reactor sample. 
esterification (Figure 3-17) and an ester synthesis (Figure 3-18), showed the same family of four peaks in their GC spectrums that were observed as the pattern of metabolites from the fermentation. In fact, the peak positions of the two reaction products and the pattern of metabolites in the growth studies matched up almost exactly. This seemed to suggest that the pattern of metabolites were isomers of a single compound; that is di-propylene glycol monobenzoate.

There are several peaks for this compound because, as shown in Figure 3-19, four possible isomer configurations exist for the monoester depending on the placement of the two methyl branches within the compound. It is reasonable to assume the same proportion of each isomer will be present at any given time. Thus, families of peaks were observed in the GC spectra for compounds for which this isomerization was possible.

Gas chromatography-mass spectroscopy (GC-MS) was later used to confirm that the monoester was the same compound as the metabolite. The parent ions for the family of four peaks from the two reaction products and the metabolite were identified from their respective MS fragmentation patterns (Figures 3-20, 3-21, 3-22). It was found that the parent ions were all the same and all very close (within the error of the GC-MS) to the molecular weight of di-propylene glycol monobenzoate (238 Daltons). The breakdown of the monoester was also found to be consistent with the peaks observed in the MS spectrum. For example, a phenyl ion has a molecular weight of 77 , benzoate has a molecular weight of 122 and propyl-benzoate 164 . All of these compounds make up pieces of the monoester and all of these peaks were seen in the MS fragmentation pattern for the metabolite and the synthesized monoesters. Based on these observations, there was little doubt that the metabolite was di-propylene glycol monobenzoate. 


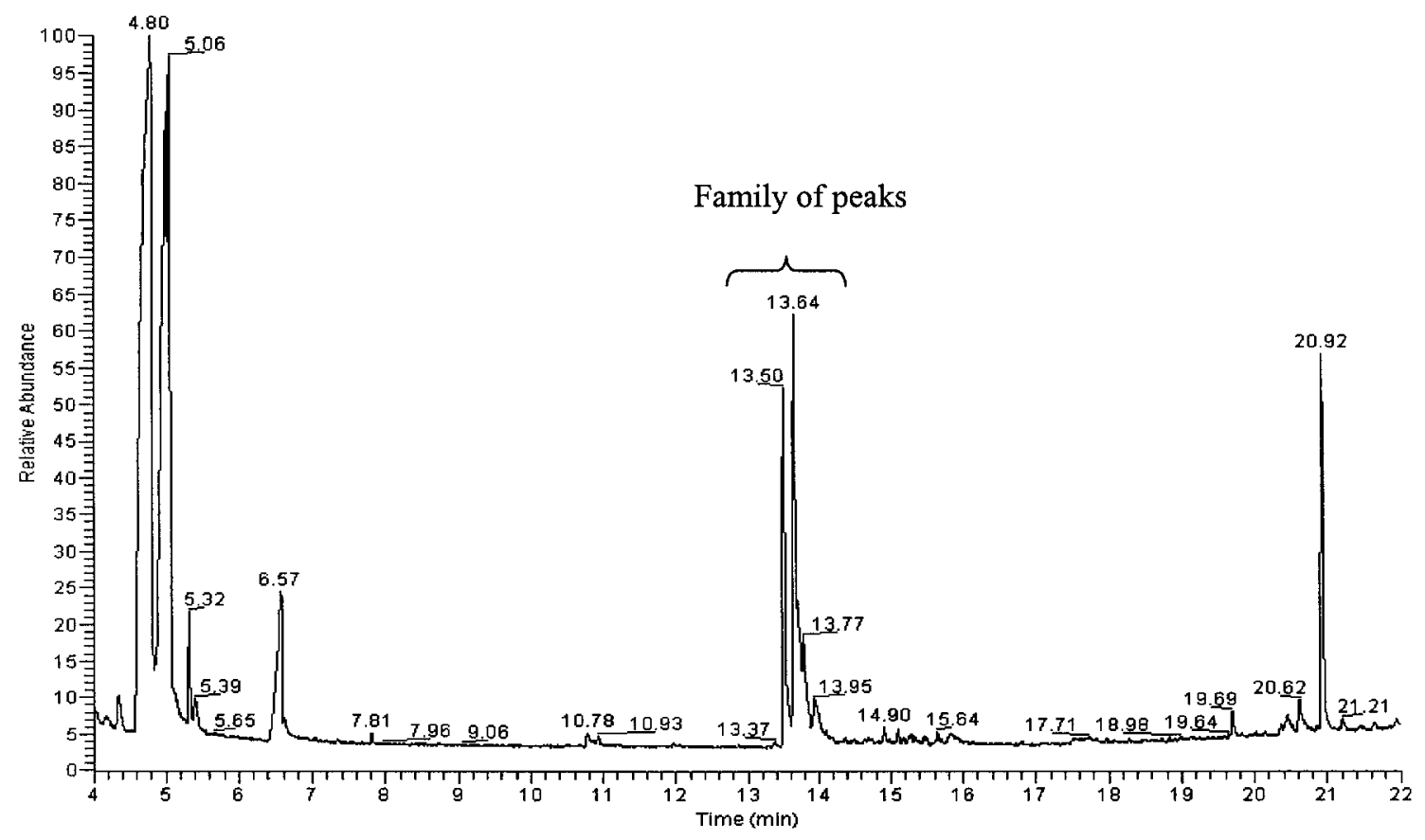

Figure 3-17. Gas chromatograph of the crude product obtained from a trans esterification reaction.

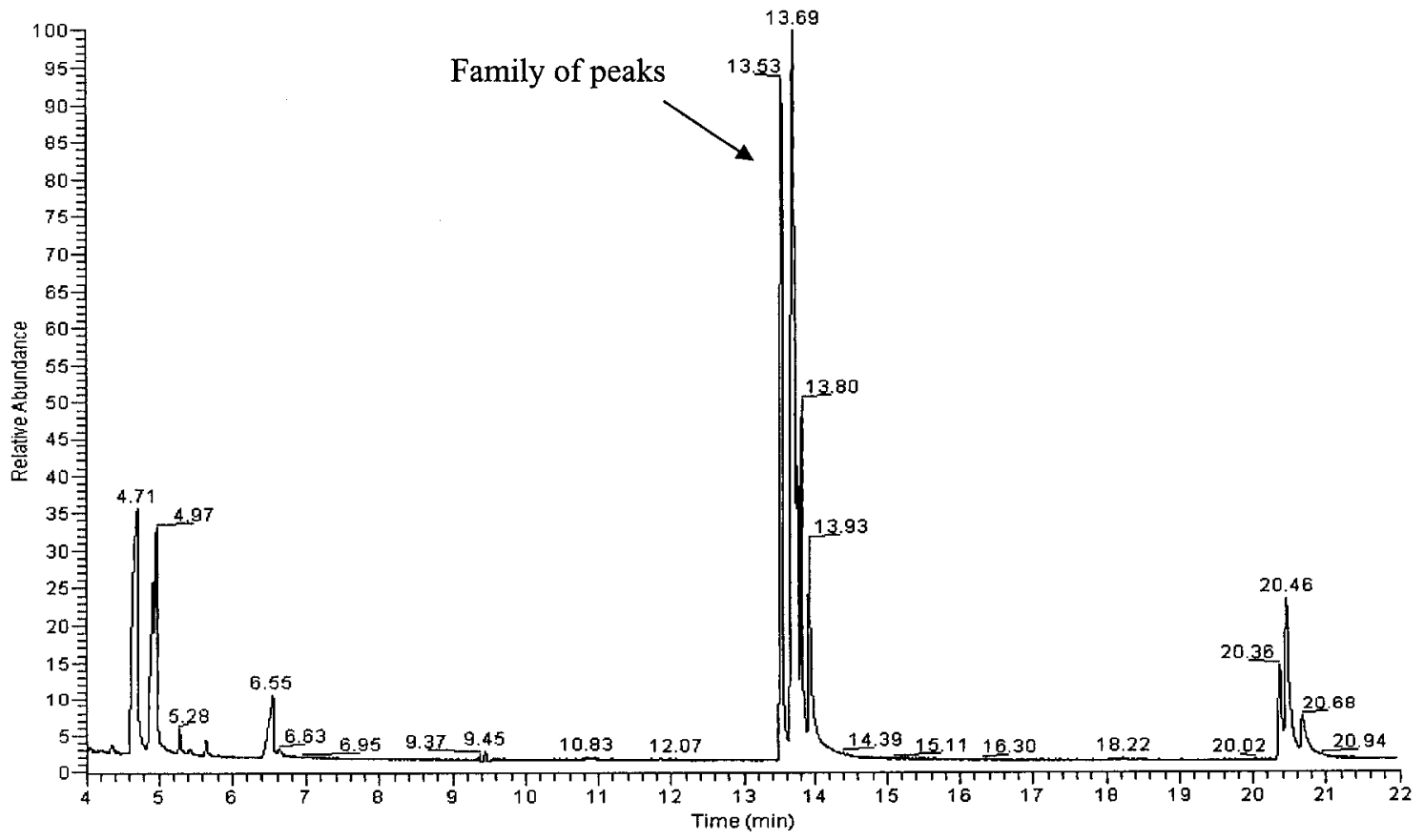

Figure 3-18. Gas chromatograph of the crude product obtained from an ester synthesis reaction. 
<smiles>CC(CO)OCC(C)OC(=O)c1ccccc1</smiles>

Isomer \#1<smiles>CC(CO)OCC(C)OC(=O)c1ccccc1</smiles>

Isomer \#3<smiles>CC(CO)OCC(C)OC(=O)c1ccccc1</smiles><smiles>CC(CO)OCC(C)OC(=O)c1ccccc1</smiles>

Figure 3-19. Isomer configurations of di-propylene glycol monobenzoate 


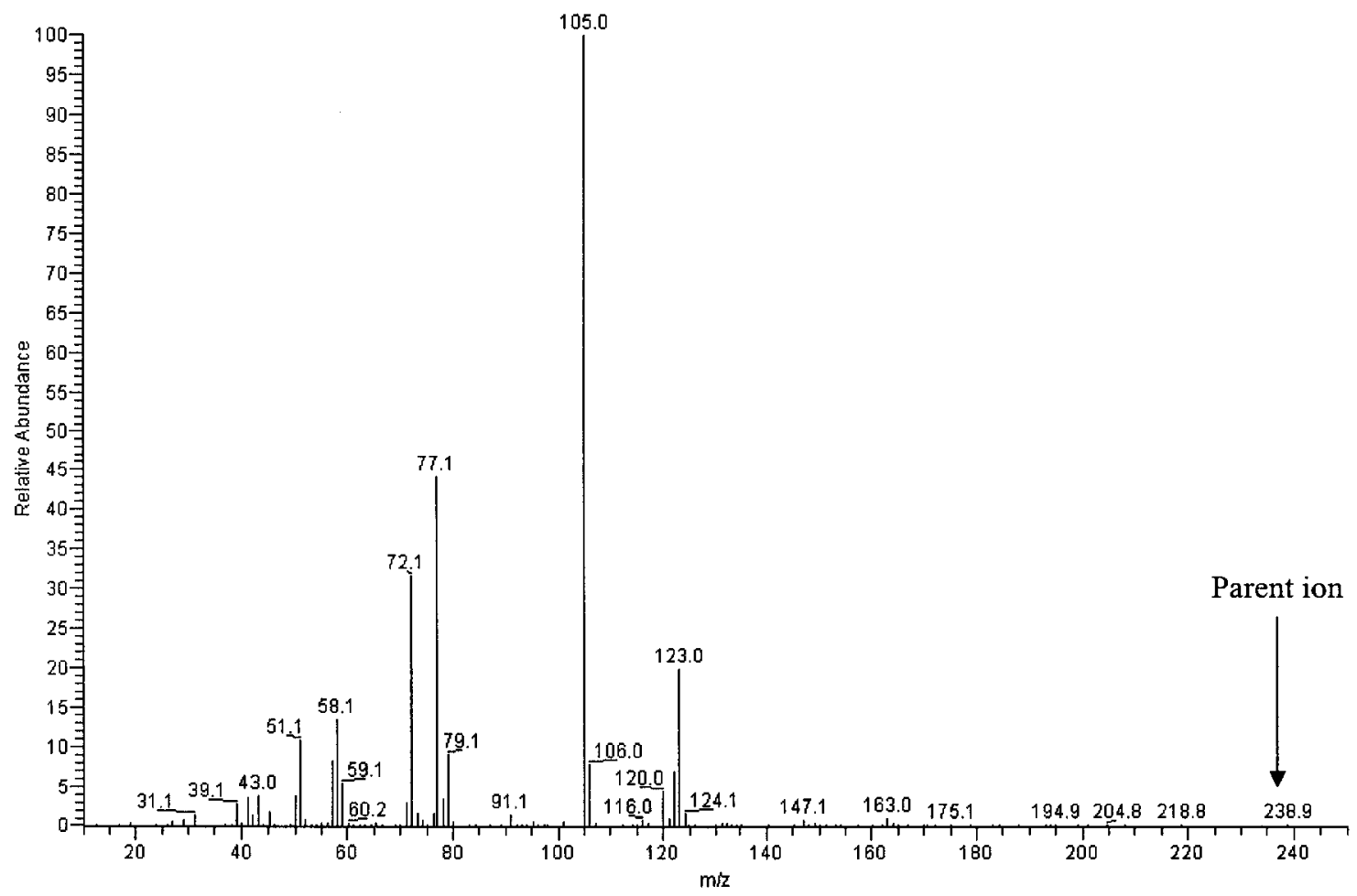

Figure 3-20. Mass spectrum fragmentation pattern of the pattern of metabolites observed in a di-propylene glycol dibenzoate reactor sample. 


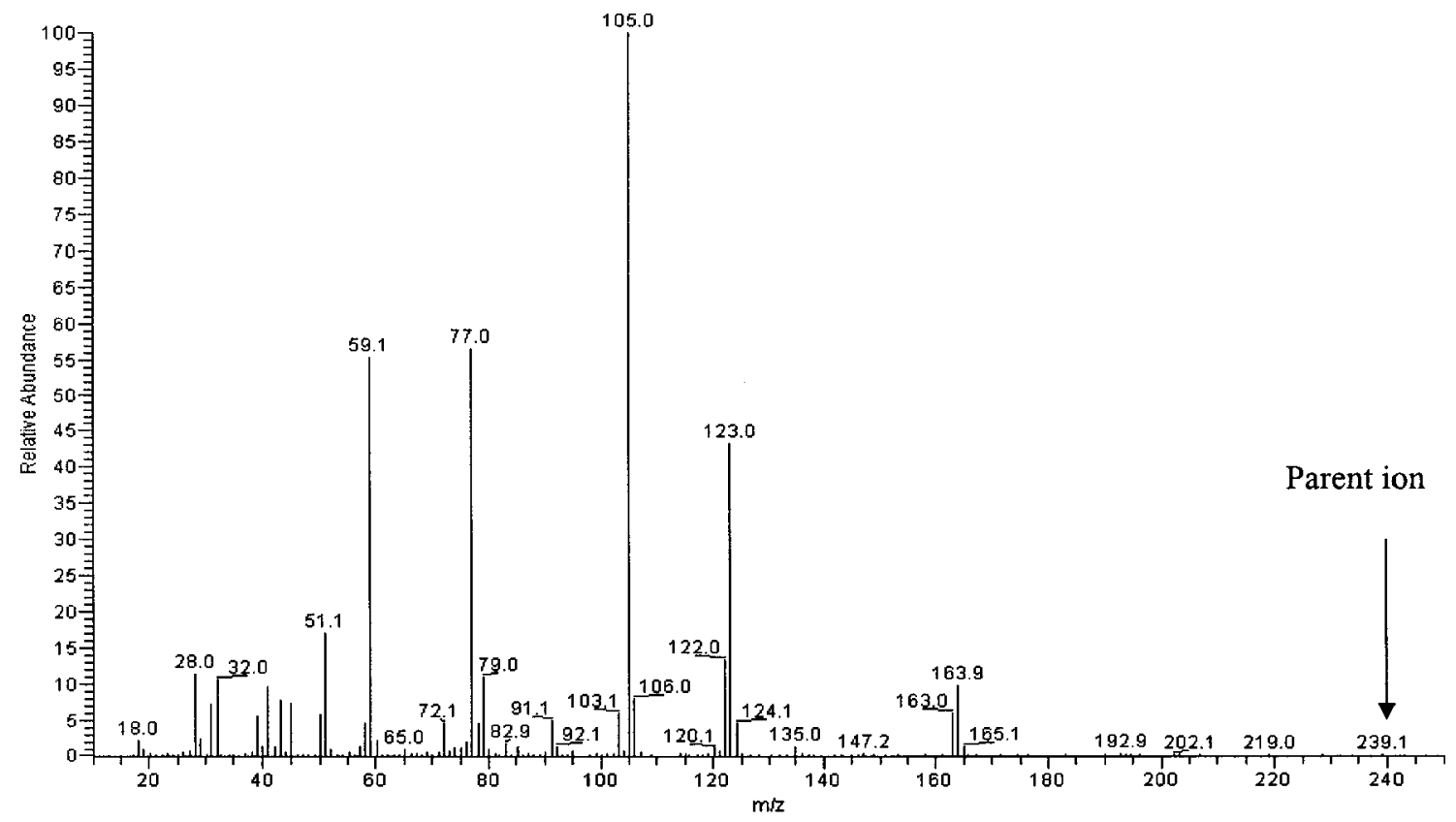

Figure 3-21. Mass spectrum fragmentation pattern of the family of peaks observed in the crude product from a trans esterification reaction.

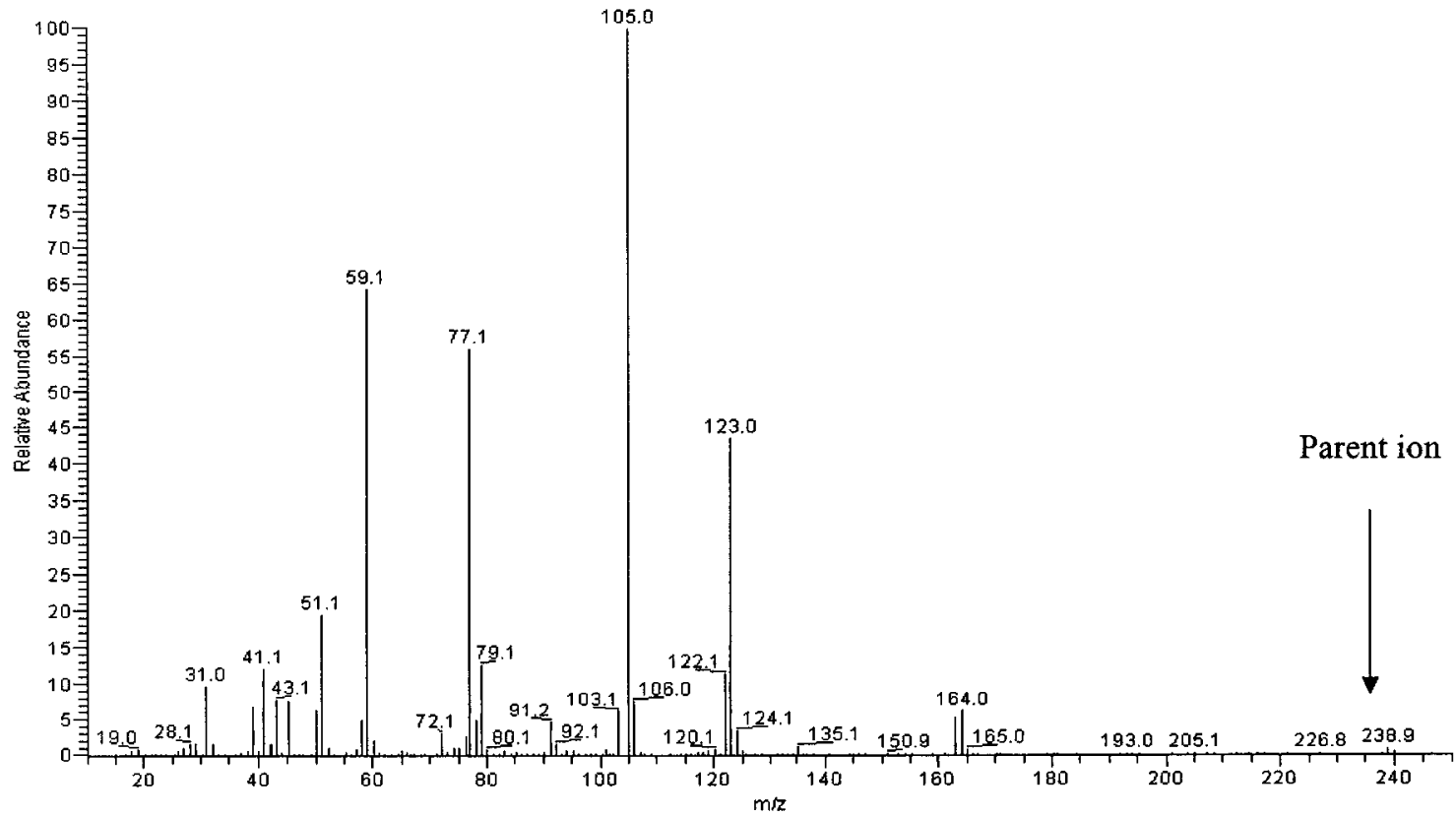

Figure 3-22. Mass spectrum fragmentation pattern of the family of peaks observed in the crude product from an ester synthesis reaction. 
The metabolite observed from the degradation of $\mathrm{D}(\mathrm{EG}) \mathrm{DB}$ was tentatively identified as di-ethylene glycol monobenzoate. This monoester has only a single possible isomer and, therefore, a single peak in the gas chromatograph spectrum. This peak was very close to the positions corresponding to the family of peaks for di-propylene glycol monobenzoate. It is reasonable to expect the peak positions to be relatively close for diethylene glycol monobenzoate and di-propylene glycol monobenzoate as both compounds are structurally similar. Although di-ethylene glycol monobenzoate was not synthesized, the MS fragmentation pattern of the metabolite peak (Figure 3-23) demonstrates that the parent ion of this compound was extremely close to the molecular weight of di-ethylene glycol monobenzoate (210 Daltons). The fragmentation pattern of the breakdown of this compound was also found to be consistent with the peaks observed in the MS spectrum. 


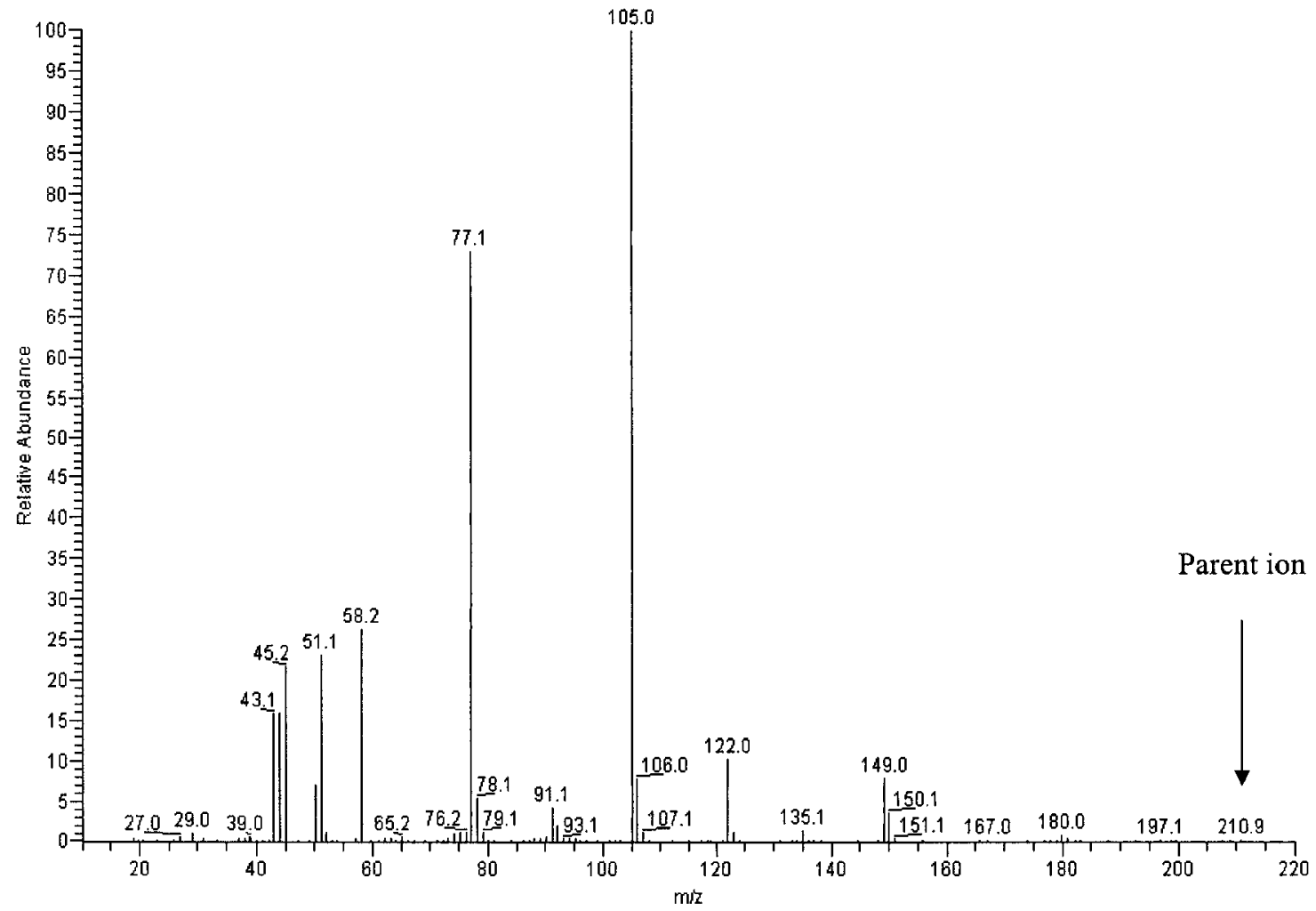

Figure 3-23. Mass spectrum fragmentation pattern of the single peak observed in a di-ethylene glycol dibenzoate reactor sample. 


\subsection{Toxicity of Pure Compounds}

Table 3-3 lists the toxicities of all of the relevant compounds involved in this work as measured by the Microtox assay. Each compound was added to an aqueous solution of MMSM and the concentrations of the different pure compounds were selected to be close to those observed throughout the course of the growth studies. It was found that the Microtox organism was very sensitive to di-propylene glycol monobenzoate, which had a toxicity of $110 \mathrm{TU}_{50}$ at a concentration of $1.0 \mathrm{~g} / \mathrm{L}$. This corresponds to an $\mathrm{EC}_{50}$ of $0.038 \mathrm{mmol} / \mathrm{L}$, which, molecule per molecule is 6 times more toxic than phenol, which was used as a standard and control for this assay. Benzoic acid had a relatively low toxicity and $\mathrm{D}(\mathrm{PG})$ had a negligible toxicity. All of the plasticizers involved in this research had considerably low toxicities except for the two dibenzoate plasticizers whose $\mathrm{EC}_{50}$ values (on a molar basis) were comparable to the $\mathrm{EC}_{50}$ of phenol. 
Table 3-3. Toxicity of compounds in MMSM ${ }^{1}$.

\begin{tabular}{|c|c|c|c|}
\hline Compounds & $\begin{array}{c}\text { Concentration of } \\
\text { Compound (g/L) }\end{array}$ & $\begin{array}{c}\text { Toxicity, } \\
\left.\text { (as TU }_{50}\right)\end{array}$ & $\begin{array}{c}\text { Toxicity, } \\
\left(\text { as EC }_{50}, \mathbf{m m o l} / \mathrm{L}\right)\end{array}$ \\
\hline $\begin{array}{c}\text { Di-propylene glycol } \\
\text { monobenzoate }\end{array}$ & 0.5 & 55.2 & 0.038 \\
\hline Di-propylene glycol & 0.5 & Below detection & - \\
\hline Phenol & 0.5 & 22.5 & 0.236 \\
\hline Benzoic acid & 0.5 & 2.7 & 1.97 \\
\hline $\begin{array}{c}\text { Di-propylene glycol } \\
\text { dibenzoate }\end{array}$ & 2.5 & 24.9 & 0.294 \\
\hline $\begin{array}{c}\text { Di-ethylene glycol } \\
\text { dibenzoate }\end{array}$ & 2.5 & 15.0 & 0.531 \\
\hline \begin{tabular}{c} 
Dioctyl phthalate \\
\hline $\begin{array}{c}\text { Dioctyl } \\
\text { terephthalate }\end{array}$
\end{tabular} & 2.5 & Below detection & - \\
\hline $\begin{array}{c}\text { Bis (2-ethyhexyl) } \\
\text { adipate }\end{array}$ & 2.5 & Below detection & - \\
\hline
\end{tabular}

1. $\mathrm{MMSM}=$ minimum mineral salt medium as defined in materials and methods. 


\section{DISCUSSION}

\subsection{Biodegradation of Plasticizers by Eukaryotes}

\subsubsection{Preliminary Screening of Plasticizers}

The investigation of the breakdown of plasticizers in the presence of other, more easily utilized substrates has relevance to actual environmental problems. It is reasonable to assume that after plasticizers leach out of the plastics and into the environment, they would be in contact with a variety of microorganisms and many other potential substrates including water-soluble materials such as carbohydrates. This is a particularly realistic model for the environment around waste disposal sites.

In the preliminary screening, attempts were made to degrade selected plasticizers using several eukaryotes. Either hexadecane or glucose was used as a co-substrate in all of these experiments. Previous work on the degradation of these plasticizers showed that many microorganisms could not sustain growth on plasticizers as the sole carbon source $^{48}$. Other workers have speculated that a co-substrate promotes metabolism of plasticizers $^{44}$. Thus, as the organisms metabolize the primary substrate, enzymes are produced which are able to break down bonds in the plasticizers without any energy or biomass being produced. In general, all of the plasticizers considered in this research were at least partially degraded by at least some of the organisms, if another substrate was present.

Lang and Stanhope suggested that dibenzoate plasticizers are easier to degrade than more commonly used plasticizers: bis (2-ethylhexyl) adipate (B(EH)A), dioctyl phthalate (DOP) and dioctyl terephthalate (DOTP) ${ }^{32}$. The results obtained from the preliminary studies support this conclusion (Table 3-1). Both dibenzoates were 
significantly degraded by all of the organisms except for C. bombicola for which they were still moderately degraded. It has also been suggested that as well as being easily degraded, the dibenzoates have low toxicities ${ }^{32,49}$. If true, these observations would make them ideal alternatives for the three more commonly used plasticizers.

The results from previous work done on the degradation of the adipate and two phthalate plasticizers were surprising because they demonstrated that instead of a reduction in toxicity, a significant increase was observed as growth in the presence of these compounds proceeded ${ }^{44}$. This was linked to the appearance of several metabolites from the partial degradation of the plasticizers. Since the dibenzoate plasticizers appear to be more easily degraded than those studied in this earlier work, it was anticipated that their degradation might result in complete degradation and no toxic intermediates.

Rhodotorula rubra was selected for a detailed growth study to test this speculation since it was able to significantly degrade both dibenzoates using glucose as a co-substrate and because it has been shown to be able to grow on plastic materials ${ }^{48}$.

\subsubsection{Degradation of Dibenzoate}

In all of the growth studies with $R$. rubra, significant amounts of glucose were present even at the end of the experiment. These fermentations were continued for several days and lasted well into the stationary phase. For the growth of Rhodotorula rubra, either glucose was not the rate limiting substrate or the presence of the dibenzoate plasticizers inhibited the growth of the organism to a certain extent. Inhibition seems to be the less likely explanation since the amount of biomass observed with the plasticizer 
was consistent with the amount observed in the control experiment when only glucose was present (Figure 3-3).

Degradation of di-propylene glycol dibenzoate $(\mathrm{D}(\mathrm{PG}) \mathrm{DB})$ commenced in the exponential phase of growth and continued throughout the experiment (Figure 3-4 (a)). Approximately $80 \%$ of the plasticizer was degraded after 130 hours of the fermentation. These results are consistent with the work of Lang and Stanhope ${ }^{32}$, but because of the report of the accumulation of toxic metabolites arising from the biodegradation of some plasticizers ${ }^{44}$, it was important to look for intermediates resulting from partial degradation. In fact, as the concentration of plasticizer decreased, several new peaks appeared in the gas chromatographs of the extracts of the broth (Figure 3-15). These peaks were determined to be isomers of di-propylene glycol monobenzoate.

Figure 4-1 is a possible degradation mechanism that is consistent with the metabolite being the monoester. Rhodotorula rubra has been shown to produce esterase enzyme $^{48}$ which could hydrolyze one of the ester bonds of $\mathrm{D}(\mathrm{PG}) \mathrm{DB}$. This would release di-propylene glycol monobenzoate as well as benzoic acid. It is reasonable to conclude that the benzoic acid was metabolized further since there was no significant accumulation of this compound in the fermentation medium. Hydrolysis of the second ester bond of $\mathrm{D}(\mathrm{PG}) \mathrm{DB}$ would yield di-propylene glycol but this must be a slow step for this organism as there was a substantial amount of the monoester present at the end of every experiment. As well, di-propylene glycol was not observed at any time in any of the experiments. 
<smiles>CCOC(=O)OCCOCCOCCOCC(C)OCC(C)OC(=O)c1ccccc1</smiles>
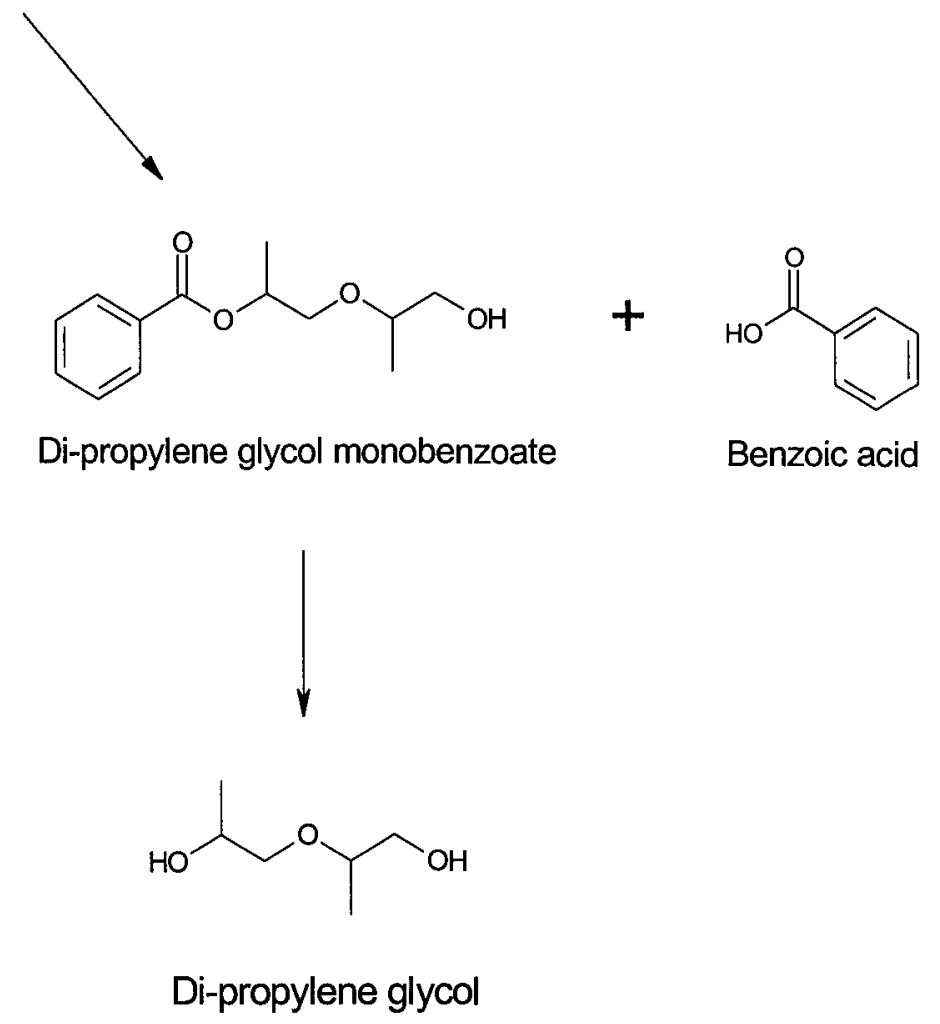

Figure 4-1. Proposed mechanism for the production of di-propylene glycol monobenzoate from the degradation of $\mathrm{D}(\mathrm{PG}) \mathrm{DB}$. 
The pattern of degradation of di-ethylene glycol dibenzoate (D(EG)DB) by $R$. rubra was found to be very similar to that observed of di-propylene glycol dibenzoate but, the former was more readily hydrolyzed as is evident from its disappearance from the medium after 140 hours of the fermentation (Figure 3-7 (a)). However, the proposed monoester metabolite (di-ethylene glycol monobenzoate) was observed to be very difficult to degrade and it accumulated in the fermentation medium (Figure 3-7 (b)). The concentration of the metabolite began to level off as the concentration of $\mathrm{D}(\mathrm{EG}) \mathrm{DB}$ approached zero as would be expected for a product resulting from the breakdown of the plasticizer.

When Aspergillus niger was used to degrade $\mathrm{D}(\mathrm{EG}) \mathrm{DB}$, extensive degradation of the plasticizer occurred within 130 hours (Figure 3-9 (a)). The peak, attributed to diethylene glycol monobenzoate, was observed to increase and then quickly decrease within a relatively narrow 40-hour period as the concentration of the plasticizer was continuously decreasing (Figure 3-9 (b)). The amount of metabolite was found to be an order of magnitude smaller when $A$. niger was used to degrade the plasticizer than when R. rubra was used.

D(EG)DB was the more easily degraded of the two plasticizers and Aspergillus niger was more efficient at breaking down both $\mathrm{D}(\mathrm{EG}) \mathrm{DB}$ and the metabolites than $R$. rubra. However, it is important to note that there was at least a small amount of monoester remaining at the end of the all of the growth studies with this type of plasticizer. 


\subsubsection{Degradation of B(EH)A, DOP, and DOTP Plasticizers}

The degradation of bis (2-ethylhexyl) adipate (B(EH)A), dioctyl phthalate (DOP) and dioctyl terephthalate (DOTP) was also tested using R. rubra. These are examples of commonly used plasticizers that could be replaced by the dibenzoates if they were to prove to be less toxic. All three plasticizers were degraded individually using glucose as a co-substrate. With $\mathrm{B}(\mathrm{EH}) \mathrm{A}$ or DOP, approximately $80 \%$ and $90 \%$ percent of the plasticizers were degraded respectively. The results observed with DOP are consistent with the previous work done using this strain of $R . r u b r a^{47}$. The organism seemed to be able to use the plasticizers as a carbon source in both cases since the amount of biomass nearly doubled as compared with the degradation of the dibenzoates (Figures 3-11 (a) and 3-12 (a)). The growth of the organism was also somewhat faster than that with the dibenzoates, achieving the stationary phase within 30 hours of the fermentation as opposed to 40 hours. Only $70 \%$ of DOTP was degraded during fermentation. Although the stationary phase was reached in 40 hours, the amount of biomass in the system was still only comparable to the amount seen when the organism was degrading the dibenzoates (Figure 3-13 (a)).

The results obtained with these $\mathrm{B}(\mathrm{EH}) \mathrm{A}, \mathrm{DOP}$ and DOTP were unexpected given that Lang and Stanhope had suggested the dibenzoate plasticizers were easier to biodegrade $^{32}$. The rates of degradation for all five plasticizers were comparable. In fact, R. rubra actually grew fastest and produced the most biomass when $\mathrm{B}(\mathrm{EH}) \mathrm{A}$ or DOP were present.

However, the most important difference observed for all three of these plasticizers, relative to the dibenzoates, was that no metabolites were detected. This was 
also found to differ from the work reported by $\mathrm{Nalli}^{44}$. In his studies using the bacterium Rhodococcus rhodochrous, all three of $\mathrm{B}(\mathrm{EH}) \mathrm{A}, \mathrm{DOP}$, and DOTP resulted in at least one intermediate being present in the broth at the end of the fermentation.

\subsection{Toxicity Studies}

The Microtox assay relates toxicity to a decrease in luminescence by Vibrio fischeri, which is proportional to the degree of inhibition caused by the compound being tested. The biochemical pathways that are affected by the toxic agents have been shown to be similar to those of higher organisms ${ }^{11,22}$. Other commonly used toxicity tests have

also been correlated with the Microtox assay ${ }^{12,34,51}$. Therefore, the toxicity data obtained with the Microtox were considered to be representative of toxic responses observed in the environment.

Usually, the degradation of plasticizers using microorganisms is considered a method of bioremediation. Logically, the biodegradation of potentially harmful compounds in the environment would result in an overall decrease in toxicity of a contaminated site. This was not true for the microorganism studied here. For the degradation of each dibenzoate plasticizer with $R$. rubra, the initial toxicities of the nondegraded plasticizers in the broth were determined to be an order of magnitude higher than those observed in the control fermentation, where the organism was grown solely on glucose. This indicated that $\mathrm{D}(\mathrm{PG}) \mathrm{DB}$ and $\mathrm{D}(\mathrm{EG}) \mathrm{DB}$ had some initial toxicity (Table 33). As the organism began to grow and degrade the dibenzoates, the expected decrease in toxicity was not observed. In fact, Figures 3-5, 3-6 and 3-8, demonstrate that the toxicity 
of the fermentation broth increased dramatically in every case. The control experiment without any plasticizer present demonstrated no toxicity at any time (Figure 3-3).

The increase in toxicity was intimately connected to the increase of the monoester metabolites. For example, in Figure 3-6, the concentration of the monoester peaked at $1.0 \mathrm{~g} / \mathrm{L}$ at the same time as the peak in toxicity at a $\mathrm{TU}_{50}$ of around 140 was observed. (This was the highest toxicity observed for the batch fermentation of any plasticizer in this research). Moreover, when the concentration of the monoester decreased, the toxicity appeared to follow the same trend.

Based on these observations, it was reasonable to assume that the measured toxicity of the pure monoester would account for a large part of the observed toxicity in this system. According to Table 3-3, the toxicity of di-propylene glycol monobenzoate at its maximum observed concentration in the medium $(\sim 1.0 \mathrm{~g} / \mathrm{L})$, corresponded to a $\mathrm{TU}_{50}$ of 110 . This is very close to the observed $\mathrm{TU}_{50}$ of 140 , which reinforces the conclusion that the monoester was responsible for the majority of the toxicity in the broth. Further evidence of this was shown in Figure 3-14. When the organic compounds (including the monoester) were extracted from cell-free broth samples of the reactor, almost all of the toxicity was removed. Only a small fraction of the toxicity of the broth was accounted for in the aqueous phase.

Given that the monoester did not account for all of the toxicity in the reactor broth, there must have been other factors that influenced the toxicity. The additional toxicity might have been caused by the presence of small amounts of either benzoic acid or di-propylene glycol (two compounds appearing in the proposed degradation mechanism of $\mathrm{D}(\mathrm{PG}) \mathrm{DB}$ ) but, from Table 3-3 it can be seen this was not the case. 
Benzoic acid has a very low toxicity and the toxicity of $\mathrm{D}(\mathrm{PG})$ was not detectable. It would seem more reasonable to assume that the non-degraded plasticizer accounted for the remainder of the toxicity since it did have some initial toxicity. However, based on the concentration of the plasticizer at the maximum observed toxicity $(\sim 0.75 \mathrm{~g} / \mathrm{L})$, only an additional $\mathrm{TU}_{50}$ of 7.5 would have contributed to the toxicity demonstrated by the monoester.

There might have been other components in the broth that contributed to the observed toxicity of the monoester but it was not possible to isolate all of the aqueous and organic components in the samples from the fermentation broth and test each of their individual toxicities.

The proposed monoester metabolite (di-ethylene glycol monobenzoate) was not synthesized. However, this compound is structurally very similar to the monoester that was characterized. There was a single peak in the appropriate region of the GC that increased in concentration during the fermentation experiments and this compound should only have one isomer. It is reasonable to assume that this compound would be toxic because the very similar di-propylene glycol monobenzoate was so toxic. Therefore, the increase in toxicity of the system shown in Figure 3-8 could be attributed to the build-up of di-ethylene glycol monobenzoate in the medium. The patterns of toxicity and metabolite with time showed, once again, how intimately connected the metabolite was with toxicity. The maximum observed toxicity occurred at a $\mathrm{TU}_{50}$ of about 70 . Though this was only half the toxicity observed from the fermentation of D(PG)DB with $R$. rubra, the system was still considered to be fairly toxic. 
When $A$. niger was used to degrade $\mathrm{D}(\mathrm{EG}) \mathrm{DB}$, a much lower toxicity was observed than with $R$. rubra. The toxicity peaked at a $\mathrm{TU}_{50}$ of approximately 25 . The reduced toxicity was attributed to there being an order of magnitude less of the proposed monoester metabolite in the fermentation medium. This system was still considered to be fairly toxic since the final toxicity was comparable to that of the phenol standard.

For the degradation of each of $\mathrm{B}(\mathrm{EH}) \mathrm{A}, \mathrm{DOP}$ and DOTP with $R$. rubra, there was no significant increase in toxicity of the broth throughout the fermentation of any of these plasticizers. This was attributed to there being no evidence of any metabolite production in every case. However, there was a small increase followed by a decrease in toxicity observed at the beginning of the fermentation of each of $\mathrm{B}(\mathrm{EH}) \mathrm{A}$ and $\mathrm{DOP}$ as shown in Figures 3-11 (b) and 3-12 (b). Although the toxicities of these two systems were considered to be low, the observed trends indicated that some slightly toxic metabolites might have been produced early in the fermentation of each of these plasticizers that were not observed in the GC spectrum of the extracted samples of the broth. Either these metabolites were quickly degraded after they appeared in the medium or they may have not been extracted from the broth and were present in the aqueous phase.

The results of the present study were found to differ from the results of previous work done on the degradation of these same three plasticizers using bacteria, where the production of 2-ethyl hexanol from $\mathrm{B}(\mathrm{EH}) \mathrm{A}$ and 2-ethyl hexanoic acid from $\mathrm{B}(\mathrm{EH}) \mathrm{A}$, DOP, and DOTP were determined to be responsible for an increase in toxicity in all three systems $^{44}$. In order for these metabolites to be produced, it was evident from the degradation mechanisms proposed by Nalli for B(EH)A, DOP, and DOTP with Rhodococcus rhodochrous, that the bacteria would have to first produce a different 
monoester compound from each plasticizer when one of the ester bonds from the original plasticizer was hydrolyzed. However, Nalli reported no evidence of any accumulation of these monoesters in his work. This leads to the speculation that the bacteria were able to further hydrolyze the second ester bond of these compounds, which resulted in even more of the end metabolites being produced.

From the growth experiments with the two dibenzoate plasticizers in which there was a definite accumulation of metabolites in the media, it was concluded that $R$. rubra was unable to further degrade the monoester metabolites that were produced. It was evident that the kinetics of the first hydrolysis step was much faster than the kinetics of the second hydrolysis step for these compounds. This differed from experiments with $\mathrm{B}(\mathrm{EH}) \mathrm{A}, \mathrm{DOP}$, or DOTP where the esterase enzyme produced by this microorganism was able to hydrolyze both ester bonds of each of the starting materials.

\subsection{Potential Impact}

Plasticizers can be introduced into the environment after leaching out of plastics and migrating through existing water transport systems. While in the environment, it is likely that these compounds would be readily available for microbial interaction and subsequent biodegradation. Initially, it was reasonable to assume that the introduction of these plasticizers into the environment would not present a serious problem due to their low toxicities and eventual degradation. However, the results of recent work ${ }^{44}$ using common soil bacteria has shown that the biodegradation of three of the more commonly used plasticizers in industry, including an adipate and two phthalate plasticizers, resulted in a marked increase in the toxicity of the solution. This increase in toxicity was 
attributed to the production of metabolites from the incomplete degradation of these plasticizers.

Based on these observations, it was apparent that other plasticizers should be considered as replacements for the more commonly used plasticizers. Recent work has suggested that dibenzoate plasticizers, due to their low toxicity and relative ease of biodegradation, could be the ideal alternatives for the plasticizers now used in children's toys $^{32}$ and in other PVC applications ${ }^{49}$. However, the work presented here shows that the degradation of these alternative compounds could lead to the accumulation of metabolites that are more toxic than the currently used plasticizers or their metabolites.

Evidently, the problem associated with the acute toxicity of metabolites from the incomplete breakdown of plasticizers is more widespread than anticipated. It seems that this will remain so until safer alternatives are developed or new methods of remediation are implemented. 


\section{CONCLUSION}

All of the six plasticizers studied in this research were at least partially degraded by at least some of the common soil fungi and yeasts investigated. However, this degradation only occurred in the presence of another, more easily utilized substrate.

Two plasticizers, di-propylene glycol dibenzoate $(\mathrm{D}(\mathrm{PG}) \mathrm{DB})$ and di-ethylene glycol dibenzoate $(\mathrm{D}(\mathrm{EG}) \mathrm{DB})$, have been proposed as safer alternatives to more commonly used plasticizers including bis 2-ethylhexyl adipate $(\mathrm{B}(\mathrm{EH}) \mathrm{A})$, dioctyl phthalate (DOP) and terephthalate (DOTP). In the present study, it was observed that these alternative plasticizers were slightly more easily degraded than the commonly used plasticizers but the degree of mineralization was found to be less. Degradation of $\mathrm{D}(\mathrm{PG}) \mathrm{DB}$ and $\mathrm{D}(\mathrm{EG}) \mathrm{DB}$ resulted in significant amounts of intermediates and these intermediates were shown to be significantly more toxic that the original starting materials.

A mechanism was proposed for the production of the metabolite resulting from the degradation of $\mathrm{D}(\mathrm{PG}) \mathrm{DB}$. This metabolite, identified as a monoester (di-propylene glycol monobenzoate), was formed by the hydrolysis of a single ester bond in the original plasticizer. It was confirmed that this compound was responsible for the acute toxicity observed in the media as $R$. rubra grew in the presence of the plasticizer.

The metabolite observed when $\mathrm{D}(\mathrm{EG}) \mathrm{DB}$ was being degraded by $R$. rubra was not isolated but it was tentatively identified as the monoester di-ethylene glycol monobenzoate by analogy to the $\mathrm{D}(\mathrm{PG}) \mathrm{DB}$ system. The same metabolite was observed when $\mathrm{D}(\mathrm{EG}) \mathrm{DB}$ was degraded by the fungus, Aspergillus niger. 
Although $\mathrm{B}(\mathrm{EH}) \mathrm{A}$, DOP, and DOTP were degraded by $R$. rubra, this degradation did not result in the production of significant amounts of metabolites or increases in the toxicity of the media. This contrasts with earlier work in which it was reported that all three of these plasticizers were degraded by bacteria and resulted in the accumulation of significant amounts of toxic metabolites.

Because of concerns about environmental impact, dibenzoates have been proposed as alternatives for the plasticizers now being used. However, this work has shown that careful consideration must be given to the interaction of the dibenzoates with commonly encountered microorganisms. In the examples shown here, the initial hydrolysis does formally remove the dibenzoates from the media but the resulting monoester was very toxic and resistant to further biodegradation. Therefore, the dibenzoates may not necessarily be a satisfactory alternative to commonly used plasticizers. 


\section{REFERENCES}

1. Albro P. W., Chapin R. E., Corbett J. T., Schroeder J. and Phelps J. (1989) Mono2-ethylhexyl phthalate, a metabolite of di-(2-ethylhexyl) phthalate causally linked to testicular atrophy in rats. Toxicology and Applied Pharmacology, 100:193200.

2. Arendt, William D. (1981) Performance of dipropylene glycol dibenzoate in plasticizer blends for vinyl plastisols. Velsicol Chem. Corp., Chicago, IL, USA. Plast. Compd., 4(4), 33-4, 38, 40-2, 44.

3. Arendt, William D. and Barrington, David W. (1997) Mono and dibenzoate ester blends as caulk plasticizers that are bioresistant to fungal growth. PCT Int. Appl., $37 \mathrm{pp}$.

4. Arendt, William. (1979) Replacing DOP as a plasticizer for polyvinyl chloride. Velsicol Chem. Corp., Ann Arbor, MI, USA. Plast. Eng., 35(9), 46-9.

5. Arendt, William. (1981) Using glycol benzoates in acrylic latex caulks. Velsicol Chem. Corp., Chicago, IL, USA. Adhes. Age, 24(11), 56-60.

6. Augustin, Jozef and Muncnerova. (1994) Degradation pathways of aromatic hydrocarbons in fungi and bacteria. Biologia (Bratislava), 49(3), 289-9.

7. Bailey, James E. and Ollis, David F. (1986) Biochemical Engineering Fundamentals, $2^{\text {nd }}$ ed. Published by McGraw-Hill, Inc., USA, 140-3.

8. Barber E. D., Fox J. A. and Giordano C. J. (1994) Hydrolysis, absorption and metabolism of di(2-ethylhexyl) terephthalate in the rat. Xenobiotica, 24(5):441450 .

9. Begley, Timothy H. and Hollifield, Henry C. (1990) Migration of dibenzoate plasticizers and polyethylene terephthalate cyclic oligomers from microwave susceptor packaging into food-simulating liquids and food. J. Food Prot., 53(12), 1062-6.

10. Bohnert, T., Izadi, R., Pitman, S. and Stanhope, B. (1998) Recent developments: benzoate esters in polyvinyl resilient flooring. Velsicol Chem. Corp., Northbrook, IL, USA. Annu. Tech. Conf. - Soc. Plast. Eng, 56th(Vol. 3), 32843290.

11. Bulich A. A. and Bailey G. (1995) Environmental toxicity assessment using luminescent bacteria. In Environmental Toxicology Assessment. Taylor and Francis Inc., Bristol, Pennsylvania.:29-40. 
12. Bulich A. A., Greene M. W. and Isenberg D. L. (1981) Reliability of the bacterial luminescence assay for the determination of the toxicity of pure compounds and complex effluents. Aquatic Toxicology and Hazard Assessment: Fourth Conference. ASTM STP 737. American Society for Testing and Materials:338347.

13. Castle L., Mercer, A. J. and Gibert J. (1988) Migration from plasticized films into foods. 4. Use of polymeric plasticizers and lower levels of di-(2-ethylhexyl) adipate plasticizer in PVC films to reduce migration into foods. Food Additives and Contaminants, 5, 277-282.

14. Choudhry S., Lox F. and Decroly P. (1994) The migration of plastic components: the quantification of influence from mechanical stresses and vibration. Packaging Technology and Science, 7:163-167.

15. Colon I., Caro D., Bourdony, C. J. and Rosario O. (2000) Identification of phthalate esters in serum of young Puerto Rican girls with premature breast development. Environmental Health Perspectives, 108 (9):895-900.

16. Eaton R. W. and Ribbons D. W. (1982) Metabolism of dibutylphthalate and phthalate by Micrococcus sp. Strain 12B. Journal of Bacteriology, 151(1):48-57.

17. Edenbaum, J. (1992) Plastic Additives and Modifiers Handbook, Published by Van Nostrand Reinhold, New York, New York.

18. Faouzi M.A., Dine T., Luyckx M., Gressier B., Goudaliez F., Mallevais M.L., Brunet C., Cazin M. and Cazin J.C. (1994) Leaching of diethylhexyl phthalate from PVC bags into intravenous teniposide solution. International Journal of Pharmaceutics, 105:89-93.

19. Foss K; Clues to the early puberty mystery. Globe and Mail (Canada) Tuesday, November 14, 2000. R5.

20. Gibbons J. A. and Alexander M. (1989) Microbial degradation of sparingly soluble organic chemicals: Phthalate esters. Environmental Toxicology and Chemistry, 8:283-291.

21. Graham P. R.(1973) Phthalate ester plasticizer-Why and how they are used. Environmental Health Perspectives, 3:3-12.

22. Hinwood A. L., McCormick M. J. and McCormick R. J. (1988) Evaluation of the Microtox technique for assessment of toxicity in water and wastewaters. A report prepared for the Environmental Protection Authority of Victoria.

23. Illinois EPA, Endocrine Disruptors Strategy, Feb. 1997 
24. Jayabalan M. and Lizymol P. P. (1995) Studies on the migration behaviour of chemically treated plasticizer poly(vinyl chloride) for blood contact applications. Journal of Materials Science Letters, 14:589-591.

25. Jobling,S. Reynolds,T. White,R. Parker,M.G. and Sumpter J.P.(1995) A variety of environmentally persistent chemicals, including some phthalate plasticizers, are weakly estrogenic, Environmental Health Perspectives, 103(6):582-587

26. Katayama-Hirayama, K., Tobita, S. and Hirayama, K. (1992) Aromatic degradation in yeast Rhodotorula rubra. Water Sci. Technol., 26, 773-81.

27. Kates, Morris. (1972) Techniques of lipidology: isolation, analysis and identification of lipids. Published by North-Holland Publishing Company, Amsterdam.

28. Keith Y., Cornu M. C., Canning P. M., Foster J., Lhuguenot J. C. and Elcombe C. R. (1992) Peroxisome proliferation due to di (2-ethylhexyl) adipate, 2ethylhexanol and 2-ethylhexanoic acid. Archives of. Toxicology, 66:321-326.

29. Keyser P., Pujar B. G., Eaton R. W. and Ribbons W. R. (1976) Biodegradation of the phthalates and their esters by bacteria. Environmental Health Perspectives, 18:159-166.

30. Koch A. L. (1994) Growth Measurement. Methods for general and molecular bacteriology. American Society for Microbiology, Washington, D.C. 248-277.

31. Kyrides, L. P. (1933). U.S. Pat. 1,923,938.

32. Lang, Jiamin and Stanhope, Bruce E. (2001) Benzoate plasticizer for flexible PVC injection moulded toy applications. Velsicol Chem. Corp., Northbrook, IL, USA. Plast. Addit. Compd., 3(6), 30-33.

33. Lau O-W and Wong S-K (1997) Mathematical model for the migration of plasticizers from food contact material into solid food. Analytica Chimica Acta, 347:249-256.

34. Lebsack M. E., Anderson A. D., DeGraeve G. M. and Bergman H. L. (1981) Comparison of bacterial luminescence and fish bioassay results for fossil-fuel process waters and phenolic constitutes. Aquatic Toxicology and Hazard Assessment: Fourth Conference. ASTM STP 737. American Society for Testing and Materials:348-356.

35. Lhuguenot J-C, Mitchell A. M., Milner G., Lock E. A. and Elcombe C. R. (1985) The metabolism of di(2-ethylhexyl) phthalate (DEHP) and mono-(2-ethylhexyl) phthalate (MEHP) in rats: In vivo and in vitro dose and time dependency of metabolism. Toxicology and Applied Pharmacology, 80:11-22. 
36. Lindley, N. D., Pedley, Joanna F., Kay, S. P. and Heydeman, M. T. (1986) The metabolism of yeasts and filamentous fungi which degrade hydrocarbon fuels. Int. Biodeterior., 22(4), 281-7.

37. Loftus N.J., Laird W. J. D., Steel G.T. and Woollen B.H. (1993) Metabolism and pharmacokinetics of deuterium-labeled di-2-(ethylhexyl) adipate (DEHA) in humans. Food and Chemical Toxicology, 31(9):609-614.

38. Lunberg P., Hogberg J., Garberg P., Lundberg I., Dobson S. and Howe P. (1992). Diethylhexyl phthalate. Report 131. World Health Organization, Geneva, Switzerland.

39. Mayer F. L. and Sanders H. O. (1973) Toxicity of phthalic acid ester in aquatic organisms. Environmental Health Perspectives, 3:153-157.

40. Microbics Corporation. (1994) Microtox M500 Manual: A toxicity testing handbook, Microbics Corp., Carlsbad, CA.

41. Milligan, S. R., Balasubramanian, A. V. and Kalita, J. C.(1998) Relative potency of xenobiotic estrogens in an acute in vivo mammalian assay. Environmental Health Perspectives, 106 (1):23-26.

42. Mitchell A. M., Lhuguenot J-C, Bridges J. W. and Elcombe C. R. (1985) Identification of the proximate peroxisome proliferator(s) derived from di(2ethylhexyl) phthalate. Toxicology and Applied Pharmacology, 80:23-32.

43. Mozisek, Max. (1974) Evaluation of the diffusion processes of diethyleneglycol dibenzoate in poly(vinyl chloride). Res. Inst. SIGMA, Olomouc, Czech. Gummi, asbest, Kunstst., 27(6), 450-461.

44. Nalli, S. (2001) Biodegradation of Plasticizers: characterization and toxicity of their metabolites. Masters Thesis, Dept. of Chem. Eng, McGill University, Montreal, Canada.

45. Osmon, J. L., Klausmeier, R. E. and Jamison, E. I. (1970) Ability of selected yeast cultures to degrade plasticized polyvinyl systems. Dev. Ind. Microbiol., 11, 447-52.

46. Papaspyrides C. D. and Tingas S. G. (1998) Comparison of isopropanol and isooctane as food stimulants in plasticizer migration tests. Food Additives and Contaminants, 15(6):681-689.

47. Peciulyte, D. (1997) Degradation of dioctylphthalate ester by yeasts Rhodotorula rubra J-96-1 and microfungus Aspergillus puniceus J-86-2. Institute of Botany, Vilnius, Lithuania. Biologija, (2), 29-32. 
48. Peciulyte, D. (1997) Utilization of phthalates esters by microorganisms. Institute of Botany, Vilnius, Lithuania. Biologija, (2), 33-37.

49. Peng, Xianyu and Zhang, Xuequn. (2000) Application of a new plasticizer diethylene glycol dibenzoate (DEDB) in poly(vinyl chloride) products. Beijing University of Chemical Technology, Beijing, Peop. Rep. China. Zhongguo Suliao, 14(8), 64-67.

50. Pitman, Stephen, Stanhope, Bruce, Arendt, William and Bohnert, Tom. (1999) New developments in benzoate esters for the resilient flooring industry. Velsicol Chem. Ltd., Basingstoke, UK. PVC 99, [PVC Brighton Conf.], 21 ${ }^{\text {st }}, 239-248$. Publisher: IOM Communications Ltd., London, UK.

51. Qureshi, A. A., Colemanand R. N. and Paran J. N. (1983) Evaluation and refinement of the Microtox test for the use in toxicity screening. Toxicity Screening Procedures Using Bacteria Systems. Toxicology Series Vol I. B. J. Dutka and D. Liu. New York, Marcel Dekkar 1:89-118.

52. Romero, M. C., Gatti, E. M., Cazau, M. C. and Arambarri, A. M. (1998) Isolation and characterization of yeast hydrocarbon degraders. Instituto Spegazzini, Facultad Ciencias Naturales Museo, Universidad Nacional La Plata, La Plata, Argent. UFZ-Ber., (18), 64-70.

53. Rosenberg M., Barki M., Bar-Ness R., Goldberg S., Doyel R. J. (1991) Microbial adhesion o hydrocarbons (MATH). Biofouling, 4(1-3):121-128.

54. Sears, J. Kern and Darby, Joseph R. (1982) The Technology of Plasticizers, Published by John Wiley \& Sons, New York.

55. Seibert, Walter, Neumann, Uwe, Steuer, Mike and Cierniak, Karl-heinz. (2001) Floor- and wallcoverings with good resistance to plasticizer migration. Dunlop Tech Gmbh, Germany, Eur. Pat. Appl., 6 pp.

56. Sharman M., Read W. A., Castle L. and Gilbert J. (1994) Levels of di-(2ethylhexyl) phthalate and total phthalate esters in milk, cream, butter and cheese. Food Additives and Contaminants, 11(3):375-385.

57. Sokolov, Yu. I., Davydov, E. R., Demanova, N. F. And Gololobov, A. D. (1981) Utilization of alkyl aromatic hydrocarbons by the yeast Candida guilliermondii. All-Union Res. Inst. Synth. Prot. Compd., Moscow, USSR. Prikl. Biokhim. Mikrobiol., 17(5), 660-8.

58. Stalling D. L., Hogan J. W. and Johnson J. L. (1973) Phthalate ester residuesTheir metabolism and analysis in fish. Environmental Health Perspectives, 3:159173. 
59. Sullivan K. F., Atlas E. L. and Giam C. S. (1982) Adsorption of phthalic acid ester from sea water. Environmental Science and Technology, 16:428-32.

60. Tepper L. B. (1973) Phthalic acid ester - An overview. Environmental Health Perspectives, 3:179-182.

61. Walker, J. D., Austin, H. F. and Colwell, R. R. (1975) Utilization of mixed hydrocarbon substrate by petroleum-degrading microorganisms. J. Gen. Appl. Microbiol., 21(1), 27-39. 


\section{APPENDIX}




\section{A.1. Synthesis Reactions for Di-Propylene Glycol Monobenzoate}

In order to confirm whether di-propylene glycol monobenzoate was the metabolite from the degradation of $\mathrm{D}(\mathrm{PG}) \mathrm{DB}$, it was first necessary to synthesize the compound. Four different reactions were attempted to synthesize the monoester.

The first was an ester hydrolysis reaction where $10.0 \mathrm{~mL}$ of the original plasticizer was heated $\left(\sim 60^{\circ} \mathrm{C}\right.$ for 15 minutes) in a $20.0 \mathrm{~mL}$ solution of basic water ( $\sim \mathrm{pH}$ 11) in an attempt to break one of the ester bonds of $\mathrm{D}(\mathrm{PG}) \mathrm{DB}$ to produce the monoester. There was evidence of some benzoic acid in the gas chromatogram of the product, which indicated that the reaction did occur to a small extent but, for the most part, the crude product consisted mainly of the original plasticizer and there was no evidence of the monoester. This failure was attributed mostly to the very low solubility of the plasticizer in water.

The second reaction was a biological analogue of this attempt at base hydrolysis. $5.0 \mathrm{mg}$ of esterase enzyme (from porcine or rabbit liver) was added to catalyze the breakage of the ester bond. The reaction was carried out in $50.0 \mathrm{~mL}$ of buffered water (pH 8) containing approximately $0.06 \mathrm{~mL}$ of $\mathrm{D}(\mathrm{PG}) \mathrm{DB}$. The contents of the reactor were heated to $30{ }^{\circ} \mathrm{C}$ for a period of four hours. Again, there was no evidence of the monoester in the gas chromatograph spectrum of the crude product. This time neither of the esterases used was soluble so there was insufficient contact of the catalyst with the plasticizer and aqueous phase. Also, enzymes are very sensitive to temperature changes ${ }^{7}$ and the esterases may have denatured at some point during the reaction, which would render them inactive. 
The third reaction was a trans-esterification, which proved to be more successful than the previous attempts. Approximately $0.15 \mathrm{~mL}$ of $\mathrm{D}(\mathrm{PG}) \mathrm{DB}$ was reacted with 100.0 $\mathrm{mL}$ of denatured alcohol ( $85 \%$ ethanol, $15 \%$ methanol) to obtain a crude product. The reaction was base catalyzed ( 1 pellet of $\mathrm{KOH}$ ) and the temperature in the reactor was maintained at $110^{\circ} \mathrm{C}$ for a period of 6 hours. The reaction time as well as the initial amount of plasticizer added had to be small enough to prevent the reaction from going to completion (where both ester bonds would have been broken to produce di-propylene glycol).

The GC spectrum of the crude product contained peaks for benzoic acid, dipropylene glycol $(\mathrm{D}(\mathrm{PG}))$ and the original plasticizer. The presence of the acid and $\mathrm{D}(\mathrm{PG})$ indicated that a reaction had occurred.

The fourth reaction was an ester synthesis reaction. The monoester was synthesized by reacting $10.0 \mathrm{~mL}$ of benzoyl chloride with $20.0 \mathrm{~mL}$ of di-propylene glycol at a temperature of $60^{\circ} \mathrm{C}$ for 6 hours. The reaction was carried out under nitrogen and acetone was used as a solvent. An excess of di-propylene glycol to benzoyl chloride was maintained in the reactor in order to prevent the reaction from going to completion and producing the original plasticizer, $\mathrm{D}(\mathrm{PG}) \mathrm{DB}$. The $\mathrm{GC}$ spectrum of the crude product showed that the monoester was produced in a much higher yield then observed in the trans-esterification synthesis and, therefore, this was the product used to obtain a large enough sample for characterization. 


\section{A.2. Isolation of Di-Propylene Glycol Monobenzoate}

Preparative thin layer chromatography (TLC) was used to separate the monoester from the other components in the crude product mixtures. The solvent system used for the analysis contained hexanes, ethyl ether and acetic acid in a 80:20:1 ratio, as reported by Kates for the separation of neutral lipids ${ }^{27}$. Table A-1 shows the $R_{F}$ values for the 5 bands that separated from the crude product of the trans-esterification reaction. Each band was collected, extracted with chloroform, and then analyzed with the gas chromatograph (GC). The GC spectrum of band \#3 from the TLC plate showed the appearance of a new pattern of peaks that matched almost exactly with the family of peaks that were observed as the metabolite from the fermentation. Preparative TLC of the crude product from the ester synthesis reaction resulted in the separation of 4 main bands (Table A-2). The GC spectrum for the extract of band \#1 showed only the peaks attributed to isomers of the monoester. 
Table A-1. $R_{F}$ values for bands separated from the crude product of the transesterification reaction.

\begin{tabular}{|c|c|}
\hline Bands & R $_{\text {Fanges }}$ \\
\hline$\# 1$ & 0.03 \\
\hline$\# 2$ & $0.1-0.13$ \\
\hline$\# 3$ & $0.34-0.38$ \\
\hline$\# 4$ & $0.4-0.44$ \\
\hline$\# 5$ & $0.59-0.68$ \\
\hline
\end{tabular}

Table A-2. $R_{F}$ values for bands separated from the crude product of the ester synthesis reaction.

\begin{tabular}{|c|c|}
\hline Bands & RF Ranges $^{\text {\#1 }}$ \\
\hline$\# 2$ & $0.03-0.13$ \\
\hline$\# 3$ & $0.15-0.23$ \\
\hline$\# 4$ & $0.28-0.35$ \\
\hline
\end{tabular}

